\title{
ARTICLE
}

\section{Exploring the Scope of the Isothiourea-mediated Synthesis of Dihydropyridinones}

Received ooth January 2012, Accepted ooth January 2012

DOI: $10.1039 / \times 0 x \times 00000 x$

www.rsc.org/

\author{
Pei-Pei Yeh, David S. B. Daniels, Charlene Fallan, Eoin Gould, Carmen Simal, \\ James E. Taylor, Alexandra M. Z. Slawin and Andrew D. Smith*
}

\section{Introduction}

The direct organocatalytic asymmetric functionalisation of readily available, bench-stable carboxylic acids ${ }^{1}$ towards valueadded products has received much attention since the seminal report by Romo and co-workers in 2001 on the intramolecular nucleophile-catalysed aldol / lactonisation (NCAL) reaction (Scheme 1). ${ }^{2}$ Further work from the Romo group has demonstrated the utility of ammonium and isothiouronium enolates generated from carboxylic acids in the stereoselective synthesis of $\beta$-lactones, ${ }^{3}$ including the application to the total synthesis of $(+)$-omphadiol $^{3 \mathrm{j}} \mathbf{1}$ and (-)-curcumalactone $\mathbf{2}$ (Scheme 1), amongst others. ${ }^{31}$<smiles>[R]C(CCC(=O)O)CC(=O)O</smiles>
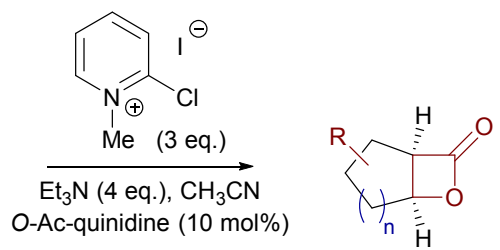

4 examples, $37-54 \%$ yield up to $92 \%$ ee

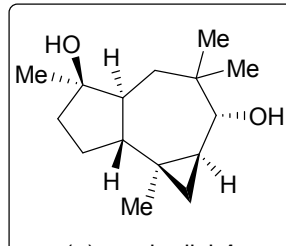

(+)-omphadiol 1

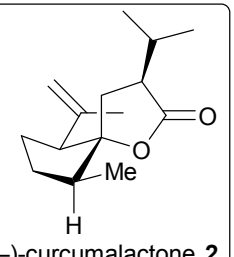

(-)-curcumalactone 2

Scheme 1 Romo and co-wokers' intramolecular NCAL reaction ${ }^{2}$ and select examples of natural products accessed using this reaction. ${ }^{3 \mathrm{j}, 31}$
We have previously explored the scope of the intramolecular reactions of ammonium and isothiouronium enolates, ${ }^{4}$ allowing access to dihydrobenzofurans, ${ }^{5} 2 H$-indenes, ${ }^{5 \mathrm{a}} \mathrm{THFs},{ }^{5 \mathrm{~b}}$ as well as pyrrolidines. $^{6}$ Intermolecular reactions have also been developed, with formal [2+2]- and [4+2]-cycloadditions between isothiouronium enolates and suitable electrophiles greatly expanding the range of valuable heterocyclic products that can be accessed directly from carboxylic acids. This has included dihydropyranones, ${ }^{5 \mathrm{a}, 7} \quad \beta$-lactams, ${ }^{8}$ dihydropyridinones, ${ }^{9}$ pyridines, ${ }^{10}$ 2-pyrones ${ }^{11}$ and a variety of acyclic products from further derivatisation of the heterocyclic cores. $^{12}$

Our previous synthesis of dihydropyridinones reacted isothiouronium enolates generated from carboxylic acids with $\mathrm{N}$-tosyl chalcone-derived ketimines in a Michael addition / lactamisation cascade (Scheme 2a). ${ }^{9}$ This afforded a range of dihydropyridinones in high yields and excellent stereoselectivity. In this methodology the $N$-tosyl substituent was employed exclusively in the ketimine Michael acceptor. Furthermore, the use of chalcones as the backbone of the ketimine, alongside the requirement for aryl or heteroaryl acetic acids, resulted in dihydropyridinones furnished with three (hetero)aryl substituents thereby constraining the scope of the hetercyclic products. To extend these studies, alternative ketimine backbones incorporating more versatile functional handles, whilst maintaining the reactivity of the Michael acceptor were explored. Herein we report the successful incorporation of ketimines derived from $\alpha, \beta$-unsaturated $\gamma$ ketoesters within this 
a) Previous Work (ref 9):

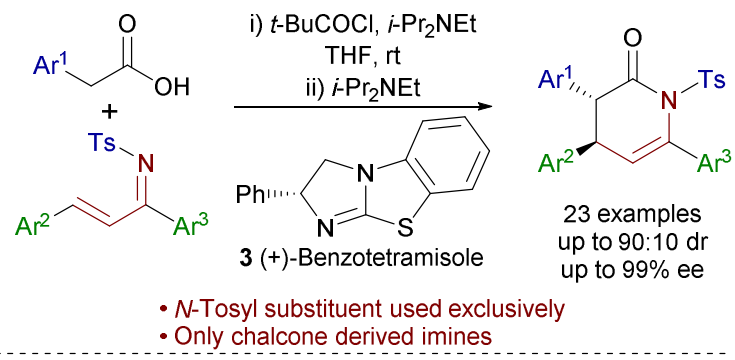

b) This Work

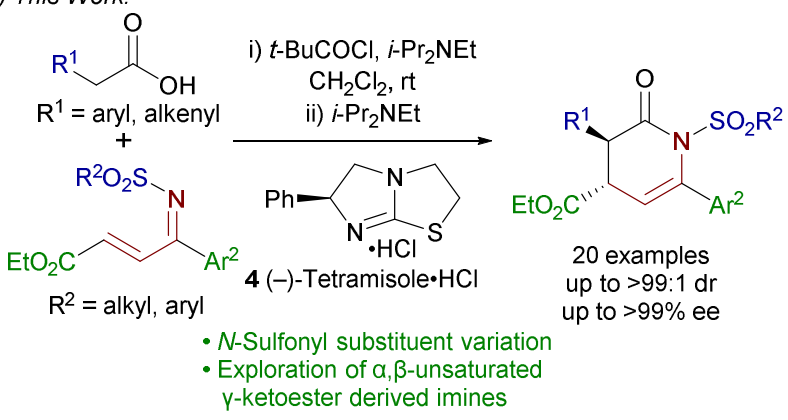

Scheme 2 Organocatalytic synthesis of dihydropyridinones

methodology, ${ }^{10,13}$ affording dihydropyridinone products containing ester substituents as functional handles (Scheme 2b). Extensive exploration of the sulfonyl substituent and the potential for derivatisation of the dihydropyridinone core is also described.

\section{Results and Discussion}

\section{Optimisation}

Initial studies showed that $\alpha, \beta$-unsaturated $\gamma$-ketimine ester 6 was a competent electrophile for intermolecular Michael addition / lactamisation with the isothiouronium enolate generated from phenylacetic acid $\mathbf{5}$ after in situ formation of a mixed anhydride using pivaloyl chloride and base, and reaction with achiral isothiourea DHPB 7. This allows formation of dihydropyridinone 8 in $65 \%$ isolated yield as an 88:12 mixture of diastereoisomers (Scheme 3a). In contrast, the related but isomeric Michael acceptor 9 resulted in a $c a$. 1:1 mixture of diastereoisomers in a poorer yield (Scheme $3 b){ }^{14}$

With reactivity and diastereoselectivity confirmed with Michael acceptor 6, a screen of common chiral isothiourea Lewis bases 3, 4 and 11 showed (-)-tetramisole $\cdot \mathrm{HCl} 4$ to be optimum in terms of both isolated yield and stereoselectivity (Table 1, entries 1-3). Examining the catalyst loading revealed that although increasing to $40 \mathrm{~mol} \% 4$ gave a higher isolated yield of $\mathbf{8}$, the diastereoselectivity suffered (entry 4). Conversely, lowering the loading to $10 \mathrm{~mol} \%$ resulted in incomplete conversion of 6 even after extended reaction times (entry 5). Next, the activating agent for generation of the in situ formed mixed anhydride was examined, showing that pivaloyl chloride was the best choice
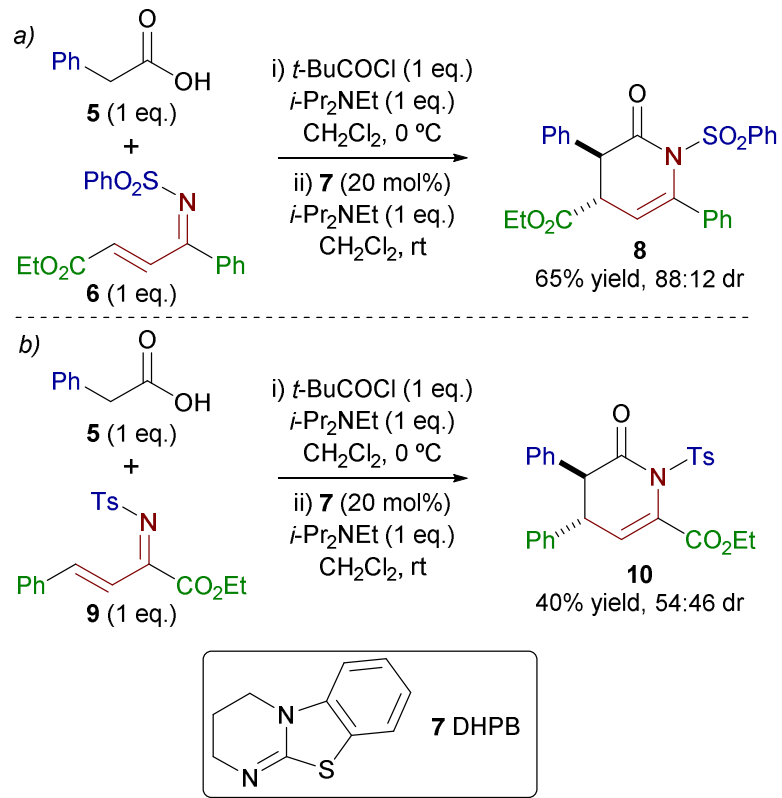

Scheme 3 Initial investigations

over a range of benzoyl chloride derivatives (entries 6-8). Tetramisole free base (entry 9) and excess $i$ - $\operatorname{Pr}_{2} \mathrm{NEt}$ (1.2 eq., entry 10) were examined, resulting in a slight reduction of diastereoselectivity and yield, respectively. Increasing the amount of carboxylic acid and pivaloyl chloride to two equivalents led to consistently higher isolated yields in a short reaction time, even under the more operationally simple nonanhydrous conditions of bench solvent and un-dried glassware (entries 11 and 12). Reducing the catalyst loading under these conditions was again found to be detrimental to the isolated yield of 8 (entry 13). The scalability of the reaction was examined with $5.0 \mathrm{mmol}$ of $\mathbf{6}$ affording $1.48 \mathrm{~g}(3.2 \mathrm{mmol})$ of 8 (entry 14) in near identical yield and stereoselectivity to that on 0.2 mmol scale ( $c f$. entry 12), demonstrated the ability of this methodology to deliver gram quantities of enantioenriched dihydropyridinones. 


\section{Table 1 Reaction Optimisation ${ }^{\mathrm{a}}$}
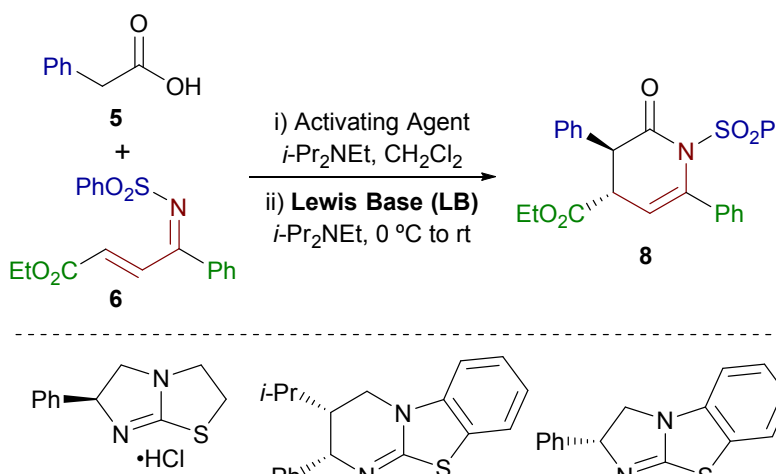<smiles>CC(C)[C@H]1CN2C(=N[C@@H]1c1ccccc1)Sc1c(F)cccc12</smiles>

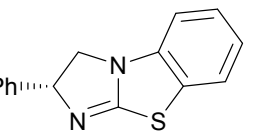

4 (-)-Tetramisole $\cdot H C$ 11 HyperBTM

$3(+)$-Benzotetramisole

\begin{tabular}{|c|c|c|c|c|c|}
\hline \multicolumn{2}{|c|}{ Entry $\mathbf{L B}(\mathrm{mol} \%)$} & $\begin{array}{l}\text { Activating } \\
\text { Agent (eq.) }\end{array}$ & \multicolumn{2}{|c|}{ time $(\mathrm{h})$ yield $(\%)^{b}$} & \multirow{2}{*}{$\begin{array}{c}\mathrm{dr}(\text { anti:syn) } \\
\text { ee }(\text { anti, \%) } \\
97: 3 / 98\end{array}$} \\
\hline 1 & $4(20)$ & $t-\mathrm{BuCOCl}(1)$ & 3 & 46 & \\
\hline 2 & $11(20)$ & $t-\mathrm{BuCOCl}(1)$ & 16 & 33 & $75: 25 / 95$ (ent) \\
\hline 3 & $3(20)$ & $t$-BuCOCl (1) & 16 & 28 & $>95: 5 / 98($ ent $)$ \\
\hline 4 & $4(40)$ & $t-\mathrm{BuCOCl}(1)$ & 3 & 75 & 93:7 / 98 \\
\hline 5 & $4(10)$ & $t$-BuCOCl (1) & 24 & 38 & $97: 3 / 98$ \\
\hline 6 & $4(20)$ & $\mathrm{PhCOCl}(1)$ & 3 & 32 & $91: 9 / 90$ \\
\hline 7 & $4(20)$ & $\mathrm{PMPCOCl}^{e}(1)$ & 24 & 17 & $90: 10 / 98$ \\
\hline 8 & $4(20)$ & $\operatorname{PNPCOCl}^{f}(1)$ & 3 & 29 & $83: 17$ / 94 \\
\hline $9^{g}$ & $4(20)$ & $t$-BuCOCl (1) & 3 & 62 & $93: 7 / 98$ \\
\hline $10^{h}$ & $4(20)$ & $t$-BuCOCl (1) & 3 & 31 & $98: 2 / 98$ \\
\hline $11^{i}$ & $4(20)$ & $t$-BuCOCl (2) & 1 & 62 & $93: 7 / 98$ \\
\hline $12^{i, j}$ & $4(20)$ & $t$-BuCOCl (2) & 1 & 65 & 92:8 / 98 \\
\hline $13^{i}$ & $4(10)$ & $t-\mathrm{BuCOCl}(2)$ & 3 & 45 & $97: 3 / 95$ \\
\hline $14^{k}$ & $4(20)$ & $t-\mathrm{BuCOCl}(2)$ & 1 & 64 & $92: 8 / 98$ \\
\hline
\end{tabular}

${ }^{a}$ Reaction Conditions: 5 ( 1 eq.), Activating agent ( 1 eq.), $i$ - $\operatorname{Pr}_{2} \mathrm{NEt}$ ( 1 eq.), $\mathrm{CH}_{2} \mathrm{Cl}_{2}$; then 6 (1 eq.), LB, $i$-Pr $\mathrm{Pr}_{2} \mathrm{NEt}$ (1 eq.); ${ }^{b}$ Isolated yield; ${ }^{c}$ Measured by ${ }^{1} \mathrm{H}$ NMR; ${ }^{d}$ Measured by chiral HPLC $;{ }^{e} \mathrm{PMP}=4$-methoxyphenyl; ${ }^{f} \mathrm{PNP}=4$ nitrophenyl; ${ }^{g}$ Tetramisole free base was used; ${ }^{h} i$-Pr ${ }_{2} \mathrm{NEt}$ (1.2 eq.) was used. 5 ( 2 eq.), activating agent ( 2 eq.) and $i$ - $\operatorname{Pr}_{2} \mathrm{NEt}(2$ eq. $)$ were used. ${ }^{j}$ Benchgrade solvents used; ${ }^{k} 5.0$ mmol of $\mathbf{6}$.

In an attempt to simplify the experimental procedure the possibility of using preformed homoanhydrides was explored (Scheme 4), with 2-phenylacetic anhydride 12 used in place of the combination of 2-phenylacetic acid and pivaloyl chloride. The purification of $\mathbf{8}$ was greatly simplified under this protocol, however lower diastereo- and enantioselectivity was observed with (-)-tetramisole $\cdot \mathrm{HCl}$ 4. HyperBTM 11 performed better in terms of enantioselectivity at the lower loading of $5 \mathrm{~mol} \%$, although 60:40 dr was observed. This may be owing to in situ epimerisation of the dihydropyridinone product, a conjecture that is supported by the high ee observed for the syndiastereoisomer in this case. Therefore, the use of pre-activated homoanhydrides was not explored further.

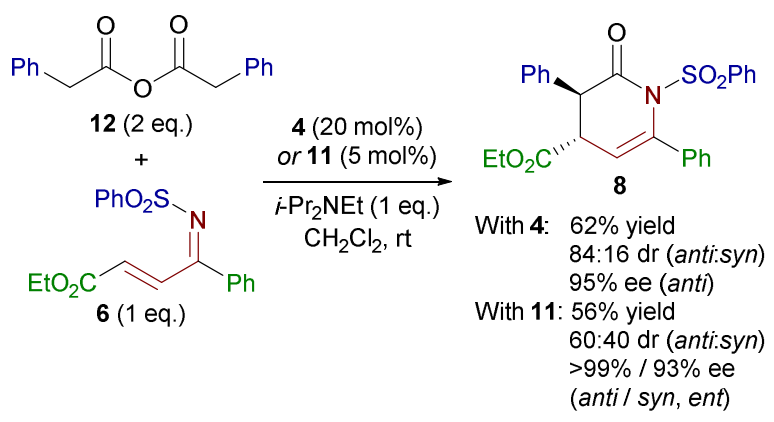

Scheme 4 Investigation of homoanhydride 12

\section{Exploration of the $\mathrm{N}$-Sulfonyl Substituent}

Having optimised the model system, exploration of the scope and limitations of this process required a general synthetic route to a range of ketimines. Their synthesis was achieved either directly from commercially available ethyl 3-benzoylacrylate 13 through $\mathrm{TiCl}_{4}$-mediated imine formation (Scheme $5 \mathrm{a}$ ), ${ }^{13,15}$ or efficiently in three steps from (+)-diethyl L-tartrate 15 via oxidative cleavage, Wittig reaction of the resulting aldehyde, ${ }^{16}$ and imine formation using $\mathrm{TiCl}_{4}$ (Scheme 5 b). In most cases the crude ketimines were purified through trituration and recrystallisation, thereby avoiding silica gel chromatography that leads to significant hydrolysis of these somewhat sensitive imines. ${ }^{17}$<smiles>CCOC(=O)/C=C/C(=O)c1ccccc1</smiles>

(L)-15

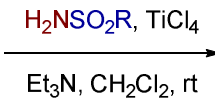

i) $\mathrm{H}_{5} \mathrm{IO}_{6}, \mathrm{Et}_{2} \mathrm{O}$, rt

ii)

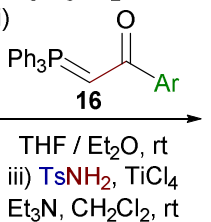

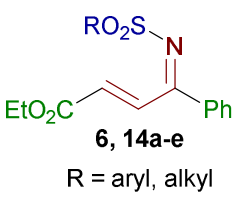

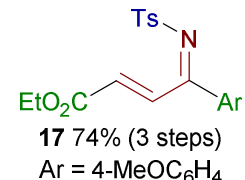

Scheme 5 Synthesis of $N$-sulfonyl ketimines

Initially, the scope of the sulfonyl group was examined to probe the effect of varying the steric and electronic properties of the $\mathrm{N}$-substituent on the Michael addition / lactamisation (Table 2). A range of different aryl groups was incorporated, including electron-donating tosyl 18 and $\mathrm{SO}_{2} \mathrm{PMP} \mathbf{1 9}$, and electronwithdrawing 4-nosyl 20 without significant variation in yield of dihydropyridinone. The sterically bulky 2,4,6triisopropylbenzene sulfonyl substituent could also be installed, although the isolated yield of $\mathbf{2 1}$ was much reduced. Pleasingly, an alkyl substituted sulfonyl ketimine also proved reactive, generating methanesulfonyl derivative 22 in good yield without loss of stereoselectivity. 
Table 2 Variation of the Sulfonyl Substituent

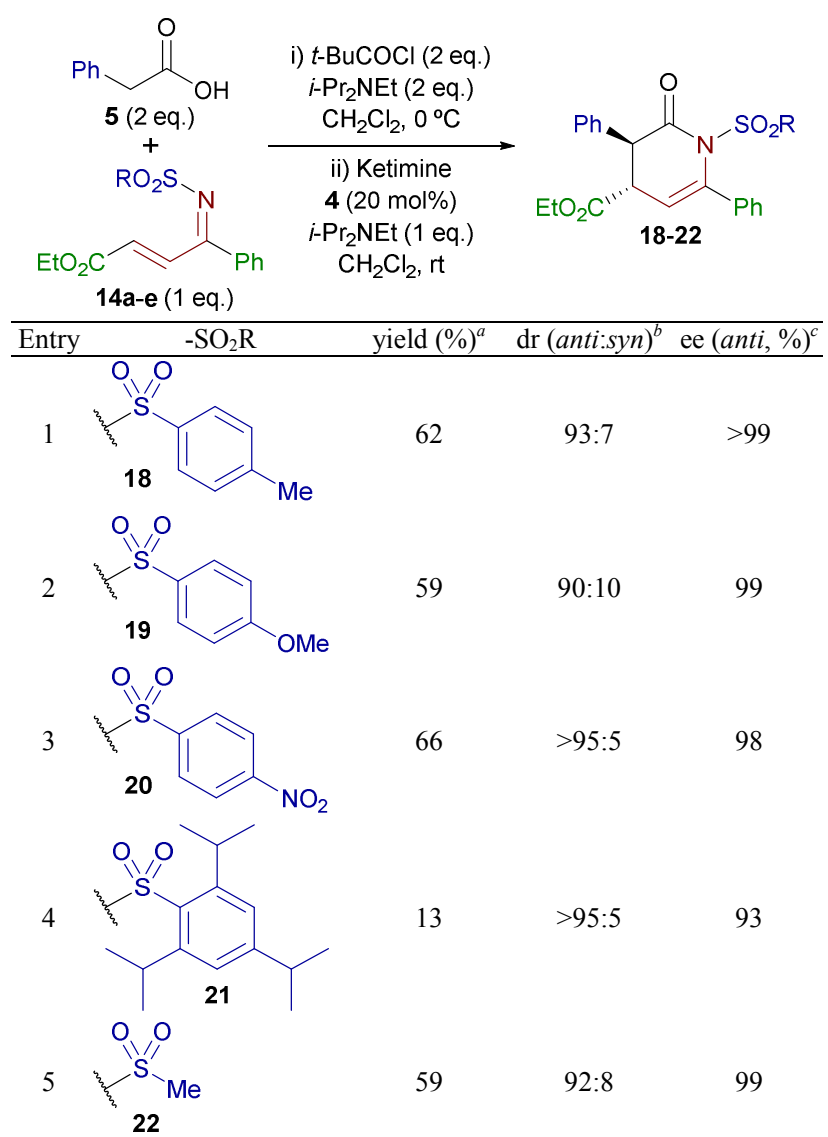

${ }^{a}$ Isolated yield; ${ }^{b}$ Measured by ${ }^{1} \mathrm{H}$ NMR; ${ }^{c}$ Measured by chiral HPLC.

\section{Exploration of the Acetic Acid Nucleophile Scope}

The scope of the acetic acid component was initially examined using $\alpha, \beta$-unsaturated benzenesulfonyl ketimine 6 (Scheme 6). Generally, the isolated yield of dihydropyridinone was higher when two equivalents of acetic acid was used rather than one (Method A c.f. Method B). Interestingly, product diastereoselectivity was marginally reduced in most cases when two equivalents of acetic acid was used. While the reason for this is not immediately clear, enantioselectivity remained excellent. Arylacetic acids containing para-, meta- and orthotolyl substituents were all incorporated in high yields and excellent stereoselectivities (23-25), demonstrating that orthosubstitution has no detrimental effect on the course of the reaction, unlike in our previous synthesis of dihydropyridinones $^{9}$ amongst other examples. ${ }^{7,12 a, 12 c}$ paraPhenyl as well as 1- and 2-naphthyl substitution gave the desired dihydropyridinones (26-28) in moderate yields, although stereoselectivities remained excellent. Electron-rich and halogenated aromatics could also be easily incorporated with excellent stereocontrol (29-33), with an improvement in isolated yield for the most electron-rich substituents ( $c f$. 8). The relative and absolute configuration of $\mathbf{3 1}$ was determined by Xray diffraction, with all other products assigned by analogy. ${ }^{18}$
Other substituents included the heteroaromatic 3-thiophenyl dihydropyridinone $\mathbf{3 4}$, which was formed without consequence as long as two equivalents of activated acetic acid were employed, and the 3-pentenoic acid derivative $35,{ }^{12 \mathrm{~d}}$ further expanding the scope of accessible motifs with functional handles for further derivatisation. Finally, the ketimine backbone was modified to incorporate a para-methoxyphenyl group in place of the phenyl substituent, resulting in the formation of $\mathbf{3 6}$ and $\mathbf{3 7}$ in moderate yield and high stereoselectivity. 


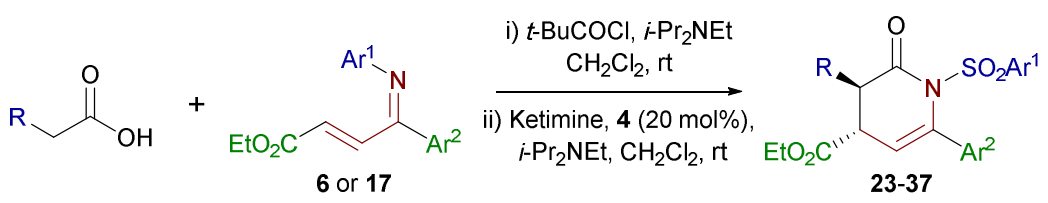<smiles>CCOC(=O)[C@@H]1C=C(c2ccccc2)N(OS(=O)(=O)c2ccccc2)C(=O)[C@H]1c1ccc(C)cc1</smiles>

A: $66 \%, 94: 6 \mathrm{dr}, 98 \%$ ee B: $50 \%,>99: 1 \mathrm{dr},>99 \%$ ee<smiles>CCO[C@H]1C=C(c2ccccc2)N(S(=O)(=O)c2ccccc2)C(=O)[C@H]1c1ccc2ccccc2c1</smiles>

27

A: $62 \%, 92: 8 \mathrm{dr}, 97 \%$ ee B: $37 \%,>99: 1 \mathrm{dr}, 95 \%$ ee<smiles>CCOC(=O)[C@H]1C=C(c2ccccc2)N(S(=O)(=O)c2ccccc2)C(=O)[C@H]1c1cccc(C)c1</smiles>

24

A: $72 \%, 93: 7 \mathrm{dr}, 99 \%$ ee<smiles>CCOC(=O)[C@H]1C=C(c2ccccc2)N(S(=O)(=O)c2ccccc2)C(=O)[C@H]1c1cccc2ccccc12</smiles>

A: $69 \%, 92: 8 \mathrm{dr}, 97 \%$ ee B: $32 \%, 96: 4 \mathrm{dr}, 96 \%$ ee

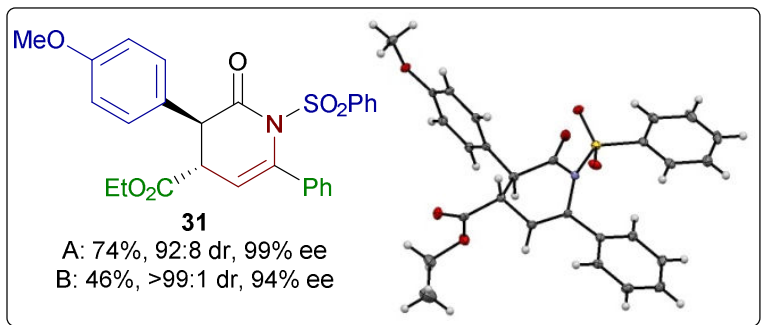<smiles>CCOC(=O)[C@H]1C=C(c2ccccc2)N(S(=O)(=O)c2ccccc2)C(=O)[C@H]1c1ccsc1</smiles>

34

A: $64 \%, 92: 8 \mathrm{dr}, 97 \%$ ee B: $10 \%, 84: 16 \mathrm{dr}, 85 \%$ ee<smiles>C/C=C/[C@H]1C(=O)N(S(=O)(=O)c2ccccc2)C(c2ccccc2)=C[C@H]1C(CC)OCC</smiles>

35

A: $53 \%, 88: 12 \mathrm{dr}, 98 \%$ ee<smiles>CCOC(=O)[C@H]1C=C(c2ccccc2)N(S(=O)(=O)c2ccccc2)C(=O)[C@H]1c1ccccc1</smiles>

A: $67 \%, 93: 7 \mathrm{dr}, 99 \%$ ee<smiles>CCOC(C)C1C=C(c2ccccc2)N(S(=O)(=O)c2ccccc2)C(=O)[C@H]1c1ccc(OC)c(OC)c1</smiles>

29

A: $78 \%, 97: 3 \mathrm{dr}, 99 \%$ ee B. $51 \%,>99: 1 \mathrm{dr},>99 \%$ ee<smiles>CCOC(=O)[C@H]1C=C(c2ccccc2)N(S(=O)(=O)c2ccccc2)C(=O)[C@H]1c1ccc(Cl)cc1</smiles>

A: $54 \%, 94: 6 \mathrm{dr}, 95 \%$ ee

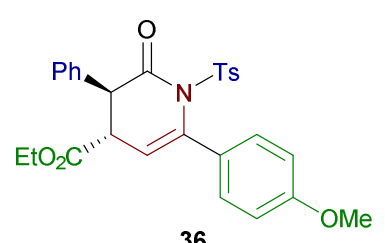

A: $58 \%, 90: 10 \mathrm{dr}, 99 \%$ ee<smiles>CCOC(=O)[C@H]1C=C(c2ccccc2)N(S(=O)(=O)c2ccccc2)C(=O)[C@H]1c1ccc(-c2ccccc2)cc1</smiles>

A: $76 \%, 92: 8 \mathrm{dr}, 97 \%$ ee B: $33 \%,>99: 1 \mathrm{dr}, 91 \%$ ee

$\mathrm{Me}_{2} \mathrm{~N}$<smiles>CCOC(=O)[C@H]1C=C(c2ccccc2)[C@@H](c2ccccc2)C(=O)N1S(=O)(=O)c1ccccc1</smiles>

A: $80 \%, 93: 7 \mathrm{dr}, 99 \%$ ee B: $70 \%, 93: 7 \mathrm{dr}, 98 \%$ ee

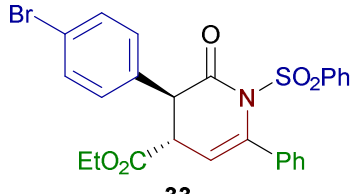

A: $60 \%, 94: 6 \mathrm{dr}, 95 \%$ ee B: $19 \%,>99: 1 \mathrm{dr}, 91 \%$ ee

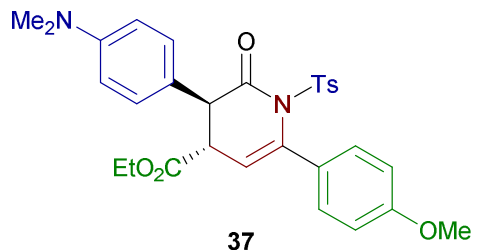

A: $47 \%, 88: 12 \mathrm{dr}, 91 \%$ ee

Scheme 6 Variation of acetic acid and ketimine and molecular representation of X-ray structure 31. Reaction conditons, A: Acetic acid (2 eq.), $t$-BuCOCl (2 eq.), $i$ $\operatorname{Pr}_{2} \mathrm{NEt}$ (2 eq.), $\mathrm{CH}_{2} \mathrm{Cl}_{2}$; then ketimine (1 eq.), 4 (20 mol\%), $i-\mathrm{Pr}_{2} \mathrm{NEt}$ (1 eq.), $\mathrm{CH}_{2} \mathrm{Cl}_{2}$. B: Acetic acid (1 eq.), $t$ - $\mathrm{BuCOCl}\left(1\right.$ eq.), $i$ - $\mathrm{Pr}_{2} \mathrm{NEt}\left(1\right.$ eq.), $\mathrm{CH}{ }_{2} \mathrm{Cl}{ }_{2}$; then ketimine (1 eq.), 4 (20 mol\%), $i-\mathrm{Pr}_{2} \mathrm{NEt}$ (1 eq.), $\mathrm{CH}_{2} \mathrm{Cl}_{2}$. Isolated yields; $\mathrm{dr}$ measured by ${ }^{1} \mathrm{H} \mathrm{NMR}$; ee measured by chiral HPLC.

\section{Mechanistic Rationale}

The proposed mechanism of the reaction is outlined in Scheme 7. Firstly, $N$-acylation of (-)-tetramisole 4 with mixed anhydride 38, formed in situ from phenylacetic acid and pivaloyl chloride, generates acyl isothiouronium $\mathbf{3 9}$. Deprotonation forms the $(Z)$-isothiouronium enolate, which undergoes a Michael addition / lactamisation cascade to give dihydropyridinone $\mathbf{4 2}$ with concomitant regeneration of the catalyst. A plausible pre-transition state assembly (43) that explains the relative and absolute configuration is shown. A stabilising interaction between the enolate oxygen and the sulfur of the isothiouronium ion locks the conformation of this species, ${ }^{17}$ thereby preventing $R e$-face attack through the stereodirecting phenyl group on tetramisole. 


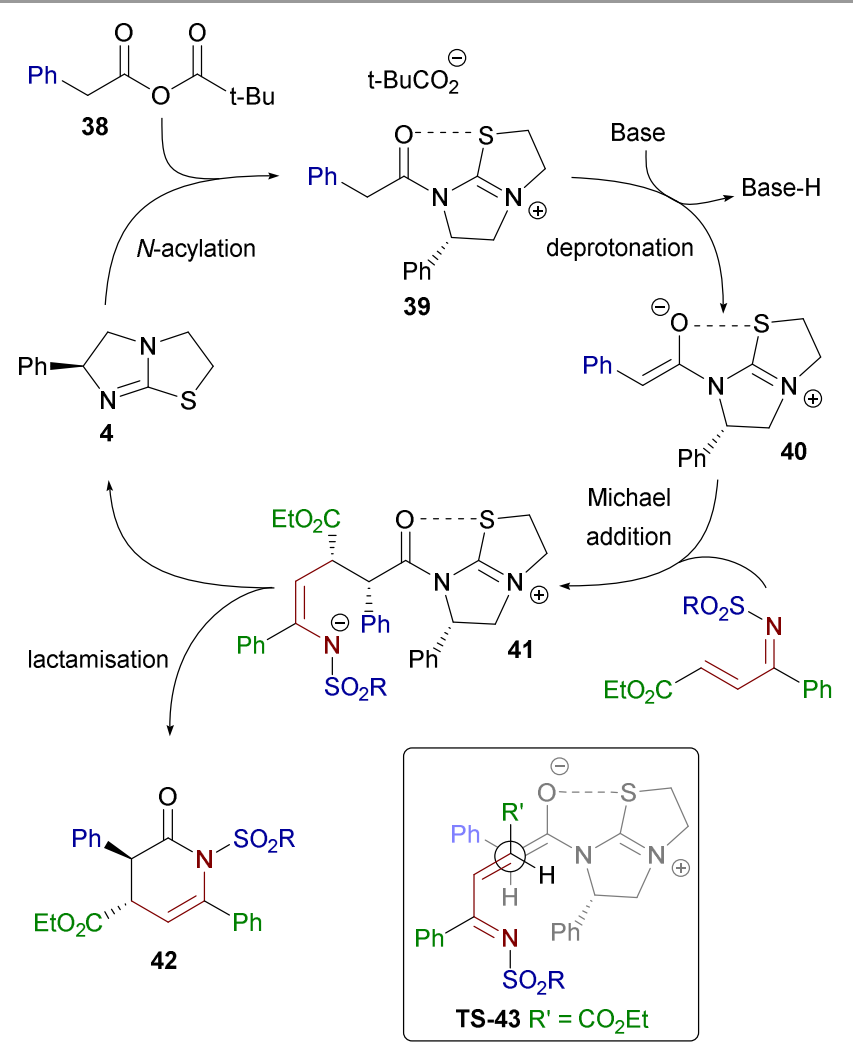

Scheme 7 Mechanistic rationale

\section{Derivatisation of the Dihydropyridinone Core}

The potential for derivatisation of the dihydropyridinones was next examined, first considering the possibility of converting the dihydropyridinone into a pyridine derivative. Dehydrogenation and desulfonylation of 8 using $\mathrm{Pd} / \mathrm{C}$ in the presence of sodium formate gave pyridone 44 (Scheme 8). Subsequent treatment of $\mathbf{4 4}$ with tosyl chloride gave tetrasubstituted pyridine $\mathbf{4 5}$ in excellent yield. This route is complementary to our previous work regarding isothiourea mediated pyridine formation, which generates 2,4,6trisubstituted pyridines ( $c f .46$ and 47) ${ }^{10-11}$ in contrast to the 2,3,4,6-tetrasubstituted pyridine formed here. Additionally, this methodology retains the 2-tosylate as a potential functional handle for further derivatisation. ${ }^{10}$

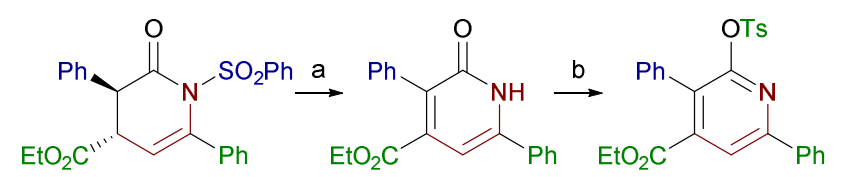

$4486 \%$
46 ref 10.

Scheme 8 Formation of tetrasubstituted pyridine 45. Reaction conditions: a) $\mathrm{Pd} / \mathrm{C}, \mathrm{HCO}_{2} \mathrm{Na}, \mathrm{DMF}, 60^{\circ} \mathrm{C}$; b) $\mathrm{NaH}, \mathrm{TsCl}$, DMF.
In contrast to $\mathrm{Pd} / \mathrm{C}$, selective desulfonylation without dehydrogenation was achieved using sodium naphthalenide, which gave unprotected dihydropyridinone $\mathbf{4 8}$ in modest yield without loss of stereoselectivity (Scheme 9a). Alternatively, bromination at the 5-position proceeded smoothly upon treatment with $\mathrm{Br}_{2}$ to give the fully substituted dihydropyridinone 49 in excellent yield (Scheme 9b). Ringopening of $\mathbf{8}$ proved challenging, for example catalytic DMAP in methanol returned only starting material. However, ringopening of $\mathbf{8}$ was possible using magnesium in methanol, which gave a moderate yield of keto-diester $\mathbf{5 0}$ in high enantioselectivity, alongside $60 \%$ of pyridone 44 from competing dehydrogenation / desufonylation under these more forcing conditions (Scheme 9c). Reduction of $\mathbf{8}$ with $\mathrm{LiAlH}_{4}$ followed by an acidic workup afforded a good yield of acyclic enamine-diol $\mathbf{5 1}$ as a single diastereoisomer without loss of enantiopurity (Scheme 9d). It is perhaps surprising that the $\mathrm{N}$ sulfonyl enamine is isolable under these conditions, but the good isolated yield indicates significant stability of this functionality. ${ }^{20}$

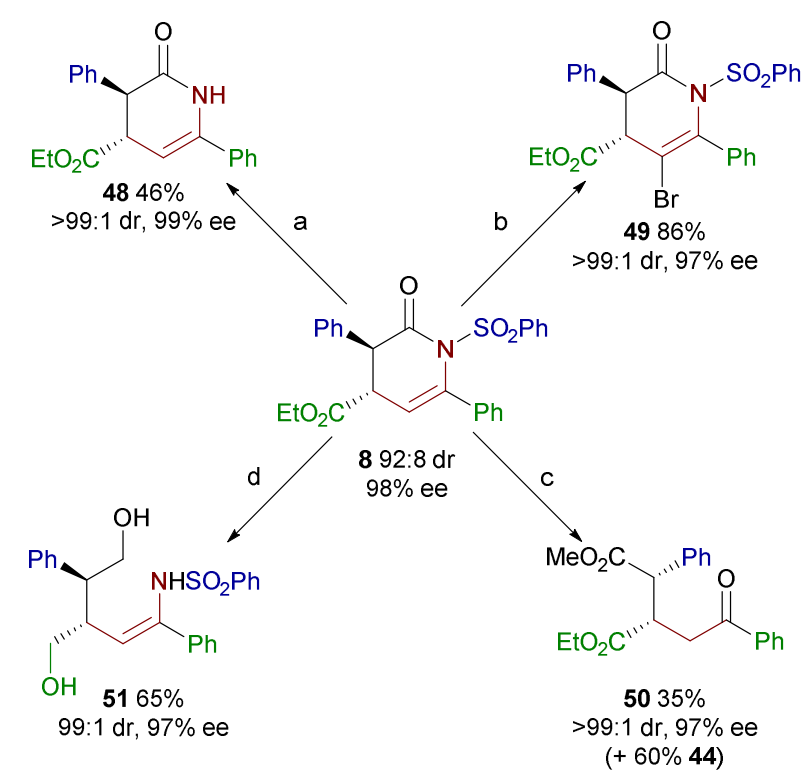

Scheme 9 Derivatisations of the dihydropyridinone core. Reaction conditions: a) $\mathrm{Na} /$ naphthalene (4 eq.), $-78{ }^{\circ} \mathrm{C}$; b) $\mathrm{Br}_{2}, i-\mathrm{Pr}_{2} \mathrm{NEt}$; c) $\mathrm{Mg} / \mathrm{MeOH}$; d) i) $2 \mathrm{M} \mathrm{LiAlH}_{4}$, THF; ii) $0.1 \mathrm{M} \mathrm{HCl}$.

While all attempts at homo- or heterogeneous hydrogenation of the $C-5,6$ double bond in $\mathbf{8}$ to form the 2-piperidone returned only starting material, epoxidation was successful with $\mathrm{m}$ CPBA. $^{21}$ This resulted in the formation of a single diastereoisomer of epoxide 52, containing four contiguous stereocentres (Scheme 10). The relative and absolute stereochemistry of $\mathbf{5 2}$ was confirmed through X-ray crystallography. ${ }^{22}$ 

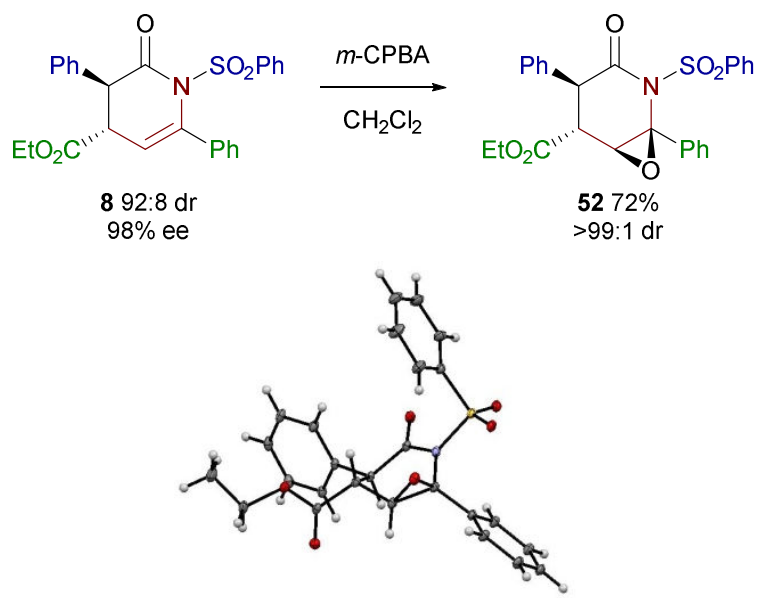

Scheme 10 Epoxidation of $\mathbf{8}$ and molecular representation of X-ray structure $\mathbf{5 2}$

\section{Conclusions}

In conclusion, the scope of the isothiourea catalysed Michael addition / lactamisation of aryl and alkenyl acetic acids with $\alpha, \beta$-unsaturated ketimines has been expanded, thereby allowing the synthesis of a variety of dihydropyridinone products. Derivatisation of the dihydropyridinone core has also been further demonstrated, affording a range of valuable skeletons from a single starting material. Further work in our laboratory is directed towards new Lewis base-catalysed reactions and exploring derivatisations of the functional building blocks they provide.

\section{Acknowledgements}

We thank the Royal Society for a University Research Fellowship (ADS), the EU (IEF for CS) and the European Research Council under the European Union's Seventh Framework Programme (FP7/2007-2013) ERC Grant Agreement No. 279850 (JET and CF) and the EPSRC grant numbers EP/J018139/1 (DSBD) and EP/K00445X/1 (EG) for funding. We also thank the EPSRC UK National Mass Spectrometry Facility at Swansea University.

\section{Experimental}

\section{General Information}

Anhydrous $\mathrm{CH}_{2} \mathrm{Cl}_{2}$ was obtained from an Mbraun SPS-800 system. Pet. ether is defined as petroleum ether $40-60{ }^{\circ} \mathrm{C}$. All other solvents and commercial reagents were used as supplied without further purification unless stated otherwise.

Room temperature (rt) refers to $20-25{ }^{\circ} \mathrm{C}$. Temperatures of $0{ }^{\circ} \mathrm{C}$ and $-78{ }^{\circ} \mathrm{C}$ were obtained using ice/water and $\mathrm{CO}_{2}$ (s)/acetone baths, respectively.

Analytical thin layer chromatography (TLC) was performed on pre-coated aluminium plates (Kieselgel $60 \mathrm{~F}_{254}$ silica). TLC visualisation was carried out with ultraviolet light $(254 \mathrm{~nm})$, followed by staining with a $1 \%$ aqueous $\mathrm{KMnO}_{4}$ solution and heating. Flash column chromatography was performed on Kieselgel 60 silica in the solvent system stated.

Melting points were recorded on an Electrothermal 9100 apparatus.

Optical rotations were measured on a Perkin Elmer Precisely/Model-341 polarimeter operating at the sodium D line with a $100 \mathrm{~mm}$ path cell at $20^{\circ} \mathrm{C}$.

HPLC analyses were obtained on a Shimadzu HPLC consisting of a DGU-20A5 degasser, LC-20AT liquid chromatograph, SIL-20AHT autosampler, CMB-20A communications bus module, SPD-M20A diode array detector and a CTO-20A column oven which allowed the temperature to be set from $25-40{ }^{\circ} \mathrm{C}$. Separation was achieved using a Chiralcel OD-H column or Chiralpak AD-H, and IA, columns. Authentic racemic samples of chiral products were synthesised using achiral DHPB 7 or racemic tetramisole $\cdot \mathrm{HCl} 4$.

Infrared spectra $\left(v_{\max }\right)$ were recorded on a Shimadzu IRAffinity-1 Fourier transform IR spectrophotometer as thin films using Pike MIRacle ATR accessory. Analysis was carried out using Shimadzu IRsolution v1.50 and only characteristic peaks are reported.

${ }^{1} \mathrm{H}$ and ${ }^{13} \mathrm{C}\left\{{ }^{1} \mathrm{H}\right\}$ spectra were acquired on either a Bruker Avance $300\left\{\delta_{\mathrm{H}}(300 \mathrm{MHz}), \delta_{\mathrm{C}}(75 \mathrm{MHz}), \delta_{\mathrm{F}}(282 \mathrm{MHz})\right\}$, a Bruker Avance II $400\left\{\delta_{\mathrm{H}}(400 \mathrm{MHz}), \delta_{\mathrm{C}}(101 \mathrm{MHz}), \delta_{\mathrm{F}}(376\right.$ $\mathrm{MHz})\}$ or a Bruker Ultrashield $500\left\{\delta_{\mathrm{H}}(500 \mathrm{MHz}), \delta_{\mathrm{C}}(126\right.$ $\left.\mathrm{MHz}), \delta_{\mathrm{F}}(471 \mathrm{MHz})\right\}$ spectrometer at ambient temperature in the deuterated solvent stated. Chemical shifts, $\delta$, are quoted in parts per million (ppm) and are referenced to the residual solvent peak. Coupling constants, $J$, are quoted in Hertz $(\mathrm{Hz})$ to the nearest $0.1 \mathrm{~Hz}$. The following abbreviations are used: s, singlet; d, doublet; t, triplet; q, quartet; m, multiplet; and br, broad.

Mass spectrometry $(\mathrm{m} / \mathrm{z})$ data were acquired by electrospray ionisation (ESI), electron impact (EI), chemical ionisation (CI), atmospheric solids analysis probe (ASAP), atmospheric pressure chemical ionisation (APCI) or nanospray ionisation (NSI) at the EPSRC National Mass Spectrometry Service Centre, Swansea.

General Procedure A: Synthesis of $\boldsymbol{\alpha}, \boldsymbol{\beta}$-unsaturated $\boldsymbol{\gamma}$ - $\boldsymbol{N}$-sulfonyl ketimines from ethyl 3-benzoylacrylate

A flame-dried flask containing a stirrer bar was charged with the requisite sulfonamide (1 eq.), ethyl 3-benzoylacrylate (1 eq.) and $\mathrm{CH}_{2} \mathrm{Cl}_{2}(\mathrm{ca} .0 .2 \mathrm{M})$. The resulting solution was stirred and cooled to $0{ }^{\circ} \mathrm{C}$ before $\mathrm{Et}_{3} \mathrm{~N}$ ( 2 eq.) was added followed by $\mathrm{TiCl}_{4}$ ( 1 eq.) dropwise. The reaction mixture was allowed to warm to rt, a reflux condenser fitted to the flask, and the reaction heated at reflux overnight. The solvent was removed in vacuo, the titanium salts precipitated with $\mathrm{Et}_{2} \mathrm{O}$ and the suspension filtered. The filtrate was concentrated in vacuo, the residue cooled in an ice bath, and triturated with a small amount of $\mathrm{Et}_{2} \mathrm{O}(c a .5 \mathrm{~mL} / \mathrm{g}$ ) with stirring. The resultant solid was collected by filtration and washed with further portions of cold $\mathrm{Et}_{2} \mathrm{O}$. The solid was dried in vacuo to leave the pure ketimines.

\section{General Procedure B: Synthesis of Dihydropyridinones}


A flask containing a stirrer bar was charged with $\mathrm{CH}_{2} \mathrm{Cl}_{2}$ (to give $0.4 \mathrm{M}$ acid] , aryl acetic acid (2 eq.), $i$ - $\operatorname{Pr}_{2} \mathrm{NEt}$ ( 2 eq.) and cooled to $0{ }^{\circ} \mathrm{C}$. Pivaloyl chloride ( 2 eq.) was added and the reaction stirred for 30 minutes. (-)-Tetramisole $\cdot \mathrm{HCl} 4$ (20 mol\%) was added followed by the ketimine (1 eq.) and additional $i$ - $\mathrm{Pr}_{2} \mathrm{NEt}$ ( 1 eq.) in $\mathrm{CH}_{2} \mathrm{Cl}_{2}$ (to give $0.4 \mathrm{M}$ of ketimine). The reaction was allowed to warm to $\mathrm{rt}$ and stirred until complete by TLC. The reaction was quenched with $0.1 \mathrm{M}$ $\mathrm{HCl}(\sim 20 \mathrm{~mL} / \mathrm{mmol}$ acid$)$, the layers separated and the aqueous layer extracted with $\mathrm{CH}_{2} \mathrm{Cl}_{2}(2 \times$ eq. vol. $)$. The combined organics were dried over $\mathrm{MgSO}_{4}$, the solvent removed in vacuo on a rotary evaporator $\left(<30{ }^{\circ} \mathrm{C}\right.$ bath temp.), and the residue purified by flash chromatography in the solvent system stated.

\section{Preparation of Ketimines}

\section{(2E,4E)-Ethyl 4-phenyl-4-((phenylsulfonyl)imino)but-2-} enoate $6^{13 a}$

Following general procedure $\mathrm{A}$, the reaction of ethyl 3benzoylacrylate $13(1.84 \mathrm{~mL}, 10 \mathrm{mmol})$, benzenesulfonamide $(1.57$ $\mathrm{g}, 10.0 \mathrm{mmol}), \mathrm{NEt}_{3}(2.8 \mathrm{~mL}, 20 \mathrm{mmol})$ and $\mathrm{TiCl}_{4}(1.1 \mathrm{~mL}, 10$ mmol) in $\mathrm{CH}_{2} \mathrm{Cl}_{2}(60 \mathrm{~mL})$ gave the title compound after trituration as white crystals $(2.04 \mathrm{~g}, 6.0 \mathrm{mmol}, 60 \%)$; m $51-52{ }^{\circ} \mathrm{C}\left\{\right.$ Lit. $^{13 \mathrm{a}} 51-$ $\left.53{ }^{\circ} \mathrm{C}\right\} ;{ }^{1} \mathrm{H}$ NMR $\left(500 \mathrm{MHz}, \mathrm{CDCl}_{3}\right) \delta_{\mathrm{H}}: 1.35(3 \mathrm{H}, \mathrm{t}, J$ 7.0, $\left.\mathrm{OCH}_{2} \mathrm{CH}_{3}\right), 4.30\left(2 \mathrm{H}, \mathrm{q}, J 6.3, \mathrm{OCH}_{2} \mathrm{CH}_{3}\right), 6.24(1 \mathrm{H}, \mathrm{d}, J 16.2$, $\mathrm{C}(2) H), 7.44(2 \mathrm{H}, \mathrm{t}, J 7.7, \mathrm{Ph} H), 7.51-7.56(3 \mathrm{H}, \mathrm{m}, \mathrm{Ph} H), 7.62(1 \mathrm{H}$, t, $J$ 7.4, $\mathrm{Ph} H), 8.03(2 \mathrm{H}, \mathrm{s}, \mathrm{Ph} H), 8.24(1 \mathrm{H}, \mathrm{d}, J 16.1, \mathrm{C}(3) H)$. Data consistent with literature. ${ }^{13 a}$

\section{(2E,3E)-Ethyl 4-phenyl-2-(tosylimino)but-3-enoate $9^{23}$}

The starting ketone, (E)-ethyl 2-oxo-4-phenylbut-3-enoate, was prepared according the procedure outlined by Smith et al. ${ }^{\text {a }}$

Following general procedure A, the reaction of the above ketone (2.0 g, $9.8 \mathrm{mmol}), p$-toluenesulfonamide (1.68 g, $9.8 \mathrm{mmol}^{2}, \mathrm{NEt}_{3}(2.8$ $\mathrm{mL}, 19.6 \mathrm{mmol})$ and $\mathrm{TiCl}_{4}(1.1 \mathrm{~mL}, 9.8 \mathrm{mmol})$ in $\mathrm{CH}_{2} \mathrm{Cl}_{2}(60 \mathrm{~mL})$ gave 9 after purification by flash chromatography (EtOAc / pet. ether, 1:5) as a brown oil (1.51 g, $4.2 \mathrm{mmol}, 43 \%)$; ${ }^{1} \mathrm{H}$ NMR (300 $\left.\mathrm{MHz}, \mathrm{CDCl}_{3}\right) \delta_{\mathrm{H}}: 1.48\left(3 \mathrm{H}, \mathrm{t}, J\right.$ 7.1, $\left.\mathrm{OCH}_{2} \mathrm{CH}_{3}\right), 2.43(3 \mathrm{H}, \mathrm{s}$, $\left.\mathrm{NTsCH}_{3}\right), 4.55\left(2 \mathrm{H}, \mathrm{q}, J 7.1, \mathrm{OCH}_{2} \mathrm{CH}_{3}\right), 6.82(1 \mathrm{H}, \mathrm{d}, J 16.5$, $\mathrm{C}(2) H), 7.29-7.56(8 \mathrm{H}, \mathrm{m}, \mathrm{Ar} H$ and $\mathrm{C}(3) H), 7.90(2 \mathrm{H}, \mathrm{d}, J$ 8.3, $\mathrm{Ar} H)$. Data consistent with literature. ${ }^{23}$

\section{$(2 E, 4 E)$-Ethyl 4-phenyl-4-(tosylimino)but-2-enoate 14a}

Following general procedure $A$, the reaction of ethyl 3benzoylacrylate $(1.84 \mathrm{~mL}, 10 \mathrm{mmol}), p$-toluenesulfonamide $(1.68 \mathrm{~g}$, $10.0 \mathrm{mmol}), \mathrm{NEt}_{3}(2.8 \mathrm{~mL}, 20 \mathrm{mmol})$ and $\mathrm{TiCl}_{4}(1.1 \mathrm{~mL}, 10 \mathrm{mmol})$ in $\mathrm{CH}_{2} \mathrm{Cl}_{2}(60 \mathrm{~mL})$ gave the title compound after trituration $(1.22 \mathrm{~g}$, $3.4 \mathrm{mmol}, 34 \%$ ) as white crystals; $\mathrm{mp} 53-54{ }^{\circ} \mathrm{C} ; v_{\max }$ (ATR): 2938 , $1714,1545,1304,1157,1094 ;{ }^{1} \mathrm{H}$ NMR $\left(500 \mathrm{MHz}, \mathrm{CDCl}_{3}\right) \delta_{\mathrm{H}}: 1.35$ $\left(3 \mathrm{H}, \mathrm{t}, J 6.8, \mathrm{OCH}_{2} \mathrm{CH}_{3}\right), 2.44\left(3 \mathrm{H}, \mathrm{s}, \mathrm{NTsCH}_{3}\right), 4.30(2 \mathrm{H}, \mathrm{q}, J 6.6$, $\left.\mathrm{OCH}_{2} \mathrm{CH}_{3}\right), 6.22(1 \mathrm{H}, \mathrm{d}, J 16.2, \mathrm{C}(2) H), 7.34(2 \mathrm{H}, \mathrm{d}, J$ 7.9, $\mathrm{Ar} H)$, $7.43(2 \mathrm{H}, \mathrm{t}, J$ 7.6, $\mathrm{ArH}), 7.56(1 \mathrm{H}, \mathrm{t}, J 7.4, \operatorname{Ar} H), 7.72(2 \mathrm{H}, \mathrm{s}, \mathrm{Ar} H)$, 7.84-7.96 (2H, br s, ArH), $8.24(1 \mathrm{H}, \mathrm{d}, J 16.1, \mathrm{C}(3) H) ;{ }^{13} \mathrm{C}\left\{{ }^{1} \mathrm{H}\right\}$ NMR $\left(101 \mathrm{MHz}, \mathrm{CDCl}_{3}\right) \delta_{\mathrm{C}}: 14.1,21.5,61.5,127.4,128.5,129.5$, 130.1, 132.8, 133.3, 135.7, 136.7, 137.6, 144.0, 164.5, 174.5; HRMS (ESI+): $\mathrm{C}_{19} \mathrm{H}_{20} \mathrm{NO}_{4} \mathrm{~S}[\mathrm{M}+\mathrm{H}]^{+}$found 358.1107 , requires 358.1108 $(-0.2 \mathrm{ppm})$.

$(2 E, 4 E)$-Ethyl

phenylbut-2-enoate $14 \mathrm{~b}$

Following general procedure A, the reaction of ethyl 3- benzoylacrylate $\quad(0.92 \quad \mathrm{~mL}, \quad 5.0 \quad \mathrm{mmol})$, methoxybenzenesulfonamide $(0.94 \mathrm{~g}, 5.0 \mathrm{mmol}), \mathrm{NEt}_{3}(1.39 \mathrm{~mL}$, $10.0 \mathrm{mmol})$ and $\mathrm{TiCl}_{4}(0.55 \mathrm{~mL}, 5.0 \mathrm{mmol})$ in $\mathrm{CH}_{2} \mathrm{Cl}_{2}(30 \mathrm{~mL})$, gave the title compound after trituration $(0.95 \mathrm{~g}, 2.5 \mathrm{mmol}, 50 \%)$ as an orange solid; $\mathrm{mp} 76-78{ }^{\circ} \mathrm{C}$; $v_{\max }$ (ATR): 2985, 1716, 1552, 1494, $1311,1255,1184,1143,1089,1002,975 ;{ }^{1} \mathrm{H}$ NMR $(500 \mathrm{MHz}$, $\left.\mathrm{CDCl}_{3}\right) \delta_{\mathrm{H}}: 1.35\left(1 \mathrm{H}, \mathrm{t}, J 6.4, \mathrm{OCH}_{2} \mathrm{CH}_{3}\right), 3.38\left(3 \mathrm{H}, \mathrm{s}, \operatorname{ArOCH}{ }_{3}\right)$, 4.18-4.40 (2H, m, $\left.\mathrm{OCH}_{2} \mathrm{CH}_{3}\right), 6.21(1 \mathrm{H}, \mathrm{d}, J 16.2, \mathrm{C}(2) H), 7.00(2 \mathrm{H}$, d, $J 8.3, \mathrm{Ph} H), 7.43(2 \mathrm{H}, \mathrm{t}, J 7.5, \mathrm{Ph} H), 7.56(1 \mathrm{H}, \mathrm{t}, J 7.2, \mathrm{Ph} H)$, 7.63-7.80 (2H, m, $\left.\mathrm{NSO}_{2} \operatorname{ArC}(3) H\right), \quad 7.97 \quad(2 \mathrm{H}, \quad \mathrm{d}, \quad J$ 3.7, $\left.\mathrm{NSO}_{2} \mathrm{ArC}(2) H\right), 8.25(1 \mathrm{H}, \mathrm{d}, J 16.2, C(3) H) ;{ }^{13} \mathrm{C}\left\{{ }^{1} \mathrm{H}\right\}$ NMR $(126$ $\left.\mathrm{MHz}, \mathrm{CDCl}_{3}\right) \delta_{\mathrm{C}}: 14.3,55.8,61.7,114.2,128.7,129.7,130.3,132.3$, $132.9,133.4,136.0,136.9,160.8,163.4,170.3$; HRMS (ESI+): $\mathrm{C}_{19} \mathrm{H}_{20} \mathrm{NO}_{5} \mathrm{~S}[\mathrm{M}+\mathrm{H}]^{+}$found 374.1054 , requires $374.1057 \quad(-0.7$ ppm).

(2E,4E)-Ethyl 4-(((4-nitrophenyl)sulfonyl)imino)-4-phenylbut-2enoate 14c

Following general procedure $\mathrm{A}$, the reaction of ethyl 3benzoylacrylate $(0.92 \mathrm{~mL}, 5.0 \mathrm{mmol})$, 4-nitrobenzenesulfonamide $(1.01 \mathrm{~g}, 5.0 \mathrm{mmol}), \mathrm{NEt}_{3}(1.39 \mathrm{~mL}, 10.0 \mathrm{mmol})$ and $\mathrm{TiCl}_{4}(0.55 \mathrm{~mL}$, $5.0 \mathrm{mmol})$ in $\mathrm{CH}_{2} \mathrm{Cl}_{2}(30 \mathrm{~mL})$, gave the title compound after trituration as a yellow solid (1.32 g, $3.4 \mathrm{mmol}, 68 \%)$; mp $84-86^{\circ} \mathrm{C}$; $v_{\max }$ (ATR): 3115, 2978, 1724, 1519, 1294, 1271, 1184, 1161, 1147, $1091,1029,983 ;{ }^{1} \mathrm{H}$ NMR $\left(400 \mathrm{MHz}, \mathrm{CDCl}_{3}\right) \delta_{\mathrm{H}}: 1.35(1 \mathrm{H}, \mathrm{t}, J 7.1$, $\left.\mathrm{OCH}_{2} \mathrm{CH}_{3}\right), 4.18\left(2 \mathrm{H}, \mathrm{q}, J 7.1, \mathrm{OCH}_{2} \mathrm{CH}_{3}\right), 6.32(1 \mathrm{H}, \mathrm{d}, J 16.1$, $\mathrm{C}(2) H), 7.47(2 \mathrm{H}, \mathrm{d}, J 7.8, \mathrm{Ph} H), 7.61(1 \mathrm{H}, \mathrm{t}, J 7.5, \mathrm{Ph} H), 7.65-7.80$ $(2 \mathrm{H}, \mathrm{m}, \mathrm{Ph} H), 8.20-8.27\left(3 \mathrm{H}, \mathrm{m}, \mathrm{NSO}_{2} \mathrm{ArC}(2) H\right.$ and $\left.\mathrm{C}(3) H\right), 8.39$ $\left(2 \mathrm{H}, \mathrm{d}, J 8.8, \mathrm{NSO}_{2} \mathrm{ArC}(3) H\right) ;{ }^{13} \mathrm{C}\left\{{ }^{1} \mathrm{H}\right\} \mathrm{NMR}\left(101 \mathrm{MHz}, \mathrm{CDCl}_{3}\right) \delta_{\mathrm{C}}$ : 14.3, 61.9, 124.4, 128.8, 129.0, 130.4, 134.0, 134.1, 135.3, 146.4, 150.4, 164.5, 176.6; HRMS (ESI+): $\mathrm{C}_{18} \mathrm{H}_{17} \mathrm{~N}_{2} \mathrm{O}_{6} \mathrm{~S}[\mathrm{M}+\mathrm{H}]^{+}$found 389.0802 , requires $389.0802( \pm 0.0 \mathrm{ppm})$.

\section{$(2 E, 4 E)$-Ethyl}

4-phenyl-4-(( $(2,4,6-$

\section{triisopropylphenyl)sulfonyl)imino)but-2-enoate 14d}

Following general procedure $\mathrm{A}$, the reaction of ethyl 3benzoylacrylate $\quad(7.3 \quad \mathrm{~mL}, \quad 40.0 \quad \mathrm{mmol}), \quad 2,4,6-$ triisopropylbenzenesulfonamide (11.34 g, $40.0 \mathrm{mmol}), \mathrm{NEt}_{3}(11.2$ $\mathrm{mL}, 80.0 \mathrm{mmol})$ and $\mathrm{TiCl}_{4}(4.4 \mathrm{~mL}, 5.0 \mathrm{mmol})$ in $\mathrm{CH}_{2} \mathrm{Cl}_{2}(120 \mathrm{~mL})$, gave the title compound after trituration as a white solid $(14.50 \mathrm{~g}$, $30.8 \mathrm{mmol}, 77 \%$ ); mp $104-106{ }^{\circ} \mathrm{C}$; $v_{\max }$ (ATR): 2964, 1720, 1622, 1150,$939 ;{ }^{1} \mathrm{H}$ NMR $\left(500 \mathrm{MHz}, \mathrm{CDCl}_{3}\right) \delta_{\mathrm{H}}: 1.25(18 \mathrm{H}$, app t, $J 6.9$, $\left.\operatorname{Ar}\left(\mathrm{CH}\left(\mathrm{CH}_{3}\right)_{2}\right)_{3}\right), 1.31\left(3 \mathrm{H}, \mathrm{t}, J 7.1, \mathrm{OCH}_{2} \mathrm{CH}_{3}\right), 2.90(1 \mathrm{H}$, septet, $J$ 6.8, $\left.\quad \operatorname{ArC}(4) \mathrm{CH}\left(\mathrm{CH}_{3}\right)_{2}\right), \quad 4.20-4.29 \quad\left(4 \mathrm{H}, \quad \mathrm{m}, \quad \mathrm{OCH}_{2} \mathrm{CH}_{3}\right.$ and $\left.\mathrm{ArC}(2) \mathrm{C} H\left(\mathrm{CH}_{3}\right)_{2}\right), 6.09(1 \mathrm{H}, \mathrm{d}, J 16.3, \mathrm{C}(2) H), 7.16(2 \mathrm{H}, \mathrm{s}$, $\left.\mathrm{NSO}_{2} \mathrm{ArC}(3) H\right), 7.42(2 \mathrm{H}, \mathrm{t}, J$ 7.1, $\mathrm{Ph} H), 7.49-7.61(1 \mathrm{H}, \mathrm{m}, \mathrm{Ph} H)$, $7.76(2 \mathrm{H}, \mathrm{d}, J 6.1, \mathrm{Ph} H), 8.05(1 \mathrm{H}, \mathrm{d}, J 16.3, \mathrm{C}(3) H) ;{ }^{13} \mathrm{C}\left\{{ }^{1} \mathrm{H}\right\} \mathrm{NMR}$ $\left(126 \mathrm{MHz}, \mathrm{CDCl}_{3}\right) \delta_{\mathrm{C}}: 14.3,23.8,24.8,30.2,34.4,61.6,123.7$, $128.8,130.3,132.4,133.3,134.2,136.2,136.7,150.0,153.3,164.6$, 173.8; HRMS (ESI+): $\mathrm{C}_{35} \mathrm{H}_{41} \mathrm{~N}_{1} \mathrm{O}_{5} \mathrm{Na}_{1} \mathrm{~S}_{1} \quad\left([\mathrm{M}+\mathrm{Na}]^{+}\right)$, found 610.2603 requires $610.2592(-1.2 \mathrm{ppm})$.

(2E,4E)-Ethyl 4-((methylsulfonyl)imino)-4-phenylbut-2-enoate $14 \mathrm{e}$

Following general procedure $\mathrm{A}$, the reaction of ethyl 3benzoylacrylate $(0.92 \mathrm{~mL}, 5.0 \mathrm{mmol})$, methanesulfonamide $(0.48 \mathrm{~g}$, $5.0 \mathrm{mmol}), \mathrm{NEt}_{3}(1.39 \mathrm{~mL}, 10.0 \mathrm{mmol})$ and $\mathrm{TiCl}_{4}(0.55 \mathrm{~mL}, 5.0$ $\mathrm{mmol})$ in $\mathrm{CH}_{2} \mathrm{Cl}_{2}(30 \mathrm{~mL})$, gave the title compound after trituration as an orange solid $(0.87 \mathrm{~g}, 3.1 \mathrm{mmol}, 62 \%) ; \operatorname{mp~} 76-78{ }^{\circ} \mathrm{C} ; v_{\max }$ (ATR): 2991, 1718, 1639, 1579, 1554, 1442, 1365, 1309, 1292, $1269,1184,1134,1026,970 ;{ }^{1} \mathrm{H}$ NMR $\left(500 \mathrm{MHz}, \mathrm{CDCl}_{3}\right) \delta_{\mathrm{H}}: 1.32$ $\left(3 \mathrm{H}, \mathrm{t}, J 7.1, \mathrm{OCH}_{2} \mathrm{CH}_{3}\right), 3.26\left(3 \mathrm{H}, \mathrm{s}, \mathrm{SO}_{2} \mathrm{CH}_{3}\right), 4.28(2 \mathrm{H}, \mathrm{q}, J 7.1$, 
$\left.\mathrm{OCH}_{2} \mathrm{CH}_{3}\right), 6.25(1 \mathrm{H}, \mathrm{d}, J 16.2, \mathrm{C}(2) H), 7.47(2 \mathrm{H}, \mathrm{t}, J$ 7.8, $\mathrm{Ph} H)$, $7.60(1 \mathrm{H}, \mathrm{t}, J 7.1, \mathrm{Ph} H), 7.76(2 \mathrm{H}, \mathrm{d}, J 7.2, \mathrm{Ph} H), 8.13(1 \mathrm{H}, \mathrm{d}, J$ 16.2, C $(3) H) ;{ }^{13} \mathrm{C}\left\{{ }^{1} \mathrm{H}\right\} \mathrm{NMR}\left(126 \mathrm{MHz}, \mathrm{CDCl}_{3}\right) \delta_{\mathrm{C}}: 14.1,43.0,61.6$, $128.8,130.1,133.3,133.5,135.7,136.2,164.5,175.3$; HRMS (ESI+): $\mathrm{C}_{13} \mathrm{H}_{16} \mathrm{NO}_{4} \mathrm{~S}[\mathrm{M}+\mathrm{H}]^{+}$, found 282.0792, requires 282.0795 $(-0.9 \mathrm{ppm})$.

\section{(2E,4E)-Ethyl 4-(4-methoxyphenyl)-4-(tosylimino)but-2-enoate} $17^{13 a}$

A flame-dried flask containing a stirrer bar was charged with $(+)-$ diethyl (L)-tartrate $15(0.66 \mathrm{~mL}, 3.0 \mathrm{mmol}, 1$ eq. $)$ and anhydrous $\mathrm{Et}_{2} \mathrm{O}(6 \mathrm{~mL})$. Periodic acid $(0.68 \mathrm{~g}, 3.0 \mathrm{mmol}, 1$ eq. $)$ was added and the reaction stirred for $3 \mathrm{~h}$ at $\mathrm{rt}$. The suspension was filtered through cotton wool into a second flask, washing with anhydrous THF (7.5 $\mathrm{mL})$. The filtrate was treated with $\mathrm{MgSO}_{4}(1.0 \mathrm{~g})$ and the resulting suspension cooled to $0 \quad{ }^{\circ} \mathrm{C}$. 1-(4-Methoxyphenyl)-2(triphenylphosphoranylidene)ethanone 16 (1.85 g, $4.5 \mathrm{mmol}, 1.5$ eq.) was then added in one portion. The reaction was allowed to warm slowly to rt and stirred overnight. The solution was filtered and the solvent was removed in vacuo before purification of the crude material by flash chromatography on silica gel $\left(\mathrm{Et}_{2} \mathrm{O} /\right.$ hexane $0: 100$ to $30: 70)$ to give $(E)$-ethyl 4-(4-methoxyphenyl)-4-oxobut-2-enoate $17(0.92 \mathrm{~g}, 3.9 \mathrm{mmol}, 87 \%)$ as a yellow solid.

Following general procedure A with a modified work up, the reaction of $(E)$-ethyl 4-(4-methoxyphenyl)-4-oxobut-2-enoate $(0.77$ g, $3.3 \mathrm{mmol})$, $p$-toluenesulfonamide $\left(0.57 \mathrm{~g}, 3.3 \mathrm{mmol}^{2}, \mathrm{NEt}_{3}(0.92\right.$ $\mathrm{mL}, 6.6 \mathrm{mmol})$ and $\mathrm{TiCl}_{4}(0.36 \mathrm{~mL}, 3.3 \mathrm{mmol})$ in $\mathrm{CH}_{2} \mathrm{Cl}_{2}(17 \mathrm{~mL})$ was followed by aqueous work up. The reaction mixture was quenched with water $(30 \mathrm{~mL})$ and extracted with EtOAc $(\times 3)$, dried over $\mathrm{MgSO}_{4}$, filtered, and concentrated in vacuo. The crude residue was purified by Biotage ${ }^{\circledR}$ Isolera $^{\mathrm{TM}} 4$ [SNAP Ultra $25 \mathrm{~g}, 75 \mathrm{mLmin}^{-}$ 1, hexane:EtOAc $(75: 252 \mathrm{CV}, 57: 43 \quad 7 \mathrm{CV})]$ to give the title compound $17(1.08 \mathrm{~g}, 2.8 \mathrm{mmol}, 85 \%)$ as a thick yellow oil; ${ }^{1} \mathrm{H}$ NMR $\left(500 \mathrm{MHz}, \mathrm{CDCl}_{3}\right) \delta_{\mathrm{H}}: 1.34\left(3 \mathrm{H}, \mathrm{t}, J 6.7, \mathrm{OCH}_{2} \mathrm{CH}_{3}\right), 2.43$ $\left(3 \mathrm{H}, \mathrm{s}, \mathrm{NTsC} H_{3}\right), 3.85\left(3 \mathrm{H}, \mathrm{s}, \mathrm{ArOCH}_{3}\right), 4.29(2 \mathrm{H}, \mathrm{q}, J 6.9$, $\left.\mathrm{OCH}_{2} \mathrm{CH}_{3}\right), 6.18(1 \mathrm{H}, \mathrm{d}, J 16.2, \mathrm{C}(2) H), 6.90(2 \mathrm{H}, \mathrm{d}, J 8.2$, $\left.\mathrm{NSO}_{2} \mathrm{Ar} H\right), 7.32(2 \mathrm{H}, \mathrm{d}, J$ 7.5, C(4) $\mathrm{Ar} H), 7.75(2 \mathrm{H}, \mathrm{d}, J$ 7.0, $\left.\mathrm{NSO}_{2} \mathrm{Ar} H\right), 7.89(2 \mathrm{H}, \mathrm{d}, J$ 7.4, C(4)ArH), 8.01-8.21 (1H, m, C(3)H). Data consistent with literature. ${ }^{13 a}$

\section{Preparation of Dihydropyridinones}

(3S,4S)-Ethyl 2-oxo-3,6-diphenyl-1-(phenylsulfonyl)-1,2,3,4tetrahydropyridine-4-carboxylate 8

Following general procedure $\mathrm{B}$, the reaction of phenylacetic acid (1.36 g, $10.0 \mathrm{mmol}), i$ - $\mathrm{Pr}_{2} \mathrm{NEt}(1.73 \mathrm{~mL}, 10.0 \mathrm{mmol})$ and pivaloyl chloride $(1.23 \mathrm{~mL}, 10.0 \mathrm{mmol})$ in $\mathrm{CH}_{2} \mathrm{Cl}_{2}(25 \mathrm{~mL})$, followed by (-)tetramisole $\cdot \mathrm{HCl} 4(0.24 \mathrm{~g}, 1.0 \mathrm{mmol}, 20 \mathrm{~mol} \%)$, ketimine 6 (1.72 g, $5.0 \mathrm{mmol}), i-\mathrm{Pr}_{2} \mathrm{NEt}(0.87 \mathrm{~mL}, 5.0 \mathrm{mmol})$ and $\mathrm{CH}_{2} \mathrm{Cl}_{2}(50 \mathrm{~mL})$, for 1 $\mathrm{h}$ gave a crude residue of $92: 8 \mathrm{dr}$. Purification by flash chromatography $\left(\mathrm{Et}_{2} \mathrm{O} /\right.$ pet. ether, 1:4) gave 8 (1.48 g, $3.2 \mathrm{mmol}$, $64 \%, 92: 8 \mathrm{dr}$ ) as a white solid; $\mathrm{mp} 128-129^{\circ} \mathrm{C}$; $v_{\max }$ (ATR): 2970 , 1722, 1448, 1155, 989; $[\alpha]_{\mathrm{D}}^{20}+32.9$ (c $\left.0.55, \mathrm{CHCl}_{3}\right)$; HPLC (Chiralpak IA, 20\% IPA / hexane, $0.5 \mathrm{~mL} / \mathrm{min}, 254 \mathrm{~nm}, 30{ }^{\circ} \mathrm{C}$ ): $t_{\mathrm{R}}$ $(3 S, 4 S): 13.2 \mathrm{~min}, t_{\mathrm{R}}(3 R, 4 R): 33.1 \mathrm{~min}, 98 \%$ ee; ${ }^{1} \mathrm{H}$ NMR $(500$ $\left.\mathrm{MHz}, \mathrm{CDCl}_{3}\right) \delta_{\mathrm{H}}: 1.04\left(3 \mathrm{H}, \mathrm{t}, J\right.$ 7.1, $\left.\mathrm{OCH}_{2} \mathrm{CH}_{3}\right), 3.75(1 \mathrm{H}, \mathrm{dd}, J$ 9.6, $4.8, \mathrm{C}(4) H), 4.02\left(2 \mathrm{H}, \mathrm{qd}, J 7.5,3.4, \mathrm{OCH}_{2} \mathrm{CH}_{3}\right), 4.10(1 \mathrm{H}, \mathrm{d}, J 9.6$, $\mathrm{C}(3) H), 5.87(1 \mathrm{H}, \mathrm{d}, J 4.8, \mathrm{C}(5) H), 7.01(2 \mathrm{H}, \mathrm{dd}, J$ 6.4, 2.4, $\mathrm{C}(3) \mathrm{Ph} H), 7.26-7.27 \quad(3 \mathrm{H}, \quad \mathrm{m}, \mathrm{C}(3) \mathrm{Ph} H), 7.38-7.40 \quad(5 \mathrm{H}, \quad \mathrm{m}$, $\mathrm{C}(6) \mathrm{Ph} H), 7.50\left(2 \mathrm{H}, \mathrm{t}, J 7.9, \mathrm{NSO}_{2} \mathrm{Ph} H\right), 7.65(1 \mathrm{H}, \mathrm{t}, J 7.4$, $\left.\mathrm{NSO}_{2} \mathrm{Ph} H\right), 7.95\left(2 \mathrm{H}, \mathrm{d}, J 8.0, \mathrm{NSO}_{2} \mathrm{Ph} H\right) ;{ }^{13} \mathrm{C}\left\{{ }^{1} \mathrm{H}\right\}$ NMR $(126$
$\left.\mathrm{MHz}, \mathrm{CDCl}_{3}\right) \delta_{\mathrm{C}}: 13.9,45.1,54.0,61.6,115.9,126.1,128.5,128.6$, $128.7,128.9,129.5,129.6,132.3,134.1,136.7,137.9,139.2,141.3$, 170.9, 172.2; HRMS $\left(\mathrm{ESI}^{+}\right): \mathrm{C}_{26} \mathrm{H}_{24} \mathrm{NO}_{5} \mathrm{~S}[\mathrm{M}+\mathrm{H}]^{+}$found 462.1373, requires $462.1370(+0.7 \mathrm{ppm})$.

Ethyl 6-oxo-4,5-diphenyl-1-tosyl-1,4,5,6-tetrahydropyridine-2carboxylate 10

Following general procedure $\mathrm{B}$, the reaction of phenylacetic acid (54.5 $\mathrm{mg}, 0.4 \mathrm{mmol}), i$ - $\mathrm{Pr}_{2} \mathrm{NEt}(70 \mu \mathrm{L}, 0.4 \mathrm{mmol})$ and pivaloyl chloride $(50 \mu \mathrm{L}, 0.4 \mathrm{mmol})$ in $\mathrm{CH}_{2} \mathrm{Cl}_{2}(1.0 \mathrm{~mL})$, followed by DHPB 7 (7.6 mg, $0.04 \mathrm{mmol}, 20 \mathrm{~mol} \%$ ), ketimine 9 (71.6 mg, $0.2 \mathrm{mmol}), i$ $\mathrm{Pr}_{2} \mathrm{NEt}(35 \mu \mathrm{L}, 0.2 \mathrm{mmol})$ and $\mathrm{CH}_{2} \mathrm{Cl}_{2}(2.0 \mathrm{~mL})$, for $1 \mathrm{~h}$ gave a crude residue of 59:41 dr. Purification by flash chromatography $\left(\mathrm{Et}_{2} \mathrm{O} /\right.$ petrol, 1:4) gave $\mathbf{1 0}(37.9 \mathrm{mg}, 0.08 \mathrm{mmol}, 40 \%, 54: 46 \mathrm{dr})$ as a white solid; $\mathrm{mp} 118-120^{\circ} \mathrm{C}$; $v_{\max }$ (ATR): 2987, 1724, 1597, 1363, 1170, 1085,$1028 ;{ }^{1} \mathrm{H}$ NMR $\left(300 \mathrm{MHz}, \mathrm{CDCl}_{3}\right.$, both diastereoisomers $) \delta_{\mathrm{H}}$ : 1.37-1.43 $\left(3 \mathrm{H}, \mathrm{m}, \mathrm{OCH}_{2} \mathrm{CH}_{3}\right), 2.44\left(3 \mathrm{H}, \mathrm{s}, \mathrm{NTsCH}_{3}\right), 3.85(1 \mathrm{H}, \mathrm{d}, J$ $10.2, \mathrm{CH}), 4.03(1 \mathrm{H}, \mathrm{d}, J 5.8, \mathrm{CH}), 4.08(2 \mathrm{H}, \mathrm{q}, J$ 5.3, 4.5, $\left.\mathrm{OCH}_{2} \mathrm{CH}_{3}\right), 4.28-4.52\left(3 \mathrm{H}, \mathrm{m}, \mathrm{OCH}_{2} \mathrm{CH}_{3}\right.$ and $\left.\mathrm{CH}\right), 6.54-6.63(1 \mathrm{H}$, $\mathrm{m}, \operatorname{Ar} H), 6.65(1 \mathrm{H}, \mathrm{d}, J 4.2, \operatorname{Ar} H), 6.69(1 \mathrm{H}, \mathrm{d}, J 6.0, \operatorname{Ar} H), 6.85$ $(1 \mathrm{H}, \mathrm{dd} J 7.9,1.5, \mathrm{Ar} H), 6.87-6.94(1 \mathrm{H}, \mathrm{m}, \mathrm{Ar} H), 7.00(1 \mathrm{H}, \mathrm{dd}, J$ 7.4, 5.8, $\operatorname{Ar} H), 7.04-7.13(2 \mathrm{H}, \mathrm{m}, \operatorname{Ar} H), 7.12-7.25(7 \mathrm{H}, \mathrm{m}, \mathrm{Ar} H)$, $7.32(2 \mathrm{H}, \mathrm{t}, J$ 8.2, $\mathrm{Ar} H), 8.11(2 \mathrm{H}, \mathrm{d}, J$ 8.4 $\mathrm{Ar} H), 8.14-8.23(2 \mathrm{H}, \mathrm{m}$, $\mathrm{Ar} H) ;{ }^{13} \mathrm{C}\left\{{ }^{1} \mathrm{H}\right\}$ NMR $\left(101 \mathrm{MHz}, \mathrm{CD}_{2} \mathrm{Cl}_{2}\right) \delta_{\mathrm{C}}: 13.9,21.5,44.1,44.5$, 55.9, 57.2, 62.2, 126.9, 127.5, 127.6, 127.7, 127.8, 127.9, 127.9, $128.5,128.5,128.8,128.8,128.9,129.3,129.3,129.4,129.5$ 129.6, 129.9, 131.9, 132.8, 133.2, 135.4, 135.7, 138.8, 145.8, 170.2, 170.9; HRMS $\left(\mathrm{ESI}^{+}\right): \mathrm{C}_{27} \mathrm{H}_{26} \mathrm{NO}_{5} \mathrm{~S}[\mathrm{M}+\mathrm{H}]^{+}$found 476.1527, requires $476.1526(+0.2 \mathrm{ppm})$.

(3S,4S)-Ethyl tetrahydropyridine-4-carboxylate 18

Following general procedure $\mathrm{B}$, the reaction of phenylacetic acid (1.36 g, $10.0 \mathrm{mmol}), i$ - $\mathrm{Pr}_{2} \mathrm{NEt}(1.73 \mathrm{~mL}, 10.0 \mathrm{mmol})$ and pivaloyl chloride (1.23 mL, $10.0 \mathrm{mmol})$ in $\mathrm{CH}_{2} \mathrm{Cl}_{2}(25 \mathrm{~mL})$, followed by (-)tetramisole $\cdot \mathrm{HCl} 4(0.24 \mathrm{~g}, 1.0 \mathrm{mmol}, 20 \mathrm{~mol} \%)$, ketimine 14a (1.79 g, $5.0 \mathrm{mmol}), i-\mathrm{Pr}_{2} \mathrm{NEt}(0.87 \mathrm{~mL}, 5.0 \mathrm{mmol})$ and $\mathrm{CH}_{2} \mathrm{Cl}_{2}(50 \mathrm{~mL})$, for $1 \mathrm{~h}$ gave a crude residue of 92:8 dr. Purification by flash chromatography $\left(\mathrm{Et}_{2} \mathrm{O} /\right.$ pet. ether, 1:4) gave 18 (1.47 g, $3.1 \mathrm{mmol}$, $62 \%, 93: 7 \mathrm{dr})$ as a white solid. Further recrystallisation $\left(\mathrm{Et}_{2} \mathrm{O}\right)$ gave 18 as a single diastereoisomer $(0.80 \mathrm{~g}, 1.68 \mathrm{mmol}, 33 \%)$ as a white solid; mp 129-130 ${ }^{\circ} \mathrm{C} ; v_{\max }$ (ATR): 2978, 1726, 1363, 1166, 927; $[\alpha]_{\mathrm{D}}^{20}+50.2\left(c \quad 0.49, \mathrm{CHCl}_{3}\right)$; HPLC (Chiralpak IA, 30\% IPA / hexane, $\left.0.5 \mathrm{~mL} / \mathrm{min}, 254 \mathrm{~nm}, 30{ }^{\circ} \mathrm{C}\right): t_{\mathrm{R}}(3 S, 4 S): 20.0 \mathrm{~min}, t_{\mathrm{R}}(3 R$, $4 R): 40.6 \mathrm{~min},>99 \%$ ee; ${ }^{1} \mathrm{H}$ NMR $\left(500 \mathrm{MHz}, \mathrm{CDCl}_{3}\right) \delta_{\mathrm{H}}: 1.08(3 \mathrm{H}$, t, $J$ 7.1, $\left.\mathrm{OCH}_{2} \mathrm{CH}_{3}\right), 2.49\left(3 \mathrm{H}, \mathrm{s}, \mathrm{TsCH} \mathrm{S}_{3}\right), 3.79(1 \mathrm{H}, \mathrm{dd}, J$ 9.6, 4.8, $\mathrm{C}(4) H), 4.05\left(2 \mathrm{H}, \mathrm{qd}, J\right.$ 7.0, 3.7, $\left.\mathrm{OCH}_{2} \mathrm{CH}_{3}\right), 4.13(1 \mathrm{H}, \mathrm{d}, J 9.6$, $\mathrm{C}(3) H), 5.89(1 \mathrm{H}, \mathrm{d}, J 4.8, \mathrm{C}(5) H), 7.06(2 \mathrm{H}, \mathrm{dd}, J 6.9,2.2$, $\mathrm{C}(3) \mathrm{Ph} H), 7.30-7.33(8 \mathrm{H}, \mathrm{m}, \mathrm{Ph} H), 7.40-7.45(2 \mathrm{H}, \mathrm{d}, J 9.0, \mathrm{NTs} H)$, $7.87(2 \mathrm{H}, \mathrm{d}, J$ 9.0, NTs $H) ;{ }^{13} \mathrm{C}\left\{{ }^{1} \mathrm{H}\right\}$ NMR $\left(101 \mathrm{MHz}, \mathrm{CDCl}_{3}\right) \delta_{\mathrm{C}}$ : 14.0, 21.9, 45.3, 54.1, 61.7, 115.8, 126.2, 128.1, 128.6, 128.7, 128.8, 128.9, 129.3, 129.6, 135.5, 136.4, 136.8, 141.4, 145.3, 170.9, 172.0; HRMS (ESI+): $\mathrm{C}_{29} \mathrm{H}_{26} \mathrm{NO}_{5} \mathrm{~S}[\mathrm{M}+\mathrm{H}]^{+}$found 476.1526, requires $476.1526( \pm 0.0 \mathrm{ppm})$.

$(3 S, 4 S)$-Ethyl 1-((4-methoxyphenyl)sulfonyl)-2-oxo-3,6-diphenyl1,2,3,4-tetrahydropyridine-4-carboxylate 19

Following general procedure $\mathrm{B}$, the reaction of phenylacetic acid (54.5 mg, $0.4 \mathrm{mmol}), i$ - $\mathrm{Pr}_{2} \mathrm{NEt}(70 \mu \mathrm{L}, 0.4 \mathrm{mmol})$ and pivaloyl chloride $(50 \mu \mathrm{L}, 0.4 \mathrm{mmol})$ in $\mathrm{CH}_{2} \mathrm{Cl}_{2}(1.0 \mathrm{~mL})$, followed by (-)tetramisole $\cdot \mathrm{HCl} 4(9.6 \mathrm{mg}, 0.04 \mathrm{mmol}, 20 \mathrm{~mol} \%)$, ketimine 14b (68.7 mg, $0.2 \mathrm{mmol}), i-\mathrm{Pr}_{2} \mathrm{NEt}(35 \mu \mathrm{L}, 0.2 \mathrm{mmol})$ and $\mathrm{CH}_{2} \mathrm{Cl}_{2}(2.0$ 
$\mathrm{mL}$ ), for $1 \mathrm{~h}$ gave a crude residue of 95:5 dr. Purification by flash chromatography $\left(\mathrm{Et}_{2} \mathrm{O} /\right.$ pet. ether, 1:4) gave $19(58.2 \mathrm{mg}, 0.12$ mmol, $59 \%, 90: 10 \mathrm{dr}$ ) as a white solid; $\mathrm{mp} 114-115^{\circ} \mathrm{C} ; v_{\max }$ (ATR): 2976, 1720, 1593, 1531, 1496, 1446, 1365, 1303, 1261, 1161, 1087, 1024, 979; $[\alpha]_{\mathrm{D}}^{20}+18.1\left(c \quad 0.52, \mathrm{CHCl}_{3}\right)$; HPLC (Chiralpak AD-H, $30 \% \mathrm{IPA} /$ hexane, $\left.1.0 \mathrm{~mL} / \mathrm{min}, 254 \mathrm{~nm}, 30^{\circ} \mathrm{C}\right): t_{\mathrm{R}}(3 S, 4 S): 17.5$ $\min , t_{\mathrm{R}}(3 R, 4 R): 55.6 \mathrm{~min}, 99 \%$ ee; ${ }^{1} \mathrm{H} \mathrm{NMR}\left(400 \mathrm{MHz}, \mathrm{CDCl}_{3}\right) \delta_{\mathrm{H}}$ : $1.04\left(3 \mathrm{H}, \mathrm{t}, J 7.1, \mathrm{OCH}_{2} \mathrm{CH}_{3}\right), 3.74(1 \mathrm{H}, \mathrm{dd}, J 9.7,4.8, \mathrm{C}(4) H), 3.89$ $\left(3 \mathrm{H}, \mathrm{s}, \mathrm{ArOCH}_{3}\right), 4.01\left(2 \mathrm{H}, \mathrm{qd}, J 7.1,1.7, \mathrm{OCH}_{2} \mathrm{CH}_{3}\right), 4.08(1 \mathrm{H}, \mathrm{d}, J$ 9.7, C(3)H), $5.83(1 \mathrm{H}, \mathrm{d}, J 4.8, \mathrm{C}(5) H), 6.93(2 \mathrm{H}, \mathrm{d}, J$ 9.1, $\left.\mathrm{NSO}_{2} \mathrm{Ar} H\right), 7.01-7.07 \quad(2 \mathrm{H}, \quad \mathrm{m}, \mathrm{C}(3) \mathrm{Ph} H), 7.26-7.30 \quad(3 \mathrm{H}, \quad \mathrm{m}$, $\mathrm{C}(3) \mathrm{Ph} H), 7.34-7.42 \quad(5 \mathrm{H}, \mathrm{m}, \mathrm{C}(6) \mathrm{Ph} H), 7.87 \quad(2 \mathrm{H}, \mathrm{d}, J$ 9.1, $\left.\mathrm{NSO}_{2} \mathrm{Ar} H\right) ;{ }^{13} \mathrm{C}\left\{{ }^{1} \mathrm{H}\right\}$ NMR $\left(126 \mathrm{MHz}, \mathrm{CDCl}_{3}\right) \delta_{\mathrm{C}}: 14.0,45.2,54.1$, 55.9, 61.7, 113.7, 115.7, 126.1, 128.1, 128.5, 128.7, 128.9, 129.8, $130.5,131.9,135.5,136.8,141.3,164.1,170.9,172.0$; HRMS (ESI+): $\mathrm{C}_{27} \mathrm{H}_{26} \mathrm{NO}_{6} \mathrm{~S}[\mathrm{M}+\mathrm{H}]^{+}$found 492.1470 , requires 492.1475 $(-1.1 \mathrm{ppm})$.

(3S,4S)-Ethyl 1-((4-nitrophenyl)sulfonyl)-2-oxo-3,6-diphenyl1,2,3,4-tetrahydropyridine-4-carboxylate 20

Following general procedure $\mathrm{B}$, the reaction of phenylacetic acid (54.5 mg, $0.4 \mathrm{mmol}), i-\mathrm{Pr}_{2} \mathrm{NEt}(70 \mu \mathrm{L}, 0.4 \mathrm{mmol})$ and pivaloyl chloride $(50 \mu \mathrm{L}, 0.4 \mathrm{mmol})$ in $\mathrm{CH}_{2} \mathrm{Cl}_{2}(1.0 \mathrm{~mL})$, followed by (-)tetramisole $\cdot \mathrm{HCl} 4(9.6 \mathrm{mg}, 0.04 \mathrm{mmol}, 20 \mathrm{~mol} \%)$, ketimine $14 \mathrm{c}$ (68.7 mg, $0.2 \mathrm{mmol}), i$ - $\mathrm{Pr}_{2} \mathrm{NEt}(35 \mu \mathrm{L}, 0.2 \mathrm{mmol})$ and $\mathrm{CH}_{2} \mathrm{Cl}_{2}(2.0$ $\mathrm{mL}$ ), for $1 \mathrm{~h}$ gave a crude residue of 95:5 dr. Purification by flash chromatography $\left(\mathrm{Et}_{2} \mathrm{O} /\right.$ pet. ether, 1:4) gave $20(67 \mathrm{mg}, 0.13 \mathrm{mmol}$, $66 \%,>95: 5 \mathrm{dr}$ ) as a white solid; $\mathrm{mp} 178-180{ }^{\circ} \mathrm{C}$; $v_{\max }$ (ATR): 3066 , 1728, 1521, 1373, 1348, 1261, 1172, 1147, 1107, 1083, 1026, 931; $[\alpha]_{\mathrm{D}}^{20}+4.5\left(c \quad 0.65, \mathrm{CHCl}_{3}\right)$; HPLC (Chiralpak IA, 30\% IPA / hexane, $\left.1.0 \mathrm{~mL} / \mathrm{min}, 254 \mathrm{~nm}, 30^{\circ} \mathrm{C}\right): t_{\mathrm{R}}(3 S, 4 S): 14.8 \mathrm{~min}, t_{\mathrm{R}}(3 R$, 4R): $25.4 \mathrm{~min}, 98 \%$ ee; ${ }^{1} \mathrm{H}$ NMR $\left(500 \mathrm{MHz}, \mathrm{CDCl}_{3}\right) \delta_{\mathrm{H}}: 1.12(3 \mathrm{H}, \mathrm{t}$, $J$ 7.1, $\left.\mathrm{OCH}_{2} \mathrm{CH}_{3}\right), 3.79(1 \mathrm{H}, \mathrm{dd}, J 7.8,5.6, \mathrm{C}(4) H), 4.07(2 \mathrm{H}, \mathrm{qd}, J$ 7.8, 2.8, $\left.\mathrm{OCH}_{2} \mathrm{CH}_{3}\right), 4.15(1 \mathrm{H}, \mathrm{d}, J 5.6, \mathrm{C}(3) H), 5.84(1 \mathrm{H}, \mathrm{d}, J 5.6$, $\mathrm{C}(5) H), 7.13(1 \mathrm{H}, \mathrm{dd}, J 7.5,1.8, \mathrm{C}(3) \mathrm{Ph} H), 7.28-7.44(8 \mathrm{H}, \mathrm{m}, \mathrm{Ph} H)$, $8.10\left(2 \mathrm{H}, \mathrm{d}, J\right.$ 9.0, $\left.\mathrm{NSO}_{2} \mathrm{Ar} H\right), 8.30\left(2 \mathrm{H}, \mathrm{d}, J\right.$ 9.0, $\left.\mathrm{NSO}_{2} \mathrm{Ar} H\right)$; ${ }^{13} \mathrm{C}\left\{{ }^{1} \mathrm{H}\right\}$ NMR $\left(101 \mathrm{MHz}, \mathrm{CDCl}_{3}\right) \delta_{\mathrm{C}}: 14.1,44.9,53.7,62.0,116.2$, 123.7, 126.3, 128.4, 128.4, 128.7, 129.1, 129.3, 131.0, 135.0, 136.2, 141.1, 144.4, 150.8, 170.7, 172.0; HRMS (ESI+): $\mathrm{C}_{26} \mathrm{H}_{23} \mathrm{~N}_{2} \mathrm{O}_{7} \mathrm{~S}$ $[\mathrm{M}+\mathrm{H}]^{+}$found 507.1213 , requires $507.1220(-1.5 \mathrm{ppm})$.

$(3 S, 4 S)$-Ethyl

2-oxo-3,6-diphenyl-1-((2,4,6triisopropylphenyl)sulfonyl)-1,2,3,4-tetrahydropyridine-4carboxylate 21

Following general procedure $\mathrm{B}$, the reaction of phenylacetic acid (54.5 mg, $0.4 \mathrm{mmol}), i$ - $\operatorname{Pr}_{2} \mathrm{NEt}(70 \mu \mathrm{L}, 0.4 \mathrm{mmol})$ and pivaloyl chloride $(50 \mu \mathrm{L}, 0.4 \mathrm{mmol})$ in $\mathrm{CH}_{2} \mathrm{Cl}_{2}(1.0 \mathrm{~mL})$, followed by (-)tetramisole $\cdot \mathrm{HCl} 4(9.6 \mathrm{mg}, 0.04 \mathrm{mmol}, 20 \mathrm{~mol} \%)$, ketimine 14d (93.9 mg, $0.2 \mathrm{mmol}), i$ - $\mathrm{Pr}_{2} \mathrm{NEt}(35 \mu \mathrm{L}, 0.2 \mathrm{mmol})$ and $\mathrm{CH}_{2} \mathrm{Cl}_{2}(2.0$ $\mathrm{mL}$ ), for $1 \mathrm{~h}$ gave a crude residue of $>95: 5 \mathrm{dr}$. Recrystallisation (pet. ether) gave $21(15.4 \mathrm{mg}, 0.03 \mathrm{mmol}, 13 \%,>95: 5 \mathrm{dr})$ as a white solid; mp 215-216 ${ }^{\circ} \mathrm{C} ; v_{\max }$ (ATR): 2960, 1734, 1715, 1599, 1172, 938; $[\alpha]_{\mathrm{D}}^{20}+110\left(c 0.15, \mathrm{CH}_{2} \mathrm{Cl}_{2}\right)$; HPLC (Chiralpak AD-H, 5\% IPA / hexane, $\left.1.5 \mathrm{~mL} / \mathrm{min}, 254 \mathrm{~nm}, 40{ }^{\circ} \mathrm{C}\right): t_{\mathrm{R}}(3 S, 4 S): 3.44 \mathrm{~min}, t_{\mathrm{R}}(3 R$, $4 R): 3.66 \mathrm{~min}, 93 \%$ ee; ${ }^{1} \mathrm{H} \mathrm{NMR}\left(500 \mathrm{MHz}, \mathrm{CDCl}_{3}\right) \delta_{\mathrm{H}}: 0.96(3 \mathrm{H}, \mathrm{t}$, $J$ 7.1, $\left.\mathrm{OCH}_{2} \mathrm{CH}_{3}\right), 1.27\left(6 \mathrm{H}, \mathrm{d}, J 6.5, \mathrm{NSO}_{2} \mathrm{ArCH}\left(\mathrm{CH}_{3}\right)_{2}\right), 1.29-1.37$ $\left(12 \mathrm{H}, \quad \mathrm{m}, \quad \mathrm{NSO}_{2} \mathrm{ArCH}\left(\mathrm{CH}_{3}\right)_{2}\right), \quad 2.96 \quad(1 \mathrm{H}, \quad$ septet, $J \quad 6.9$, $\left.\mathrm{NSO}_{2} \mathrm{ArCH}\left(\mathrm{CH}_{3}\right)_{2}\right), 3.72(1 \mathrm{H}, \mathrm{dd}, J 12.9,3.4, \mathrm{C}(4) H), 3.95(2 \mathrm{H}, \mathrm{q}, J$ 7.1, $\left.\mathrm{OCH}_{2} \mathrm{CH}_{3}\right), 4.04(1 \mathrm{H}, \mathrm{d}, J 12.9, \mathrm{C}(3) H), 4.18(2 \mathrm{H}, \mathrm{d}, J 6.6$, $\left.\mathrm{NSO}_{2} \mathrm{ArCH}\left(\mathrm{CH}_{3}\right)_{2}\right), 5.80(1 \mathrm{H}, \mathrm{d}, J 3.4, \mathrm{C}(5) H), 6.71-6.84(2 \mathrm{H}, \mathrm{m}$, $\mathrm{PhH}$ ), 7.17-7.23 (5H, m, $\mathrm{PhH}$ and $\left.\mathrm{NSO}_{2} \mathrm{Ar} H\right)$, 7.39-7.47 (3H, m,
$\mathrm{Ph} H$ ), 7.61-7.63 (2H, m, $\mathrm{Ph} H) ;{ }^{13} \mathrm{C}\left\{{ }^{1} \mathrm{H}\right\}$ NMR $\left(101 \mathrm{MHz}, \mathrm{CDCl}_{3}\right)$ $\delta_{\mathrm{C}}: 13.9,23.7,23.8,25.0,25.4,29.9,34.4,45.9,53.3,61.5,114.8$, $123.8,126.2,127.8,128.4,128.6,129.0,129.2,133.7,135.5,137.1$, 142.1, 152.4, 154.2, 171.0, 171.2; HRMS (ESI+): $\mathrm{C}_{35} \mathrm{H}_{41} \mathrm{NO}_{5} \mathrm{SNa}$ $[\mathrm{M}+\mathrm{Na}]^{+}$found 610.2592 , requires $610.2603(-1.2 \mathrm{ppm})$.

(3S,4S)-Ethyl 1-(methylsulfonyl)-2-oxo-3,6-diphenyl-1,2,3,4tetrahydropyridine-4-carboxylate 22

Following general procedure $\mathrm{B}$, the reaction of phenylacetic acid (54.5 mg, $0.4 \mathrm{mmol}), i$ - $\mathrm{Pr}_{2} \mathrm{NEt}(70 \mu \mathrm{L}, 0.4 \mathrm{mmol})$ and pivaloyl chloride $(50 \mu \mathrm{L}, 0.4 \mathrm{mmol})$ in $\mathrm{CH}_{2} \mathrm{Cl}_{2}(1.0 \mathrm{~mL})$, followed by (-)tetramisole $\cdot \mathrm{HCl} 4(9.6 \mathrm{mg}, 0.04 \mathrm{mmol}, 20 \mathrm{~mol} \%)$, ketimine $14 \mathrm{e}$ (68.7 mg, $0.2 \mathrm{mmol}), i-\mathrm{Pr}_{2} \mathrm{NEt}(35 \mu \mathrm{L}, 0.2 \mathrm{mmol})$ and $\mathrm{CH}_{2} \mathrm{Cl}_{2}(2.0$ $\mathrm{mL}$ ), for $1 \mathrm{~h}$ gave a crude residue of 91:9 dr. Purification by flash chromatography ( $\mathrm{Et}_{2} \mathrm{O} /$ pet. ether, 1:4) gave 22 (47.1 mg, 0.12 mmol, $59 \%, 92: 8 \mathrm{dr}$ ) as a white solid; $\mathrm{mp} 131-132{ }^{\circ} \mathrm{C}$; $v_{\max }$ (ATR): $2916,1708,1653,1496,1446,1344,1165,1151,1118,960 ;[\alpha]_{D}^{20}$ -6.3 (c $0.51, \mathrm{CHCl}_{3}$ ); HPLC (Chiralpak IA, 30\% IPA / hexane, 0.5 $\left.\mathrm{mL} / \mathrm{min}, 254 \mathrm{~nm}, 30{ }^{\circ} \mathrm{C}\right): t_{\mathrm{R}}(3 S, 4 S): 41.5 \mathrm{~min}, t_{\mathrm{R}}(3 R, 4 R): 45.0$ min, 99\% ee; ${ }^{1} \mathrm{H}$ NMR $\left(500 \mathrm{MHz}, \mathrm{CDCl}_{3}\right) \delta_{\mathrm{H}}: 1.18(1 \mathrm{H}, \mathrm{t}, J$ 7.1, $\left.\mathrm{OCH}_{2} \mathrm{CH}_{3}\right), 3.46\left(3 \mathrm{H}, \mathrm{s}, \mathrm{MsCH}_{3}\right), 3.78(1 \mathrm{H}, \mathrm{t}, J 6.3, \mathrm{C}(4) H), 4.14$ $\left(2 \mathrm{H}, \mathrm{q}, J 7.1, \mathrm{OCH}_{2} \mathrm{CH}_{3}\right), 4.26(1 \mathrm{H}, \mathrm{d}, J 6.3, \mathrm{C}(3) H), 5.76(1 \mathrm{H}, \mathrm{d}, J$ 6.2, $\mathrm{C}(5) H), 7.20-7.48(10 \mathrm{H}, \mathrm{m}, \mathrm{PhH}) ;{ }^{13} \mathrm{C}\left\{{ }^{1} \mathrm{H}\right\}$ NMR (101 MHz, $\left.\mathrm{CDCl}_{3}\right) \delta_{\mathrm{C}}: 14.1,43.9,44.9,53.2,62.0,114.3,125.5,128.2,128.3$, $128.8,129.9,129.1,135.0,137.0,141.2,171.2,173.0$; HRMS (ESI+): $\mathrm{C}_{21} \mathrm{H}_{22} \mathrm{NO}_{5} \mathrm{~S}[\mathrm{M}+\mathrm{H}]^{+}$found 400.1216 , requires 400.1213 $(+0.7 \mathrm{ppm})$.

$(3 S, 4 S)$-Ethyl 2-oxo-6-phenyl-1-(phenylsulfonyl)-3-(p-tolyl)1,2,3,4-tetrahydropyridine-4-carboxylate 23

Following general procedure $\mathrm{B}$, the reaction of $p$-tolylacetic acid (60.1 mg, $0.4 \mathrm{mmol}), i$ - $\mathrm{Pr}_{2} \mathrm{NEt}(70 \mu \mathrm{L}, 0.4 \mathrm{mmol})$ and pivaloyl chloride $(50 \mu \mathrm{L}, 0.4 \mathrm{mmol})$ in $\mathrm{CH}_{2} \mathrm{Cl}_{2}(1.0 \mathrm{~mL})$, followed by (-)tetramisole $\cdot \mathrm{HCl} 4(9.6 \mathrm{mg}, 0.04 \mathrm{mmol}, 20 \mathrm{~mol} \%)$, ketimine 6 (68.7 $\mathrm{mg}, 0.2 \mathrm{mmol}), i-\mathrm{Pr}_{2} \mathrm{NEt}(35 \mu \mathrm{L}, 0.2 \mathrm{mmol})$ and $\mathrm{CH}_{2} \mathrm{Cl}_{2}(2.0 \mathrm{~mL})$, for $1 \mathrm{~h}$ gave a crude residue of 92:8 dr. Purification by flash chromatography $\left(\mathrm{Et}_{2} \mathrm{O} /\right.$ pet. ether, 1:4) gave 23 (63.2 mg, 0.13 mmol, $66 \%, 94: 6 \mathrm{dr}$ ) as a white solid; $\mathrm{mp} 100-102{ }^{\circ} \mathrm{C} ; v_{\max }$ (ATR): 2933, 1730, 1516, 1355, 1159, 929; $[\alpha]_{\mathrm{D}}^{20}+31.1\left(c 0.54, \mathrm{CH}_{2} \mathrm{Cl}_{2}\right)$; HPLC (Chiralpak IA, 20\% IPA / hexane, $0.5 \mathrm{~mL} / \mathrm{min}, 254 \mathrm{~nm}, 30$ $\left.{ }^{\circ} \mathrm{C}\right): t_{\mathrm{R}}(3 S, 4 S): 30.4 \mathrm{~min}, t_{\mathrm{R}}(3 R, 4 R): 55.2 \mathrm{~min}, 98 \%$ ee; ${ }^{1} \mathrm{H}$ NMR $\left(400 \mathrm{MHz}, \mathrm{CDCl}_{3}\right) \delta_{\mathrm{H}}: 1.06\left(3 \mathrm{H}, \mathrm{t}, J 7.1, \mathrm{OCH}_{2} \mathrm{CH}_{3}\right), 2.29(3 \mathrm{H}, \mathrm{s}, p-$ tolylArCH$\left.H_{3}\right), 3.72(1 \mathrm{H}, \mathrm{dd}, J 9.7,4.8, \mathrm{C}(4) H), 3.97-4.07(3 \mathrm{H}, \mathrm{m}$, $\mathrm{C}(3) \mathrm{H}$ and $\left.\mathrm{OCH}_{2} \mathrm{CH}_{3}\right), 5.84(1 \mathrm{H}, \mathrm{d}, J 4.8, \mathrm{C}(5) H), 6.88(2 \mathrm{H}, \mathrm{d}, J 8.1$, $\mathrm{C}(3) \mathrm{Ar} H), 7.07 \quad(2 \mathrm{H}, \mathrm{d}, J$ 7.9, $\mathrm{C}(3) \mathrm{Ar} H), 7.31-7.42 \quad(5 \mathrm{H}, \mathrm{m}$, $\mathrm{C}(6) \mathrm{Ph} H), 7.49\left(2 \mathrm{H}, \mathrm{t}, J\right.$ 7.4, $\left.\mathrm{NSO}_{2} \mathrm{Ph} H\right), 7.64(1 \mathrm{H}, \mathrm{t}, J$ 7.4, $\left.\mathrm{NSO}_{2} \mathrm{Ph} H\right), 7.94\left(2 \mathrm{H}, \mathrm{d}, J 1.5, \mathrm{NSO}_{2} \mathrm{Ph} H\right) ;{ }^{13} \mathrm{C}\left\{{ }^{1} \mathrm{H}\right\}$ NMR $(126$ $\left.\mathrm{MHz}, \mathrm{CDCl}_{3}\right) \delta_{\mathrm{C}}: 14.2,21.2,45.2,53.7,61.7,116.0,126.2,128.5$, 128.6, 128.7, 128.9, 129.5, 129.6, 132.3, 134.1, 136.7, 137.9, 139.2, 141.3, 170.9, 172.2; HRMS (ESI+): $\mathrm{C}_{27} \mathrm{H}_{26} \mathrm{NO}_{5} \mathrm{~S}[\mathrm{M}+\mathrm{H}]^{+}$found 476.1524, requires $476.1526(-0.5 \mathrm{ppm})$.

$(3 S, 4 S)$-Ethyl 2-oxo-6-phenyl-1-(phenylsulfonyl)-3-(m-tolyl)1,2,3,4-tetrahydropyridine-4-carboxylate 24

Following general procedure $\mathrm{B}$, the reaction of $m$-tolylacetic acid (60.1 $\mathrm{mg}, 0.4 \mathrm{mmol}), i$ - $\mathrm{Pr}_{2} \mathrm{NEt}(70 \mu \mathrm{L}, 0.4 \mathrm{mmol})$ and pivaloyl chloride $(50 \mu \mathrm{L}, 0.4 \mathrm{mmol})$ in $\mathrm{CH}_{2} \mathrm{Cl}_{2}(1.0 \mathrm{~mL})$, followed by (-)tetramisole $\cdot \mathrm{HCl} 4(9.6 \mathrm{mg}, 0.04 \mathrm{mmol}, 20 \mathrm{~mol} \%)$, ketimine 6 (68.7 $\mathrm{mg}, 0.2 \mathrm{mmol}), i-\mathrm{Pr}_{2} \mathrm{NEt}(35 \mu \mathrm{L}, 0.2 \mathrm{mmol})$ and $\mathrm{CH}_{2} \mathrm{Cl}_{2}(2.0 \mathrm{~mL})$, for $1 \mathrm{~h}$ gave a crude residue of 92:8 dr. Purification by flash chromatography $\left(\mathrm{Et}_{2} \mathrm{O} /\right.$ pet. ether, 1:4) gave 24 (69.6 mg, 0.15 mmol, $72 \%, 93: 7 \mathrm{dr}$ ) as a white solid; $\mathrm{mp} 134-136^{\circ} \mathrm{C} ; v_{\max }$ (ATR): 
2922, 1726, 1516, 1359, 1155, 887; $[\alpha]_{\mathrm{D}}^{20}+27.9\left(c 0.64, \mathrm{CH}_{2} \mathrm{Cl}_{2}\right)$; HPLC (Chiralpak IA, 10\% IPA / hexane, $1.0 \mathrm{~mL} / \mathrm{min}, 254 \mathrm{~nm}, 30$ $\left.{ }^{\circ} \mathrm{C}\right): t_{\mathrm{R}}(3 S, 4 S): 22.7 \mathrm{~min}, t_{\mathrm{R}}(3 R, 4 R): 62.7 \mathrm{~min}, 99 \%$ ee; ${ }^{1} \mathrm{H} \mathrm{NMR}$ $\left(500 \mathrm{MHz}, \mathrm{CDCl}_{3}\right) \delta_{\mathrm{H}}: 1.05\left(3 \mathrm{H}, \mathrm{t}, J 7.1, \mathrm{OCH}_{2} \mathrm{CH}_{3}\right), 2.26(3 \mathrm{H}, \mathrm{s}, m-$ tolylArCH$\left.)_{3}\right), 3.72(1 \mathrm{H}, \mathrm{dd}, J$ 9.5, 4.9, C(4)H), 3.98-4.07 $(3 \mathrm{H}, \mathrm{m}$, $\mathrm{C}(3) \mathrm{H}$ and $\left.\mathrm{OCH}_{2} \mathrm{CH}_{3}\right), 5.84(1 \mathrm{H}, \mathrm{d}, J 4.9, \mathrm{C}(5) H), 6.77-6.81(2 \mathrm{H}, \mathrm{m}$, $\mathrm{C}(3) \operatorname{Ar} H), 7.05(1 \mathrm{H}, \mathrm{d}, J$ 7.6, C(3)ArH), $7.14(1 \mathrm{H}, \mathrm{t}, J$ 7.6, $\mathrm{C}(3) \mathrm{Ar} H), 7.33-7.42 \quad(5 \mathrm{H}, \mathrm{m}, \mathrm{C}(6) \mathrm{Ph} H), 7.50 \quad(2 \mathrm{H}, \mathrm{t}, J$ 7.9, $\left.\mathrm{NSO}_{2} \mathrm{Ph} H\right), 7.65\left(1 \mathrm{H}, \mathrm{t}, J 7.4, \mathrm{NSO}_{2} \mathrm{Ph} H\right), 7.96(2 \mathrm{H}, \mathrm{d}, J 1$, $\left.\mathrm{NSO}_{2} \mathrm{Ph} H\right) ;{ }^{13} \mathrm{C}\left\{{ }^{1} \mathrm{H}\right\}$ NMR $\left(126 \mathrm{MHz}, \mathrm{CDCl}_{3}\right) \delta_{\mathrm{C}}: 14.0,21.5,45.2$, 53.9, 61.7, 115.8, 125.6, 126.1, $128.6(2 \times C), 128.6,128.7,128.9$, 129.3, 129.5, 134.1, 135.3, 136.7, 138.4, 139.2, 141.3, 170.8, 172.0; HRMS (ESI+): $\mathrm{C}_{27} \mathrm{H}_{26} \mathrm{NO}_{5} \mathrm{~S}[\mathrm{M}+\mathrm{H}]^{+}$found 476.1522, requires $476.1526(-0.9 \mathrm{ppm})$.

\section{(3S,4S)-Ethyl 2-oxo-6-phenyl-1-(phenylsulfonyl)-3-(o-tolyl)-} 1,2,3,4-tetrahydropyridine-4-carboxylate 25

Following general procedure $\mathrm{B}$, the reaction of $o$-tolylacetic acid (60.1 mg, $0.4 \mathrm{mmol}), i-\mathrm{Pr}_{2} \mathrm{NEt}(70 \mu \mathrm{L}, 0.4 \mathrm{mmol})$ and pivaloyl chloride $(50 \mu \mathrm{L}, 0.4 \mathrm{mmol})$ in $\mathrm{CH}_{2} \mathrm{Cl}_{2}(1.0 \mathrm{~mL})$, followed by (-)tetramisole $\cdot \mathrm{HCl} 4(9.6 \mathrm{mg}, 0.04 \mathrm{mmol}, 20 \mathrm{~mol} \%)$, ketimine 6 (68.7 $\mathrm{mg}, 0.2 \mathrm{mmol}), i-\mathrm{Pr}_{2} \mathrm{NEt}(35 \mu \mathrm{L}, 0.2 \mathrm{mmol})$ and $\mathrm{CH}_{2} \mathrm{Cl}_{2}(2.0 \mathrm{~mL})$, for $1 \mathrm{~h}$ gave a crude residue of 92:8 dr. Purification by flash chromatography $\left(\mathrm{Et}_{2} \mathrm{O} /\right.$ pet. ether, 1:4) gave 25 (63.9 mg, 0.13 mmol, 67\%, 93:7 dr) as a white solid; $m p$ 78-80 ${ }^{\circ} \mathrm{C} ; v_{\max }$ (ATR): 2922, 1726, 1516, 1359, 1155, 887; $[\alpha]_{\mathrm{D}}^{20}+45.8\left(c 0.55, \mathrm{CH}_{2} \mathrm{Cl}_{2}\right)$; HPLC (Chiralpak IA, 10\% IPA / hexane, $1.0 \mathrm{~mL} / \mathrm{min}, 254 \mathrm{~nm}, 30$ $\left.{ }^{\circ} \mathrm{C}\right): t_{\mathrm{R}}(3 S, 4 S): 16.3 \mathrm{~min}, t_{\mathrm{R}}(3 R, 4 R): 56.5 \mathrm{~min}, 99 \%$ ee; ${ }^{1} \mathrm{H} \mathrm{NMR}$ $\left(400 \mathrm{MHz}, \mathrm{CDCl}_{3}\right) \delta_{\mathrm{H}}: 1.00\left(1 \mathrm{H}, \mathrm{t}, J 7.1, \mathrm{OCH}_{2} \mathrm{CH}_{3}\right), 2.25(3 \mathrm{H}, \mathrm{s}, o-$ tolyl $\left.\mathrm{ArCH}_{3}\right), 3.85(1 \mathrm{H}, \mathrm{dd}, J 10.9,4.3, \mathrm{C}(4) H), 4.00(2 \mathrm{H}, \mathrm{q}, J 7.1$, $\left.\mathrm{OCH}_{2} \mathrm{CH}_{3}\right), 4.31(1 \mathrm{H}, \mathrm{d}, J 10.9, \mathrm{C}(3) H), 5.90(1 \mathrm{H}, \mathrm{d}, J 4.3, \mathrm{C}(5) H)$, $6.80(1 \mathrm{H}, \mathrm{d}, J 7.8, \mathrm{C}(3) \mathrm{Ar} H), 7.09(1 \mathrm{H}, \mathrm{td}, J 7.8, \mathrm{C}(3) \mathrm{Ar} H), 7.12-$ $7.20(2 \mathrm{H}, \mathrm{m}, \mathrm{C}(3) \mathrm{Ar} H$ and $\mathrm{C}(3) \mathrm{Ar} H)$, 7.37-7.48 (5H, m, C(6)PhH), $7.51\left(2 \mathrm{H}, \mathrm{td}, J 7.6,1.7, \mathrm{NSO}_{2} \mathrm{Ph} H\right), 7.65-7.67\left(1 \mathrm{H}, \mathrm{m}, \mathrm{NSO}_{2} \mathrm{Ph} H\right)$, $7.97\left(2 \mathrm{H}, \mathrm{d}, J\right.$ 8.6, $\left.\mathrm{NSO}_{2} \mathrm{Ph} H\right) ;{ }^{13} \mathrm{C}\left\{{ }^{1} \mathrm{H}\right\} \mathrm{NMR}\left(101 \mathrm{MHz}, \mathrm{CDCl}_{3}\right) \delta_{\mathrm{C}}$ : 13.9, 19.8, 44.4, 51.3, 61.7, 116.5, 126.2, 126.6, 128.2, 128.5, 128.6, 128.7, 129.0, 129.5, 130.9, 133.0, 134.2, 136.6, 137.0, 139.2, 141.3, 170.1, 172.2; HRMS (ESI+): $\mathrm{C}_{27} \mathrm{H}_{26} \mathrm{NO}_{5} \mathrm{~S}[\mathrm{M}+\mathrm{H}]^{+}$found 476.1520, requires $476.1526(-1.3 \mathrm{ppm})$.

(3S,4S)-Ethyl 3-([1,1'-biphenyl]-4-yl)-2-oxo-6-phenyl-1(phenylsulfonyl)-1,2,3,4-tetrahydropyridine-4-carboxylate 26

Following general procedure $\mathrm{B}$, the reaction of 4-biphenylacetic acid (84.8 $\mathrm{mg}, 0.4 \mathrm{mmol}), i-\mathrm{Pr}_{2} \mathrm{NEt}(70 \mu \mathrm{L}, 0.4 \mathrm{mmol})$ and pivaloyl chloride $(50 \mu \mathrm{L}, 0.4 \mathrm{mmol})$ in $\mathrm{CH}_{2} \mathrm{Cl}_{2}(1.0 \mathrm{~mL})$, followed by (-)tetramisole $\cdot \mathrm{HCl} 4(9.6 \mathrm{mg}, 0.04 \mathrm{mmol}, 20 \mathrm{~mol} \%)$, ketimine 6 (68.7 $\mathrm{mg}, 0.2 \mathrm{mmol}), i-\mathrm{Pr}_{2} \mathrm{NEt}(35 \mu \mathrm{L}, 0.2 \mathrm{mmol})$ and $\mathrm{CH}_{2} \mathrm{Cl}_{2}(2.0 \mathrm{~mL})$, for $1 \mathrm{~h}$ gave a crude residue of 92:8 dr. Purification by flash chromatography $\left(\mathrm{Et}_{2} \mathrm{O} /\right.$ pet. ether, 1:4) gave 26 (82.2 mg, 0.15 mmol, 76\%, 92:8 dr) as a white solid; $\mathrm{mp} 72-74{ }^{\circ} \mathrm{C} ; v_{\max }$ (ATR): $2978,1730,1489,1448,1369,1170,1112,1087,916 ;[\alpha]_{\mathrm{D}}^{20}+16.2$ (c $0.73, \mathrm{CH}_{2} \mathrm{Cl}_{2}$ ); HPLC (Chiralpak IA, $10 \%$ IPA / hexane, 1.0 $\left.\mathrm{mL} / \mathrm{min}, 254 \mathrm{~nm}, 30{ }^{\circ} \mathrm{C}\right): t_{\mathrm{R}}(3 S, 4 S): 59.4 \mathrm{~min}, t_{\mathrm{R}}(3 R, 4 R): 77.6$ $\min , 97 \%$ ee; ${ }^{1} \mathrm{H}$ NMR $\left(400 \mathrm{MHz}, \mathrm{CDCl}_{3}\right) \delta_{\mathrm{H}}: 1.06(3 \mathrm{H}, \mathrm{t}, J$ 7.1, $\left.\mathrm{OCH}_{2} \mathrm{CH}_{3}\right), 3.79(1 \mathrm{H}, \mathrm{dd}, J$ 9.9, 4.7, C(4)H), $4.03(2 \mathrm{H}, \mathrm{q}, J$ 7.1, $\left.\mathrm{OCH}_{2} \mathrm{CH}_{3}\right), 4.13(1 \mathrm{H}, \mathrm{d}, J 9.9, \mathrm{C}(3) H), 5.89(1 \mathrm{H}, \mathrm{d}, J 4.7, \mathrm{C}(5) H)$, $7.08(2 \mathrm{H}, \mathrm{d}, J$ 8.2, C(3)ArH), 7.27-7.57 (14H, m, $\mathrm{Ar} H$ and $\left.\mathrm{NSO}_{2} \mathrm{Ph} H\right), 7.54-7.66(1 \mathrm{H}, \mathrm{m}, \mathrm{C}(3) p-\mathrm{Ph} H), 7.96(2 \mathrm{H}, \mathrm{dd}, J$ 8.5, 1.0, $\left.\mathrm{NSO}_{2} \mathrm{Ph} H\right) ;{ }^{13} \mathrm{C}\left\{{ }^{1} \mathrm{H}\right\} \mathrm{NMR}\left(101 \mathrm{MHz}, \mathrm{CDCl}_{3}\right) \delta_{\mathrm{C}}: 14.0,45.3,53.9$, 61.8, 116.1, 126.2, 127.2, $127.6(2 \times C), 128.6,128.7,128.9,129.0$, 129.1, 129.5, 134.2, 134.4, 136.6, 139.2, 140.6, 141.1, 141.4, 170.9,
172.1; HRMS (ESI+): $\mathrm{C}_{32} \mathrm{H}_{28} \mathrm{NO}_{5} \mathrm{~S} \quad[\mathrm{M}+\mathrm{H}]^{+}$found 538.1677, requires $538.1683(-1.1 \mathrm{ppm})$.

$(3 S, 4 S)$-Ethyl 3-(naphthalen-2-yl)-2-oxo-6-phenyl-1(phenylsulfonyl)-1,2,3,4-tetrahydropyridine-4-carboxylate 27

Following general procedure $\mathrm{B}$, the reaction of 2-naphthaleneacetic acid $(74.5 \mathrm{mg}, 0.4 \mathrm{mmol}), i-\mathrm{Pr}_{2} \mathrm{NEt}(70 \mu \mathrm{L}, 0.4 \mathrm{mmol})$ and pivaloyl chloride $(50 \mu \mathrm{L}, 0.4 \mathrm{mmol})$ in $\mathrm{CH}_{2} \mathrm{Cl}_{2}(1.0 \mathrm{~mL})$, followed by (-)tetramisole $\cdot \mathrm{HCl} 4(9.6 \mathrm{mg}, 0.04 \mathrm{mmol}, 20 \mathrm{~mol} \%)$, ketimine 6 (68.7 $\mathrm{mg}, 0.2 \mathrm{mmol}), i-\mathrm{Pr}_{2} \mathrm{NEt}(35 \mu \mathrm{L}, 0.2 \mathrm{mmol})$ and $\mathrm{CH}_{2} \mathrm{Cl}_{2}(2.0 \mathrm{~mL})$, for $1 \mathrm{~h}$ gave a crude residue of 91:9 dr. Purification by flash chromatography $\left(\mathrm{Et}_{2} \mathrm{O} /\right.$ pet. ether, 1:4) gave 27 (63.7 mg, 0.12 mmol, $62 \%, 92: 8 \mathrm{dr}$ ) as a white solid; $\mathrm{mp} 178-180{ }^{\circ} \mathrm{C} ; v_{\max }$ (ATR): $2978,1774,1446,1359,1166,1124,1097,1012 ;[\alpha]_{\mathrm{D}}^{20}+17.3(c$ $0.55, \mathrm{CH}_{2} \mathrm{Cl}_{2}$ ); HPLC (Chiralpak AD-H, 30\% IPA / hexane, 1.0 $\left.\mathrm{mL} / \mathrm{min}, 254 \mathrm{~nm}, 30{ }^{\circ} \mathrm{C}\right): t_{\mathrm{R}}(3 S, 4 S): 18.6 \mathrm{~min}, t_{\mathrm{R}}(3 R, 4 R): 30.8$ $\min , 97 \%$ ee; ${ }^{1} \mathrm{H}$ NMR $\left(400 \mathrm{MHz}, \mathrm{CDCl}_{3}\right) \delta_{\mathrm{H}}: 0.95(1 \mathrm{H}, \mathrm{t}, J 7.1$, $\left.\mathrm{OCH}_{2} \mathrm{CH}_{3}\right), 3.86(1 \mathrm{H}, \mathrm{dd}, J 9.9,4.7, \mathrm{C}(4) H), 3.96(2 \mathrm{H}, \mathrm{qd}, J 10.8$, 7.1, $\left.\mathrm{OCH}_{2} \mathrm{CH}_{3}\right), 4.26(1 \mathrm{H}, \mathrm{dd}, J 9.9, \mathrm{C}(3) H), 5.84(1 \mathrm{H}, \mathrm{d}, J 5.2$, $\mathrm{C}(5) H), 7.05(1 \mathrm{H}, \mathrm{dd}, J 8.5,1.9, \mathrm{C}(3) \mathrm{Ar} H), 7.34-7.57(10 \mathrm{H}, \mathrm{m}$, $\mathrm{Ar} H), 7.62-7.83(4 \mathrm{H}, \mathrm{m}, \mathrm{Ar} H), 7.97\left(2 \mathrm{H}, \mathrm{dd}, J 6.8,1.2, \mathrm{NSO}_{2} \mathrm{Ph} H\right)$; ${ }^{13} \mathrm{C}\left\{{ }^{1} \mathrm{H}\right\}$ NMR $\left(101 \mathrm{MHz}, \mathrm{CDCl}_{3}\right) \delta_{\mathrm{C}}: 14.0,45.2,54.3,61.7,116.0$, 126.0, 126.2, 126.4 127.8, 128.2, 128.6, 128.7, 128.7, 128.8, 129.0, 129.6, 132.8, 133.0, 133.3, $134.2(2 \times C), 136.7,139.2,141.4,170.8$, 172.0; HRMS (ESI+): $\mathrm{C}_{30} \mathrm{H}_{26} \mathrm{NO}_{5} \mathrm{~S}[\mathrm{M}+\mathrm{H}]^{+}$found 512.1522, requires $512.1526(-0.8 \mathrm{ppm})$.

\section{$(3 S, 4 S)$-Ethyl 3-(naphthalen-1-yl)-2-oxo-6-phenyl-1-} (phenylsulfonyl)-1,2,3,4-tetrahydropyridine-4-carboxylate 28

Following general procedure $\mathrm{B}$, the reaction of 1-naphthaleneacetic acid $(74.5 \mathrm{mg}, 0.4 \mathrm{mmol}), i-\operatorname{Pr}_{2} \mathrm{NEt}(70 \mu \mathrm{L}, 0.4 \mathrm{mmol})$ and pivaloyl chloride $(50 \mu \mathrm{L}, 0.4 \mathrm{mmol})$ in $\mathrm{CH}_{2} \mathrm{Cl}_{2}(1.0 \mathrm{~mL})$, followed by (-)tetramisole $\cdot \mathrm{HCl} 4(9.6 \mathrm{mg}, 0.04 \mathrm{mmol}, 20 \mathrm{~mol} \%)$, ketimine 6 (68.7 $\mathrm{mg}, 0.2 \mathrm{mmol}), i-\mathrm{Pr}_{2} \mathrm{NEt}(35 \mu \mathrm{L}, 0.2 \mathrm{mmol})$ and $\mathrm{CH}_{2} \mathrm{Cl}_{2}(2.0 \mathrm{~mL})$, for $1 \mathrm{~h}$ gave a crude residue of 91:9 dr. Purification by flash chromatography $\left(\mathrm{Et}_{2} \mathrm{O} /\right.$ pet. ether, 1:4) gave lactam $28(70.8 \mathrm{mg}$, $0.14 \mathrm{mmol}, 69 \%, 92: 8 \mathrm{dr})$ as a white solid; $\mathrm{mp} 176-178{ }^{\circ} \mathrm{C} ; v_{\max }$ (ATR): 2978, 1721, 1446, 1166, 931; $[\alpha]_{\mathrm{D}}^{20}-14.7\left(c 0.58, \mathrm{CH}_{2} \mathrm{Cl}_{2}\right)$; HPLC (Chiralpak IA, 10\% IPA / hexane, $1.0 \mathrm{~mL} / \mathrm{min}, 211 \mathrm{~nm}, 30$ $\left.{ }^{\circ} \mathrm{C}\right): t_{\mathrm{R}}(3 S, 4 S): 30.7 \mathrm{~min}, t_{\mathrm{R}}(3 R, 4 R): 98.1 \mathrm{~min}, 97 \%$ ee; ${ }^{1} \mathrm{H}$ NMR $\left(400 \mathrm{MHz}, \mathrm{CDCl}_{3}\right) \delta_{\mathrm{H}}: 0.95\left(1 \mathrm{H}, \mathrm{t}, J 7.1, \mathrm{OCH}_{2} \mathrm{CH}_{3}\right), 3.89-4.06(3 \mathrm{H}$, $\mathrm{m}, \mathrm{C}(4) \mathrm{H}$ and $\left.\mathrm{OCH}_{2} \mathrm{CH}_{3}\right), 4.91(1 \mathrm{H}, \mathrm{d}, J 8.6, \mathrm{C}(3) H), 5.84(1 \mathrm{H}, \mathrm{d}, J$ 5.2, $\mathrm{C}(5) H), 7.12-7.20(1 \mathrm{H}, \mathrm{m}, \mathrm{C}(3) \mathrm{Ar} H), 7.33-7.36(1 \mathrm{H}, \mathrm{m}$, $\mathrm{C}(3) \mathrm{Ar} H), 7.39-7.50(5 \mathrm{H}, \mathrm{m}, \mathrm{C}(6) \mathrm{Ph} H), 7.50-7.56(2 \mathrm{H}, \mathrm{t}, J$ 7.4, $\left.\mathrm{NSO}_{2} \mathrm{Ph} H\right), 7.66-7.72\left(1 \mathrm{H}, \mathrm{m}, \mathrm{NSO}_{2} \mathrm{Ph} H\right), 7.72-7.76(1 \mathrm{H}, \mathrm{m}$, $\mathrm{C}(3) \operatorname{Ar} H), 7.80(1 \mathrm{H}, \mathrm{d}, J$ 8.3, C(3)ArH), $7.87(1 \mathrm{H}, \mathrm{dd}, J$ 6.8, 2.7, $\mathrm{C}(3) \mathrm{Ar} H), 7.99\left(2 \mathrm{H}, \mathrm{dd}, J 6.8,1.2, \mathrm{NSO}_{2} \mathrm{Ph} H\right) ;{ }^{13} \mathrm{C}\left\{{ }^{1} \mathrm{H}\right\}$ NMR $(126$ $\left.\mathrm{MHz}, \mathrm{CDCl}_{3}\right) \delta_{\mathrm{C}}: 13.8,44.6,51.3,61.7,115.8,123.2,125.2,125.9$, 126.2, 126.7, 126.8, 128.6, 128.6, 128.9, 129.1, 129.3, 129.6, 131.0, 131.7, $134.2(2 \times C), 136.7,139.0,141.4,170.9,171.9$; HRMS (ESI+): $\mathrm{C}_{30} \mathrm{H}_{26} \mathrm{NO}_{5} \mathrm{~S}[\mathrm{M}+\mathrm{H}]^{+}$found 512.1523 , requires 512.1526 $(-0.6 \mathrm{ppm})$.

(3S,4S)-Ethyl 3-(3,4-dimethoxyphenyl)-2-oxo-6-phenyl-1(phenylsulfonyl)-1,2,3,4-tetrahydropyridine-4-carboxylate 29

Following general procedure $\mathrm{B}$, the reaction of 3,4dimethoxyphenylacetic acid (78.4 mg, $0.4 \mathrm{mmol}), i-\mathrm{Pr}_{2} \mathrm{NEt}(70 \mu \mathrm{L}$, $0.4 \mathrm{mmol})$ and pivaloyl chloride $(50 \mu \mathrm{L}, 0.4 \mathrm{mmol})$ in $\mathrm{CH}_{2} \mathrm{Cl}_{2}(1.0$ $\mathrm{mL})$, followed by (-)-tetramisole $\cdot \mathrm{HCl} 4(9.6 \mathrm{mg}, 0.04 \mathrm{mmol}, 20$ mol\%), ketimine 6 ( $68.7 \mathrm{mg}, 0.2 \mathrm{mmol}), i-\mathrm{Pr}_{2} \mathrm{NEt}(35 \mu \mathrm{L}, 0.2 \mathrm{mmol})$ and $\mathrm{CH}_{2} \mathrm{Cl}_{2}(2.0 \mathrm{~mL})$, for $1 \mathrm{~h}$ gave a crude residue of 93:7 dr. Purification by flash chromatography $\left(\mathrm{Et}_{2} \mathrm{O} /\right.$ pet. ether, 2:3) gave 29 
(81.0 mg, $0.16 \mathrm{mmol}, 78 \%$, 97:3 dr) as a white solid; mp 126-128 ${ }^{\circ} \mathrm{C}$; $v_{\max }$ (ATR): 2937, 1782, 1593, 1516, 1448, 1367, 1298, 1224, 1143, 1087, 1026, 979; $[\alpha]_{\mathrm{D}}^{20}+32.6\left(c \quad 0.53, \mathrm{CH}_{2} \mathrm{Cl}_{2}\right)$; HPLC (Chiralpak IA, 30\% IPA / hexane, $\left.0.5 \mathrm{~mL} / \mathrm{min}, 254 \mathrm{~nm}, 30{ }^{\circ} \mathrm{C}\right): t_{\mathrm{R}}$ $(3 S, 4 S): 38.0 \mathrm{~min}, t_{\mathrm{R}}(3 R, 4 R): 53.5 \mathrm{~min}, 98 \%$ ee; ${ }^{1} \mathrm{H}$ NMR $(500$ $\left.\mathrm{MHz}, \mathrm{CDCl}_{3}\right) \delta_{\mathrm{H}}: 1.08\left(3 \mathrm{H}, \mathrm{t}, J 7.1, \mathrm{OCH}_{2} \mathrm{CH}_{3}\right), 3.72(1 \mathrm{H}, \mathrm{dd}, J 9.8$, 4.8, C(4)H), $3.75\left(3 \mathrm{H}, \mathrm{s}, \mathrm{C}(3) \mathrm{ArOCH}_{3}\right), 3.83\left(3 \mathrm{H}, \mathrm{s}, \mathrm{C}(3) \mathrm{ArOCH}_{3}\right)$, 3.94-4.11 $\left(3 \mathrm{H}, \mathrm{m}, \mathrm{C}(3) \mathrm{H}\right.$ and $\left.\mathrm{OCH}_{2} \mathrm{CH}_{3}\right), 5.86(1 \mathrm{H}, \mathrm{d}, J 4.7, \mathrm{C}(5) H)$, $6.50(1 \mathrm{H}, \mathrm{d}, J 1.9, \mathrm{C}(3) \mathrm{Ar} H), 6.58(1 \mathrm{H}, \mathrm{dd}, J 8.2,2.0, \mathrm{C}(3) \mathrm{Ar} H)$, $6.75(1 \mathrm{H}, \mathrm{d}, J$ 8.3, C(3)ArH), 7.35-7.40 (5H, m, C(6)PhH), $7.40(2 \mathrm{H}$, t, $\left.J 7.9, \mathrm{NSO}_{2} \mathrm{Ph} H\right), 7.64\left(1 \mathrm{H}, \mathrm{t}, J 7.5, \mathrm{NSO}_{2} \mathrm{Ph} H\right), 7.95(2 \mathrm{H}, \mathrm{d}, J 7.4$, $\left.\mathrm{NSO}_{2} \mathrm{Ph} H\right) ;{ }^{13} \mathrm{C}\left\{{ }^{1} \mathrm{H}\right\}$ NMR $\left(126 \mathrm{MHz}, \mathrm{CDCl}_{3}\right) \delta_{\mathrm{C}}: 14.1,45.3,53.7$, $56.0(2 \times C), 61.7,111.2,111.6,116.0,121.1,126.1,127.7,128.6$, 128.7, 129.0, 129.5, 134.1, 136.7, 139.3, 141.3, 148.9, 149.0, 170.9, 172.2; HRMS (ESI+): $\mathrm{C}_{28} \mathrm{H}_{31} \mathrm{~N}_{2} \mathrm{O}_{7} \mathrm{~S}\left[\mathrm{M}+\mathrm{NH}_{4}\right]^{+}$found 539.1842, requires $539.1846(-0.8 \mathrm{ppm})$.

(3S,4S)-Ethyl 3-(4-(dimethylamino)phenyl)-2-ox0-6-phenyl-1(phenylsulfonyl)-1,2,3,4-tetrahydropyridine-4-carboxylate 30 Following general procedure $\mathrm{B}$, the reaction of 4(dimethylamino)phenylacetic acid $(71.7 \mathrm{mg}, 0.4 \mathrm{mmol}), i-\mathrm{Pr}_{2} \mathrm{NEt}$ (70 $\mu \mathrm{L}, 0.4 \mathrm{mmol})$ and pivaloyl chloride $(50 \mu \mathrm{L}, 0.4 \mathrm{mmol})$ in $\mathrm{CH}_{2} \mathrm{Cl}_{2}(1.0 \mathrm{~mL})$, followed by $(-)$-tetramisole $\cdot \mathrm{HCl} 4(9.6 \mathrm{mg}, 0.04$ mmol, $20 \mathrm{~mol} \%$ ), ketimine 6 (68.7 mg, $0.2 \mathrm{mmol}), i-\operatorname{Pr}_{2} \mathrm{NEt}(35 \mu \mathrm{L}$, $0.2 \mathrm{mmol})$ and $\mathrm{CH}_{2} \mathrm{Cl}_{2}(2.0 \mathrm{~mL})$, for $1 \mathrm{~h}$ gave a crude residue of $92: 8$ dr. Purification by flash chromatography $\left(\mathrm{Et}_{2} \mathrm{O}\right.$ : pet. ether, $\left.2: 3\right)$ gave 30 (80.3 mg, $0.16 \mathrm{mmol}, 80 \%, 93: 7 \mathrm{dr}$ ) as a white solid; mp 178-180 ${ }^{\circ} \mathrm{C} ; v_{\max }$ (ATR): 2933, 1730, 1516, 1355, 1159, 929; $[\alpha]_{\mathrm{D}}^{20}+30.6(c$ $0.51, \mathrm{CH}_{2} \mathrm{Cl}_{2}$ ); HPLC (Chiralpak IA, 10\% IPA / hexane, 1.0 $\left.\mathrm{mL} / \mathrm{min}, 254 \mathrm{~nm}, 30^{\circ} \mathrm{C}\right): t_{\mathrm{R}}(3 S, 4 S): 30.4 \min , t_{\mathrm{R}}(3 R, 4 R): 55.2$ $\min , 99 \%$ ee; ${ }^{1} \mathrm{H}$ NMR $\left(400 \mathrm{MHz}, \mathrm{CDCl}_{3}\right) \delta_{\mathrm{H}}: 1.09(3 \mathrm{H}, \mathrm{t}, J 7.1$, $\left.\mathrm{OCH}_{2} \mathrm{CH}_{3}\right), 2.90\left(6 \mathrm{H}, \mathrm{s}, \mathrm{N}\left(\mathrm{CH}_{3}\right)_{2}\right), 3.68(1 \mathrm{H}, \mathrm{dd}, J 9.4,5.0, \mathrm{C}(4) H)$, 3.95-4.08 $\left(3 \mathrm{H}, \mathrm{m}, \mathrm{C}(3) \mathrm{H}\right.$ and $\left.\mathrm{OCH}_{2} \mathrm{CH}_{3}\right), 5.85(1 \mathrm{H}, \mathrm{d}, J 5.0, \mathrm{C}(5) H)$, 6.59 (2H, d, $J$ 8.8, C(3)ArH ), 6.85 (2H, d, $J$ 8.7, C(3)ArH), 7.36$7.40(5 \mathrm{H}, \mathrm{m}, \mathrm{C}(6) \mathrm{Ph} H), 7.45-7.53\left(2 \mathrm{H}, \mathrm{m}, \mathrm{NSO}_{2} \mathrm{Ph} H\right), 7.64(1 \mathrm{H}, \mathrm{tt}, J$ 7.1, 1.2, $\left.\mathrm{NSO}_{2} \mathrm{Ph} H\right), 7.94\left(2 \mathrm{H}, \mathrm{dd}, J 8.5,1.2, \mathrm{NSO}_{2} \mathrm{Ph} H\right) ;{ }^{13} \mathrm{C}\left\{{ }^{1} \mathrm{H}\right\}$ NMR $\left(126 \mathrm{MHz}, \mathrm{CDCl}_{3}\right) \delta_{\mathrm{C}}: 14.1,40.6,45.4,53.3,61.6,112.7$, 116.2, 122.7, 126.2, 128.6, 128.6, 128.8, 129.3, 129.5, 134.0, 136.9, 139.4, 141.2, 150.3, 171.1, 172.5; HRMS (ESI+): $\mathrm{C}_{28} \mathrm{H}_{29} \mathrm{~N}_{2} \mathrm{O}_{5} \mathrm{~S}$ $[\mathrm{M}+\mathrm{H}]^{+}$found 505.1786 , requires $505.1792(-1.1 \mathrm{ppm})$.

(3S,4S)-Ethyl 3-(4-methoxyphenyl)-2-oxo-6-phenyl-1(phenylsulfonyl)-1,2,3,4-tetrahydropyridine-4-carboxylate 31

Following general procedure $\mathrm{B}$, the reaction of 4methoxyphenylacetic acid (66.4 mg, $0.4 \mathrm{mmol}), i$ - $\operatorname{Pr}_{2} \mathrm{NEt}(70 \mu \mathrm{L}, 0.4$ mmol) and pivaloyl chloride $(50 \mu \mathrm{L}, 0.4 \mathrm{mmol})$ in $\mathrm{CH}_{2} \mathrm{Cl}_{2}(1.0 \mathrm{~mL})$, followed by (-)-tetramisole $\cdot \mathrm{HCl} 4(9.6 \mathrm{mg}, 0.04 \mathrm{mmol}, 20 \mathrm{~mol} \%)$, ketimine $6(68.7 \mathrm{mg}, 0.2 \mathrm{mmol}), i$ - $\operatorname{Pr}_{2} \mathrm{NEt}(35 \mu \mathrm{L}, 0.2 \mathrm{mmol})$ and $\mathrm{CH}_{2} \mathrm{Cl}_{2}(2.0 \mathrm{~mL})$, for $1 \mathrm{~h}$ gave a crude residue of $92: 8 \mathrm{dr}$. Purification by flash chromatography $\left(\mathrm{Et}_{2} \mathrm{O} /\right.$ pet. ether, $\left.2: 3\right)$ gave 31 (75.5 mg, $0.15 \mathrm{mmol}, 74 \%, 92: 8 \mathrm{dr}$ ) as a white solid; mp 152-154 ${ }^{\circ} \mathrm{C} ; v_{\max }$ (ATR): 2960, 1732, 1716, 1514, 1165, 920; $[\alpha]_{\mathrm{D}}^{20}+28.9(c$ 0.54, $\mathrm{CH}_{2} \mathrm{Cl}_{2}$ ); HPLC (Chiralpak AD-H, 20\% IPA / hexane, 1.0 $\left.\mathrm{mL} / \mathrm{min}, 254 \mathrm{~nm}, 30^{\circ} \mathrm{C}\right): t_{\mathrm{R}}(3 S, 4 S): 25.5 \mathrm{~min}, t_{\mathrm{R}}(3 R, 4 R): 42.8$ $\min , 98 \%$ ee; ${ }^{1} \mathrm{H}$ NMR $\left(500 \mathrm{MHz}, \mathrm{CDCl}_{3}\right) \delta_{\mathrm{H}}: 1.06(3 \mathrm{H}, \mathrm{t}, J 7.1$, $\left.\mathrm{OCH}_{2} \mathrm{CH}_{3}\right), 3.71(1 \mathrm{H}, \mathrm{dd}, J 10.0,4.6, \mathrm{C}(4) H), 3.76(3 \mathrm{H}, \mathrm{s}$, $\left.\mathrm{C}(3) \mathrm{ArOCH}_{3}\right), 3.97-4.06\left(3 \mathrm{H}, \mathrm{m}, \mathrm{C}(3) \mathrm{H}\right.$ and $\left.\mathrm{OCH}_{2} \mathrm{CH}_{3}\right), 5.86(1 \mathrm{H}$, $\mathrm{d}, J$ 4.6, C(5)H), 6.76-6.84 (2H, m, C(3)ArH ), 6.88-6.96 (2H, m, $\mathrm{C}(3) \mathrm{Ar} H), \quad 7.31-7.43 \quad(5 \mathrm{H}, \mathrm{m}, \mathrm{C}(6) \mathrm{Ph} H), \quad 7.46-7.54 \quad(2 \mathrm{H}, \mathrm{m}$, $\left.\mathrm{NSO}_{2} \mathrm{Ph} H\right), 7.65\left(1 \mathrm{H}, \mathrm{m}, \mathrm{NSO}_{2} \mathrm{Ph} H\right), 7.90-7.97\left(2 \mathrm{H}, \mathrm{m}, \mathrm{NSO}_{2} \mathrm{Ph} H\right)$; ${ }^{13} \mathrm{C}\left\{{ }^{1} \mathrm{H}\right\}$ NMR $\left(126 \mathrm{MHz}, \mathrm{CDCl}_{3}\right) \delta_{\mathrm{C}}: 14.1,45.4,53.6,53.5,61.7$,
$114.2,116.2,126.2,127.3,128.6,128.6,128.9,129.5,129.8,134.1$, $136.7, \quad 139.2, \quad 141.2, \quad 159.4,171.0,172.3 ;$ HRMS (ESI+): $\mathrm{C}_{27} \mathrm{H}_{26} \mathrm{NO}_{6} \mathrm{~S}[\mathrm{M}+\mathrm{H}]^{+}$found 492.1473, requires $492.1475(-0.5$ ppm).

(3S,4S)-Ethyl 3-(4-chlorophenyl)-2-oxo-6-phenyl-1(phenylsulfonyl)-1,2,3,4-tetrahydropyridine-4-carboxylate 32

Following general procedure $\mathrm{B}$, the reaction of 4-chlorophenylacetic acid $(68.2 \mathrm{mg}, 0.4 \mathrm{mmol}), i-\mathrm{Pr}_{2} \mathrm{NEt}(70 \mu \mathrm{L}, 0.4 \mathrm{mmol})$ and pivaloyl chloride $(50 \mu \mathrm{L}, 0.4 \mathrm{mmol})$ in $\mathrm{CH}_{2} \mathrm{Cl}_{2}(1.0 \mathrm{~mL})$, followed by (-)tetramisole $\cdot \mathrm{HCl} 4(9.6 \mathrm{mg}, 0.04 \mathrm{mmol}, 20 \mathrm{~mol} \%)$, ketimine 6 (68.7 $\mathrm{mg}, 0.2 \mathrm{mmol}), i-\mathrm{Pr}_{2} \mathrm{NEt}(35 \mu \mathrm{L}, 0.2 \mathrm{mmol})$ and $\mathrm{CH}_{2} \mathrm{Cl}_{2}(2.0 \mathrm{~mL})$, for $2 \mathrm{~h}$ gave a crude residue of 92:8 dr. Purification by flash chromatography $\left(\mathrm{Et}_{2} \mathrm{O} /\right.$ pet. ether, $\left.1: 4\right)$ gave $32(53.5 \mathrm{mg}, 0.11$ mmol, $54 \%, 94: 6 \mathrm{dr}$ ) as a white solid; $m p 136-138^{\circ} \mathrm{C} ; v_{\max }$ (ATR): $2978,1724,1683,1448,1367,1168,1087,921 ;[\alpha]_{\mathrm{D}}^{20}+33.3(c$ $0.55, \mathrm{CH}_{2} \mathrm{Cl}_{2}$ ); HPLC (Chiralpak IA, 30\% IPA / hexane, 0.5 $\left.\mathrm{mL} / \mathrm{min}, 254 \mathrm{~nm}, 30{ }^{\circ} \mathrm{C}\right): t_{\mathrm{R}}(3 S, 4 S): 26.6 \mathrm{~min}, t_{\mathrm{R}}(3 R, 4 R): 42.0$ $\min , 95 \%$ ee; ${ }^{1} \mathrm{H}$ NMR $\left(400 \mathrm{MHz}, \mathrm{CDCl}_{3}\right) \delta_{\mathrm{H}}: 1.06(1 \mathrm{H}, \mathrm{t}, J 7.1$, $\left.\mathrm{OCH}_{2} \mathrm{CH}_{3}\right), 3.72(1 \mathrm{H}, \mathrm{dd}, J 10.5,4.2, \mathrm{C}(4) H), 3.94-4.09(3 \mathrm{H}, \mathrm{m}$, $\mathrm{C}(3) \mathrm{H}$ and $\left.\mathrm{OCH}_{2} \mathrm{CH}_{3}\right), 5.86(1 \mathrm{H}, \mathrm{d}, J 4.5, \mathrm{C}(5) \mathrm{H}), 6.93(2 \mathrm{H}, \mathrm{d}, J$ 8.4, C(3)ArH), 7.25 (2H, d, $J$ 8.5, C(3)ArH), 7.35-7.39 (5H, m, $\mathrm{C}(6) \mathrm{Ph} H), 7.46-7.54\left(2 \mathrm{H}, \mathrm{m}, \mathrm{NSO}_{2} \mathrm{Ph} H\right), 7.66(1 \mathrm{H}, \mathrm{tt}, J$ 7.1, 1.2, $\left.\mathrm{NSO}_{2} \mathrm{Ph} H\right), 7.93\left(2 \mathrm{H}, \mathrm{dd}, J 8.5,1.2, \mathrm{NSO}_{2} \mathrm{Ph} H\right) ;{ }^{13} \mathrm{C}\left\{{ }^{1} \mathrm{H}\right\} \mathrm{NMR}(126$ $\left.\mathrm{MHz}, \mathrm{CDCl}_{3}\right) \delta_{\mathrm{C}}: 14.0,45.1,53.6,61.9,116.0,126.2,128.7,128.7$, $129.1,129.1,129.5,130.2,133.9,134.2,134.3,136.4,139.1,141.4$, 170.7, 171.8; HRMS (ESI+): $\mathrm{C}_{26} \mathrm{H}_{23} \mathrm{~N}^{35} \mathrm{ClO}_{5} \mathrm{~S} \quad[\mathrm{M}+\mathrm{H}]^{+}$found 496.0981, requires 496.0980 ( $+0.2 \mathrm{ppm})$.

$(3 S, 4 S)$-Ethyl 3-(4-bromophenyl)-2-oxo-6-phenyl-1(phenylsulfonyl)-1,2,3,4-tetrahydropyridine-4-carboxylate 33

Following general procedure $\mathrm{B}$, the reaction of 4-bromophenylacetic acid $(86.0 \mathrm{mg}, 0.4 \mathrm{mmol}), i-\mathrm{Pr}_{2} \mathrm{NEt}(70 \mu \mathrm{L}, 0.4 \mathrm{mmol})$ and pivaloyl chloride $(50 \mu \mathrm{L}, 0.4 \mathrm{mmol})$ in $\mathrm{CH}_{2} \mathrm{Cl}_{2}(1.0 \mathrm{~mL})$, followed by (-)tetramisole $\cdot \mathrm{HCl} 4(9.6 \mathrm{mg}, 0.04 \mathrm{mmol}, 20 \mathrm{~mol} \%)$, ketimine 6 (68.7 $\mathrm{mg}, 0.2 \mathrm{mmol}), i-\mathrm{Pr}_{2} \mathrm{NEt}(35 \mu \mathrm{L}, 0.2 \mathrm{mmol})$ and $\mathrm{CH}_{2} \mathrm{Cl}_{2}(2.0 \mathrm{~mL})$, for $2 \mathrm{~h}$ gave a crude residue of 92:8 dr. Purification by flash chromatography $\left(\mathrm{Et}_{2} \mathrm{O} /\right.$ pet. ether, $\left.1: 4\right)$ gave 33 (64.6 mg, 0.13 mmol, $60 \%, 94: 6 \mathrm{dr}$ ) as a white solid; $\mathrm{mp} 140-142{ }^{\circ} \mathrm{C}$; $v_{\max }$ (ATR): 2976, 1726, 1710, 1448, 1168, 925; $[\alpha]_{\mathrm{D}}^{20}+31.1\left(c 0.54, \mathrm{CH}_{2} \mathrm{Cl}_{2}\right)$; HPLC (Chiralpak IA, 30\% IPA / hexane, $0.5 \mathrm{~mL} / \mathrm{min}, 254 \mathrm{~nm}, 30$ $\left.{ }^{\circ} \mathrm{C}\right): t_{\mathrm{R}}(3 S, 4 S): 30.4 \mathrm{~min}, t_{\mathrm{R}}(3 R, 4 R): 45.8 \mathrm{~min}, 95 \%$ ee; ${ }^{1} \mathrm{H}$ NMR $\left(400 \mathrm{MHz}, \mathrm{CDCl}_{3}\right) \delta_{\mathrm{H}}: 1.07\left(1 \mathrm{H}, \mathrm{t}, J 7.1, \mathrm{OCH}_{2} \mathrm{CH}_{3}\right), 3.72(1 \mathrm{H}, \mathrm{dd}, J$ $10.5,4.2, \mathrm{C}(4) H), 3.94-4.09\left(3 \mathrm{H}, \mathrm{m}, \mathrm{C}(3) \mathrm{H}\right.$ and $\left.\mathrm{OCH}_{2} \mathrm{CH}_{3}\right), 5.85$ $(1 \mathrm{H}, \mathrm{d}, J 4.5, \mathrm{C}(5) H), 6.87(2 \mathrm{H}, \mathrm{d}, J$ 8.4, C(3)ArH), 7.32-7.44 (7H, $\mathrm{m}, \mathrm{C}(3) \mathrm{Ar} H$ and $\mathrm{C}(6) \mathrm{Ph} H), 7.46-7.55\left(2 \mathrm{H}, \mathrm{m}, \mathrm{NSO}_{2} \mathrm{Ph} H\right), 7.66(1 \mathrm{H}$, tt, $J$ 7.1, 1.2, $\left.\mathrm{NSO}_{2} \mathrm{Ph} H\right), 7.93\left(2 \mathrm{H}, \mathrm{d}, J 8.5, \mathrm{NSO}_{2} \mathrm{Ph} H\right) ;{ }^{13} \mathrm{C}\left\{{ }^{1} \mathrm{H}\right\}$ NMR $\left(101 \mathrm{MHz}, \mathrm{CDCl}_{3}\right) \delta_{\mathrm{C}}: 14.0,45.1,53.6,61.9,115.9,122.3$, 126.2, 128.7, 128.7, 129.1, 129.5, 130.5, 132.0, 134.3, 134.4, 136.4, 139.1, 141.4, 170.6, 171.7; HRMS (ESI+): $\mathrm{C}_{26} \mathrm{H}_{23} \mathrm{~N}^{79} \mathrm{BrO}_{5} \mathrm{~S}[\mathrm{M}+\mathrm{H}]^{+}$ found 540.0473 , requires $540.0475(-0.3 \mathrm{ppm})$.

$(3 S, 4 S)$-Ethyl 2-oxo-6-phenyl-1-(phenylsulfonyl)-3-(thiophen-3yl)-1,2,3,4-tetrahydropyridine-4-carboxylate 34

Following general procedure $\mathrm{B}$, the reaction of 3-thiophenylacetic acid $(56.9 \mathrm{mg}, 0.4 \mathrm{mmol}), i-\mathrm{Pr}_{2} \mathrm{NEt}(70 \mu \mathrm{L}, 0.4 \mathrm{mmol})$ and pivaloyl chloride $(50 \mu \mathrm{L}, 0.4 \mathrm{mmol})$ in $\mathrm{CH}_{2} \mathrm{Cl}_{2}(1.0 \mathrm{~mL})$, followed by (-)tetramisole $\cdot \mathrm{HCl} 4(9.6 \mathrm{mg}, 0.04 \mathrm{mmol}, 20 \mathrm{~mol} \%)$, ketimine 6 (68.7 $\mathrm{mg}, 0.2 \mathrm{mmol}), i-\mathrm{Pr}_{2} \mathrm{NEt}(35 \mu \mathrm{L}, 0.2 \mathrm{mmol})$ and $\mathrm{CH}_{2} \mathrm{Cl}_{2}(2.0 \mathrm{~mL})$, for $1 \mathrm{~h}$ gave a crude residue of 85:15 dr. Purification by flash chromatography $\left(\mathrm{Et}_{2} \mathrm{O} /\right.$ pet. ether, $\left.1: 4\right)$ gave $34(59.7 \mathrm{mg}, 0.13$ mmol, $64 \%, 92: 8 \mathrm{dr}$ ) as a white solid; $\mathrm{mp} 112-114{ }^{\circ} \mathrm{C}$; $v_{\max }$ (ATR): 
2924, 1728, 1448, 1357, 1303, 1219, 1165, 1120, 1083, 1056, 1029, 1001, 842; $[\alpha]_{\mathrm{D}}^{20}+29.3\left(c 0.59, \mathrm{CH}_{2} \mathrm{Cl}_{2}\right)$; HPLC (Chiralpak IA, 20\% IPA / hexane, $\left.0.5 \mathrm{~mL} / \mathrm{min}, 254 \mathrm{~nm}, 30^{\circ} \mathrm{C}\right): t_{\mathrm{R}}(3 S, 4 S): 40.3 \mathrm{~min}, t_{\mathrm{R}}$ $(3 R, 4 R): 72.1 \mathrm{~min}, 97 \%$ ee; ${ }^{1} \mathrm{H}$ NMR $\left(400 \mathrm{MHz}, \mathrm{CDCl}_{3}\right) \delta_{\mathrm{H}}: 1.14$ $\left(3 \mathrm{H}, \mathrm{t}, J 7.1, \mathrm{OCH}_{2} \mathrm{CH}_{3}\right), 3.68(1 \mathrm{H}, \mathrm{dd}, J 8.1,5.5, \mathrm{C}(4) H), 4.08(2 \mathrm{H}$, qd, $\left.J 7.4,3.6, \mathrm{OCH}_{2} \mathrm{CH}_{3}\right), 4.24(1 \mathrm{H}, \mathrm{dd}, J 8.1, \mathrm{C}(3) H), 5.85(1 \mathrm{H}, \mathrm{d}, J$ 5.5, C(5)H), $6.77(1 \mathrm{H}, \mathrm{dd}, J 5.0,1.3, \mathrm{C}(3) \mathrm{Ar} H), 7.03(1 \mathrm{H}, \mathrm{ddd}, J 2.9$, 1.3, 0.6, C(3)ArH), 7.23-7.26 (1H, m, C(3)ArH), 7.34-7.37 (5H, m, $\mathrm{C}(6) \mathrm{Ph} H), 7.43-7.54\left(2 \mathrm{H}, \mathrm{m}, \mathrm{NSO}_{2} \mathrm{Ph} H\right), 7.64(1 \mathrm{H}, \mathrm{tt}, J$ 7.4, 1.2, $\left.\mathrm{NSO}_{2} \mathrm{Ph} H\right), 7.92\left(2 \mathrm{H}, \mathrm{dd}, J 8.5,1.2, \mathrm{NSO}_{2} \mathrm{Ph} H\right) ;{ }^{13} \mathrm{C}\left\{{ }^{1} \mathrm{H}\right\} \mathrm{NMR}(126$ $\left.\mathrm{MHz}, \mathrm{CDCl}_{3}\right) \delta_{\mathrm{C}}: 14.1,44.6,49.2,61.8,115.3,123.6,126.1,126.5$, 126.9, 128.6, 128.6, 128.9, 129.4, 134.1, 134.9, 136.6, 139.1, 141.4, 170.7, 171.0; HRMS (ESI+): $\mathrm{C}_{24} \mathrm{H}_{22} \mathrm{NO}_{5} \mathrm{~S}_{2}[\mathrm{M}+\mathrm{H}]^{+}$found 468.0933, requires $468.0934(-0.2 \mathrm{ppm})$.

(3R,4S)-Ethyl 2-oxo-6-phenyl-1-(phenylsulfonyl)-3-((E)-prop-1en-1-yl)-1,2,3,4-tetrahydropyridine-4-carboxylate 35

Following general procedure $\mathrm{B}$, the reaction of $(E)$-pent-3-enoic acid $(82 \mu \mathrm{L}, 0.8 \mathrm{mmol}), i$ - $\operatorname{Pr}_{2} \mathrm{NEt}(140 \mu \mathrm{L}, 0.8 \mathrm{mmol})$ and pivaloyl chloride $(50 \mu \mathrm{L}, 0.4 \mathrm{mmol})$ in $\mathrm{CH}_{2} \mathrm{Cl}_{2}(2.0 \mathrm{~mL})$, followed by (-)tetramisole $\cdot \mathrm{HCl} 4(19.2 \mathrm{mg}, 0.08 \mathrm{mmol}, 20 \mathrm{~mol} \%)$, ketimine 6 (137.2 mg, $0.4 \mathrm{mmol}), i-\mathrm{Pr}_{2} \mathrm{NEt}(70 \mu \mathrm{L}, 0.4 \mathrm{mmol})$ and $\mathrm{CH}_{2} \mathrm{Cl}_{2}(4.0$ $\mathrm{mL}$ ), for $1 \mathrm{~h}$ gave a crude residue of 92:8 dr. Purification by flash chromatography (EtOAc / pet. ether, 1:9) gave 35 (89.5 mg, 0.21 mmol, $53 \%, 88: 12 \mathrm{dr}$ ) as a white solid; $m p 56-58{ }^{\circ} \mathrm{C} ; v_{\max }$ (ATR): 2976, 1732, 1448, 1367, 1170, 1085, 1026, 926; $[\alpha]_{\mathrm{D}}^{20}+67.0(c$ 0.53, $\mathrm{CH}_{2} \mathrm{Cl}_{2}$ ); HPLC (Chiralpak OD-H, 5\% IPA / hexane, 1.0 $\left.\mathrm{mL} / \mathrm{min}, 2211 \mathrm{~nm}, 40{ }^{\circ} \mathrm{C}\right): t_{\mathrm{R}}(3 S, 4 R): 15.8 \mathrm{~min}, t_{\mathrm{R}}(3 R, 4 S): 19.5$ $\min , 98 \%$ ee; ${ }^{1} \mathrm{H}$ NMR $\left(400 \mathrm{MHz}, \mathrm{CDCl}_{3}\right) \delta_{\mathrm{H}}: 1.25(3 \mathrm{H}, \mathrm{t}, J$ 7.1, $\left.\mathrm{OCH}_{2} \mathrm{CH}_{3}\right), 1.66\left(3 \mathrm{H}, \mathrm{dd}, J 6.3,1.4, \mathrm{C}(3) \mathrm{CHCHCH}_{3}\right), 3.37(1 \mathrm{H}, \mathrm{dd}$, $J$ 8.4, 5.4, C(4)H), $3.47(1 \mathrm{H}, \mathrm{t}, J 8.2, \mathrm{C}(3) H), 4.15(2 \mathrm{H}, \mathrm{qd}, J 7.1$, 1.4, $\left.\mathrm{OCH}_{2} \mathrm{CH}_{3}\right), 5.30\left(1 \mathrm{H}\right.$, ddq, $\left.J 15.1,8.1,1.5, \mathrm{C}(3) \mathrm{CHCHCH}_{3}\right)$, $5.64\left(1 \mathrm{H}, \mathrm{m}, \mathrm{C}(3) \mathrm{CHCHCH}_{3}\right), 5.80(1 \mathrm{H}, \mathrm{d}, J 5.4, \mathrm{C}(5) H), 7.30-7.38$ $(5 \mathrm{H}, \mathrm{m}, \mathrm{C}(6) \mathrm{Ph} H), 7.44-7.51\left(2 \mathrm{H}, \mathrm{m}, \mathrm{NSO}_{2} \mathrm{Ph} H\right), 7.62(1 \mathrm{H}, \mathrm{tt}, J 7.0$, 1.2, $\left.\mathrm{NSO}_{2} \mathrm{Ph} H\right), 7.90\left(2 \mathrm{H}, \mathrm{dt}, J 8.6,1.5, \mathrm{NSO}_{2} \mathrm{Ph} H\right) ;{ }^{13} \mathrm{C}\left\{{ }^{1} \mathrm{H}\right\} \mathrm{NMR}$ $\left(101 \mathrm{MHz}, \mathrm{CDCl}_{3}\right) \delta_{\mathrm{C}}: 14.3,18.2,43.7,51.5,61.7,115.7,123.8$, $126.1,128.5,128.6,128.8,129.4,132.1,134.1,136.9,139.2,141.2$, 170.9, 171.8; HRMS (ESI+): $\mathrm{C}_{23} \mathrm{H}_{24} \mathrm{NO}_{5} \mathrm{~S}[\mathrm{M}+\mathrm{H}]^{+}$found 426.1368, requires $426.1370(-0.4 \mathrm{ppm})$.

$(3 S, 4 S)$-Ethyl 6-(4-methoxyphenyl)-2-oxo-3-phenyl-1-tosyl1,2,3,4-tetrahydropyridine-4-carboxylate 36

Following general procedure $\mathrm{B}$, the reaction of phenylacetic acid ( 55 $\mathrm{mg}, 0.4 \mathrm{mmol}), i$ - $\mathrm{Pr}_{2} \mathrm{NEt}(70 \mu \mathrm{L}, 0.4 \mathrm{mmol})$ and pivaloyl chloride (49 $\mu \mathrm{L}, 0.4 \mathrm{mmol})$ in $\mathrm{CH}_{2} \mathrm{Cl}_{2}(1.0 \mathrm{~mL})$, followed by (-)tetramisole $\cdot \mathrm{HCl} 4(9.6 \mathrm{mg}, 0.04 \mathrm{mmol}, 20 \mathrm{~mol} \%)$, ketimine 17 (77 $\mathrm{mg}, 0.2 \mathrm{mmol}), i$ - $\mathrm{Pr}_{2} \mathrm{NEt}(35 \mu \mathrm{L}, 0.2 \mathrm{mmol})$ and $\mathrm{CH}_{2} \mathrm{Cl}_{2}(2.0 \mathrm{~mL})$, for $24 \mathrm{~h}$ gave a crude residue of 90:10 dr. Purification by flash chromatography $\left(\mathrm{Et}_{2} \mathrm{O} /\right.$ pet. ether, 40:60) gave 36 (59 mg, 0.12 mmol, 58\%, 90:10 dr) as a white solid; $\mathrm{mp} 132-134{ }^{\circ} \mathrm{C}$; $v_{\max }$ (ATR): 2929, 1718, 1606, 1501, 1361, 1161, 926; $[\alpha]_{\mathrm{D}}^{20}+32.5$ (c 0.52 , $\mathrm{CH}_{2} \mathrm{Cl}_{2}$ ); HPLC (Chiralpak IA, 30\% IPA / hexane, $1.0 \mathrm{~mL} / \mathrm{min}, 254$ $\left.\mathrm{nm}, 30{ }^{\circ} \mathrm{C}\right): t_{\mathrm{R}}(3 S, 4 S): 14.1 \mathrm{~min}, t_{\mathrm{R}}(3 R, 4 R): 22.4 \mathrm{~min}, 99 \%$ ee; ${ }^{1} \mathrm{H}$ NMR (500 MHz, $\left.\mathrm{CD}_{2} \mathrm{Cl}_{2}\right) \delta_{\mathrm{H}}: 1.04\left(3 \mathrm{H}, \mathrm{t}, J 7.0, \mathrm{OCH}_{2} \mathrm{CH}_{3}\right), 2.47$ $\left(3 \mathrm{H}, \mathrm{s}, \mathrm{NSO}_{2} \mathrm{ArCH}_{3}\right), 3.67(1 \mathrm{H}, \mathrm{dd}, J 9.7,4.9, \mathrm{C}(4) H), 3.84(3 \mathrm{H}, \mathrm{s}$, $\left.\mathrm{C}(6) \mathrm{ArOCH}_{3}\right), 3.99\left(2 \mathrm{H}, \mathrm{qd}, J\right.$ 7.2, 2.8, $\left.\mathrm{OCH}_{2} \mathrm{CH}_{3}\right), 4.05(1 \mathrm{H}, \mathrm{d}, J$ 9.7, C(3)H), $5.78(1 \mathrm{H}, \mathrm{d}, J 4.8, \mathrm{C}(5) H), 6.89-6.95$ (4H, m, C(3) $\mathrm{Ph} H$ and $\mathrm{C}(6) \mathrm{Ar} H), 7.22-7.28(3 \mathrm{H}, \mathrm{m}, \mathrm{C}(3) \mathrm{Ph} H), 7.32-7.40(4 \mathrm{H}, \mathrm{m}$, $\mathrm{NSO}_{2} \mathrm{Ar} H$ and $\left.\mathrm{C}(6) \mathrm{Ar} H\right)$, 7.77-7.82 (2H, m, NSO $\left.2 \mathrm{Ar} H\right) ;{ }^{13} \mathrm{C}\left\{{ }^{1} \mathrm{H}\right\}$
NMR $\left(125 \mathrm{MHz}, \mathrm{CD}_{2} \mathrm{Cl}_{2}\right) \delta_{\mathrm{C}}: 14.2,22.0,45.4,54.3,55.9,62.0$, $114.3,114.7,127.7(\times 2), 128.4,129.1,129.7,129.9,130.1,136.2$, $136.8, \quad 141.4, \quad 146.1, \quad 160.6, \quad 171.2, \quad 172.4 ; \quad$ HRMS (ESI+): $\mathrm{C}_{28} \mathrm{H}_{28} \mathrm{NO}_{6} \mathrm{~S}[\mathrm{M}+\mathrm{H}]^{+}$found 506.1625, requires $506.1632(-1.4$ ppm).

$(3 S, 4 S)$-Ethyl

3-(4-(dimethylamino)phenyl)-6-(4methoxyphenyl)-2-oxo-1-tosyl-1,2,3,4-tetrahydropyridine-4carboxylate 37

Following general procedure $\mathrm{B}$, the reaction of 4(dimethylamino)phenylacetic acid $(71.7 \mathrm{mg}, 0.4 \mathrm{mmol}), i-\mathrm{Pr}_{2} \mathrm{NEt}$ (70 $\mu \mathrm{L}, 0.4 \mathrm{mmol})$ and pivaloyl chloride $(50 \mu \mathrm{L}, 0.4 \mathrm{mmol})$ in $\mathrm{CH}_{2} \mathrm{Cl}_{2}(1.0 \mathrm{~mL})$, followed by (-)-tetramisole $\mathrm{HCl} 4(9.6 \mathrm{mg}, 0.04$ mmol, $20 \mathrm{~mol} \%)$, ketimine 17 (77.5 mg, $0.2 \mathrm{mmol}), i$ - $\operatorname{Pr}_{2} \mathrm{NEt}(35$ $\mu \mathrm{L}, 0.2 \mathrm{mmol})$ and $\mathrm{CH}_{2} \mathrm{Cl}_{2}(2.0 \mathrm{~mL})$, for $1 \mathrm{~h}$ gave a crude residue of 95:5 dr. Purification by flash chromatography $\left(\mathrm{Et}_{2} \mathrm{O} /\right.$ pet. ether, 1:4) gave $37(52.0 \mathrm{mg}, 0.095 \mathrm{mmol}, 47 \%, 88: 12 \mathrm{dr})$ as a white solid; $\mathrm{mp}$ $144-146^{\circ} \mathrm{C} ; v_{\max }$ (ATR): $2926,1728,1610,1512,1361,1247,1165$, 1087, 1027, 812; $[\alpha]_{\mathrm{D}}^{20}+23.8\left(c 0.55, \mathrm{CH}_{2} \mathrm{Cl}_{2}\right)$; HPLC (Chiralpak IA, $30 \%$ IPA / hexane, $\left.1.0 \mathrm{~mL} / \mathrm{min}, 254 \mathrm{~nm}, 30^{\circ} \mathrm{C}\right): t_{\mathrm{R}}(3 S, 4 S): 16.7$ $\min , t_{\mathrm{R}}(3 R, 4 R): 19.9 \mathrm{~min}, 91 \%$ ee; ${ }^{1} \mathrm{H} \mathrm{NMR}\left(500 \mathrm{MHz}, \mathrm{CD}_{2} \mathrm{Cl}_{2}\right)$ $\delta_{\mathrm{H}}: 1.09\left(3 \mathrm{H}, \mathrm{t}, J 7.1, \mathrm{OCH}_{2} \mathrm{CH}_{3}\right), 2.48\left(3 \mathrm{H}, \mathrm{s}, \mathrm{NSO}_{2} \mathrm{ArCH}_{3}\right), 2.90$ $\left(6 \mathrm{H}, \mathrm{s}, \mathrm{C}(3) \mathrm{ArN}\left(\mathrm{CH}_{3}\right)_{2}\right), 3.61(1 \mathrm{H}, \mathrm{dd}, J 9.5,5.0, \mathrm{C}(4) H), 3.85(3 \mathrm{H}$, s, C(6)ArOCH$\left.H_{3}\right), 3.93(1 \mathrm{H}, \mathrm{d}, J 9.5, \mathrm{C}(3) H), 4.02(2 \mathrm{H}, \mathrm{qd}, J 7.1,3.9$, $\left.\mathrm{OCH}_{2} \mathrm{CH}_{3}\right), 5.77(1 \mathrm{H}, \mathrm{d}, J 5.0, \mathrm{C}(5) H), 6.58(2 \mathrm{H}, \mathrm{d}, J 8.8, \mathrm{C}(3) \mathrm{Ar} H)$, $6.76(2 \mathrm{H}, \mathrm{d}, J$ 8.7, C(3)ArH), 6.83-6.99 (2H, m, C(6)ArH), 7.26-7.44 $\left(4 \mathrm{H}, \mathrm{m}, \mathrm{NSO}_{2} \mathrm{Ar} H\right.$ and $\left.\mathrm{C}(6) \mathrm{Ar} H\right), 7.80\left(2 \mathrm{H}, \mathrm{d}, J\right.$ 8.4, $\left.\mathrm{NSO}_{2} \mathrm{Ar} H\right)$; ${ }^{13} \mathrm{C}\left\{{ }^{1} \mathrm{H}\right\}$ NMR $\left(126 \mathrm{MHz}, \mathrm{CD}_{2} \mathrm{Cl}_{2}\right) \delta_{\mathrm{C}}: 14.1,21.8,40.6,45.4,53.3$, $55.7,61.7,112.6,114.1,114.8,123.1,127.5,129.5,129.4,129.5$, 129.6, 130.0, 136.8, 141.0, 145.8, 150.5, 160.4, 171.3, 172.8; HRMS (ESI+): $\mathrm{C}_{30} \mathrm{H}_{33} \mathrm{~N}_{2} \mathrm{O}_{6} \mathrm{~S}[\mathrm{M}+\mathrm{H}]^{+}$found 549.2047, requires 549.2054 $(-1.2 \mathrm{ppm})$.

\section{Derivatisations}

Ethyl 2-oxo-3,6-diphenyl-1,2-dihydropyridine-4-carboxylate $44^{24}$ A solution of dihydropyridinone 8 (46.2 $\mathrm{mg}, 0.1 \mathrm{mmol}, 1$ eq.) and HCOONa (34.0 mg, $0.5 \mathrm{mmol}, 5$ eq.) in DMF $(0.5 \mathrm{~mL})$ was treated with $10 \% \mathrm{Pd} / \mathrm{C}(12 \mathrm{~mol} \%)$ and stirred at $60{ }^{\circ} \mathrm{C}$ for $24 \mathrm{~h}$. The reaction was allowed to cool to rt, diluted with water $(5 \mathrm{~mL})$, filtered through Celite $^{\circledR}$, and extracted with EtOAc $(3 \times 5 \mathrm{~mL})$. The combined organic layers were dried over $\mathrm{Na}_{2} \mathrm{SO}_{4}$, filtered, and concentrated in vacuo. The residue was purified by flash column chromatography $\left(\mathrm{CH}_{2} \mathrm{Cl}_{2} / \mathrm{MeOH}, 19: 1\right)$ to give $44(27.6 \mathrm{mg}, 0.086 \mathrm{mmol}, 86 \%)$ as a white solid; mp 182-184 ${ }^{\circ} \mathrm{C}\left\{\right.$ Lit. $^{24} 214{ }^{\circ} \mathrm{C}($ EtOAc $\left.)\right\} ;{ }^{1} \mathrm{H}$ NMR $(500$ $\left.\mathrm{MHz}, \mathrm{CDCl}_{3}\right) \delta_{\mathrm{H}}: 0.95\left(3 \mathrm{H}, \mathrm{t}, J 7.1, \mathrm{OCH}_{2} \mathrm{CH}_{3}\right), 4.07(2 \mathrm{H}, \mathrm{q}, J 7.1$, $\left.\mathrm{OCH}_{2} \mathrm{CH}_{3}\right), 6.77(1 \mathrm{H}, \mathrm{s}, \mathrm{C}(5) H), 7.37-7.41(7 \mathrm{H}, \mathrm{m}, \mathrm{PhH}), 7.46(2 \mathrm{H}$, d, $J 6.9, \mathrm{C}(6) \mathrm{Ph} H), 7.78(2 \mathrm{H}, \mathrm{d}, J 7.7 \mathrm{C}(6) \mathrm{Ph} H), 12.17(1 \mathrm{H}, \mathrm{br}$, $\mathrm{N} H) ;{ }^{13} \mathrm{C}\left\{{ }^{1} \mathrm{H}\right\}$ NMR $\left(126 \mathrm{MHz}, \mathrm{CD}_{2} \mathrm{Cl}_{2}\right) \delta_{\mathrm{C}}: 13.7,62.0,104.7$, 126.7, 128.1, 128.2, 129.4, 129.6, 130.7, 132.4, 134.4, 143.5, 146.0, $163.9,165.7,167.5$. Data consistent with literature. ${ }^{24}$

\section{Ethyl 3,6-diphenyl-2-(tosyloxy)isonicotinate 45}

A stirred solution of pyridone $44(31.9 \mathrm{mg}, 0.1 \mathrm{mmol}, 1 \mathrm{eq}$.$) in$ anhydrous THF $(1 \mathrm{~mL})$ at $-78{ }^{\circ} \mathrm{C}$ under an argon atmosphere was treated with $\mathrm{NaH}$ ( $60 \%$ wt., $6.0 \mathrm{mg}, 0.15 \mathrm{mmol}, 1.5$ eq.) followed by a solution of tosyl chloride (28.6 $\mathrm{mg}, 0.15 \mathrm{mmol}, 1.5$ eq.) in anhydrous THF $(0.5 \mathrm{~mL})$. After $1 \mathrm{~h}$, the reaction mixture was allowed to warm to rt, then heated to $60{ }^{\circ} \mathrm{C}$ for $3 \mathrm{~h}$. The reaction was poured into ice water $(10 \mathrm{~mL})$, neutralised with $\mathrm{K}_{2} \mathrm{CO}_{3}$, and extracted with $\mathrm{CHCl}_{3}(3 \times 10 \mathrm{~mL})$. The combined organics were 
dried over $\mathrm{Na}_{2} \mathrm{SO}_{4}$, filtered, and concentrated in vacuo. The crude was purified by flash chromatography $\left(\mathrm{Et}_{2} \mathrm{O} /\right.$ pet. ether, $\left.3: 7\right)$ to give pyridine 45 (44.6 mg, $0.094 \mathrm{mmol}, 94 \%$ ) as a white solid; mp 122$134{ }^{\circ} \mathrm{C}$; $v_{\max }$ (ATR): 2924, 1732, 1597, 1539, 1375, 1327, 1247, $1192,1178,1168,1155,1024,968 ;{ }^{1} \mathrm{H}$ NMR $\left(500 \mathrm{MHz}, \mathrm{CDCl}_{3}\right) \delta_{\mathrm{H}}$ : $0.94\left(3 \mathrm{H}, \mathrm{t}, J 7.1, \mathrm{OCH}_{2} \mathrm{CH}_{3}\right), 2.47\left(3 \mathrm{H}, \mathrm{s}, \mathrm{OTsCH}_{3}\right), 4.07(2 \mathrm{H}, \mathrm{q}, J$ 7.1, $\left.\mathrm{OCH}_{2} \mathrm{CH}_{3}\right), 7.29(2 \mathrm{H}, \mathrm{d}, J$ 8.1, OTs $H), 7.31-7.35(2 \mathrm{H}, \mathrm{m}, \mathrm{Ph} H)$, 7.37-7.49 (6H, m, $\mathrm{Ph} H), 7.75-7.85(4 \mathrm{H}, \mathrm{m}, \mathrm{Ts} H$ and $\mathrm{Ph} H), 7.99(1 \mathrm{H}$, s, $\mathrm{C}(5) H) ;{ }^{13} \mathrm{C}\left\{{ }^{1} \mathrm{H}\right\}$ NMR $\left(126 \mathrm{MHz}, \mathrm{CDCl}_{3}\right) \delta_{\mathrm{C}}: 13.6,21.9,62.0$, 118.1, 126.3, 127.1, 128.3, 128.4, 128.7, 128.7, 128.9, 129.6, 129.6, 130.1, 133.3, 135.1, 136.6, 144.4, 144.9, 155.0, 155.3, 166.5; HRMS (ESI+): $\mathrm{C}_{27} \mathrm{H}_{24} \mathrm{NO}_{5} \mathrm{~S}[\mathrm{M}+\mathrm{H}]^{+}$found 474.1364 , requires 474.1370 $(-1.2 \mathrm{ppm})$.

(3S,4S)-Ethyl 2-oxo-3,6-diphenyl-1,2,3,4-tetrahydropyridine-4carboxylate 48

A solution of dihydropyridinone $8(92.3 \mathrm{mg}, 0.2 \mathrm{mmol}, 1 \mathrm{eq}$.$) in$ anhydrous THF $(2 \mathrm{~mL})$ at $-78{ }^{\circ} \mathrm{C}$ under an argon atmosphere was treated dropwise with a pre-formed solution of sodium $(18.4 \mathrm{mg}, 0.8$ mmol, 4 eq.) and naphthalene (102.5 mg, $0.8 \mathrm{mmol}, 4$ eq.) in anhydrous THF $(2 \mathrm{~mL})$ that had been stirred for $1.5 \mathrm{~h}$ at $\mathrm{rt}$. The reaction was monitored by TLC and when complete $(1 \mathrm{~h})$, quenched with brine $(5 \mathrm{~mL})$ and extracted with EtOAc $(3 \times 5 \mathrm{~mL})$. The combined organics were dried over $\mathrm{MgSO}_{4}$, filtered, and the solvent removed in vacuo to obtain the crude product ( $>99: 1 \mathrm{dr})$. Purification by flash chromatography $\left(\mathrm{Et}_{2} \mathrm{O} /\right.$ pet. ether, $\left.1: 1\right)$ to give 48 (29.5 mg, $0.092 \mathrm{mmol}, 46 \%,>99: 1 \mathrm{dr}$ ) as a white solid; mp 100$102{ }^{\circ} \mathrm{C}$; $v_{\max }$ (ATR): 3246, 2964, 1728, 1670, 1651, 1456, 1265, 1155; $[\alpha]_{\mathrm{D}}^{20}+136.5\left(c 0.26, \mathrm{CH}_{2} \mathrm{Cl}_{2}\right)$; HPLC (Chiralpak OD-H, $30 \%$ IPA / hexane, $\left.0.5 \mathrm{~mL} / \mathrm{min}, 270 \mathrm{~nm}, 30{ }^{\circ} \mathrm{C}\right): t_{\mathrm{R}}(3 S, 4 S): 36.4 \mathrm{~min}, t_{\mathrm{R}}$ (3R, 4R): $29.2 \mathrm{~min}, 99 \%$ ee; ${ }^{1} \mathrm{H}$ NMR $\left(400 \mathrm{MHz}, \mathrm{CDCl}_{3}\right) \delta_{\mathrm{H}}: 1.18$ $\left(3 \mathrm{H}, J 7.1, \mathrm{OCH}_{2} \mathrm{CH}_{3}\right), 3.76(1 \mathrm{H}, \mathrm{dd}, J 7.5,4.8, \mathrm{C}(4) H), 4.13(2 \mathrm{H}, \mathrm{q}$, $J$ 7.1, $\left.\mathrm{OCH}_{2} \mathrm{CH}_{3}\right), 4.19(1 \mathrm{H}, \mathrm{d}, J 7.5, \mathrm{C}(3) H), 5.43(1 \mathrm{H}, \mathrm{dd}, J 4.8$, 1.5, $\mathrm{C}(5) H), 7.29-7.35(5 \mathrm{H}, \mathrm{m}, \mathrm{C}(3) \mathrm{Ph} H), 7.40-7.43 \quad(2 \mathrm{H}, \mathrm{m}$, $\mathrm{C}(6) \mathrm{Ph} H)$, 7.45-7.47 (2H, m, C(6)PhH); ${ }^{13} \mathrm{C}\left\{{ }^{1} \mathrm{H}\right\} \mathrm{NMR}(101 \mathrm{MHz}$, $\left.\mathrm{CDCl}_{3}\right) \delta_{\mathrm{C}}: 14.2,46.7,48.5,61.5,99.3,125.3,127.8,128.4,128.9$, 129.2, 129.6, 134.5, 137.3, 138.2, 170.8, 171.9; HRMS (ESI+): $\mathrm{C}_{20} \mathrm{H}_{20} \mathrm{NO}_{3}[\mathrm{M}+\mathrm{H}]^{+}$found 322.1443 , requires 322.1438 (+1.6 ppm). (3S,4S)-Ethyl 5-bromo-2-oxo-3,6-diphenyl-1-(phenylsulfonyl)1,2,3,4-tetrahydropyridine-4-carboxylate 49

A stirred solution of dihydropyridinone 8 ( $46.2 \mathrm{mg}, 0.1 \mathrm{mmol}, 1$ eq.) in anhydrous $\mathrm{CH}_{2} \mathrm{Cl}_{2}(10 \mathrm{~mL})$ at $-78{ }^{\circ} \mathrm{C}$ under an argon atmosphere was treated dropwise with bromine $(12.8 \mu \mathrm{L}, 0.25 \mathrm{mmol}, 2.5$ eq.) dropwise. $i$ - $\operatorname{Pr}_{2} \mathrm{NEt}(19.0 \mu \mathrm{L}, 0.11 \mathrm{mmol}, 1.1$ eq.) was added and the reaction mixture allowed to warm to rt. The reaction was monitored by TLC and complete in $2 \mathrm{~h}$. The reaction mixture was quenched with saturated aqueous $\mathrm{Na}_{2} \mathrm{~S}_{2} \mathrm{O}_{3}(10 \mathrm{~mL})$, the layers separated and the aqueous extracted with $\mathrm{CH}_{2} \mathrm{Cl}_{2}(3 \times 10 \mathrm{~mL})$. The combined organic layers were washed with brine $(10 \mathrm{~mL})$, dried over $\mathrm{MgSO}_{4}$, filtered, and concentrated in vacuo. The crude product was purified by flash chromatography $\left(\mathrm{Et}_{2} \mathrm{O} /\right.$ pet. ether, 1:4) to give 49 (46.3 mg, $0.086 \mathrm{mmol}, 86 \%,>99: 1 \mathrm{dr})$ as a white solid; mp $130-132{ }^{\circ} \mathrm{C} ; v_{\max }$ (ATR): 2924, 1750, 1732, 1446, 1371, 1181, 1066, 885; $[\alpha]_{\mathrm{D}}^{20}$ -26.7 ( c 0.61, $\mathrm{CH}_{2} \mathrm{Cl}_{2}$ ); HPLC (Chiralpak IA, 30\% IPA / hexane, 1.0 $\left.\mathrm{mL} / \mathrm{min}, 254 \mathrm{~nm}, 30{ }^{\circ} \mathrm{C}\right): t_{\mathrm{R}}(3 S, 4 S): 10.9 \mathrm{~min}, t_{\mathrm{R}}(3 R, 4 R): 15.3$ $\min , 97 \%$ ee; ${ }^{1} \mathrm{H}$ NMR $\left(500 \mathrm{MHz}, \mathrm{CDCl}_{3}\right) \delta_{\mathrm{H}}: 1.36(3 \mathrm{H}, \mathrm{t}, J 7.1$, $\left.\mathrm{OCH}_{2} \mathrm{CH}_{3}\right), 4.00(1 \mathrm{H}, \mathrm{d}, J 3.4, \mathrm{C}(4) H), 4.33(2 \mathrm{H}, \mathrm{ddq}, J 47.0,10.8$, 7.1, $\left.\mathrm{OCH}_{2} \mathrm{CH}_{3}\right), 4.54(1 \mathrm{H}, \mathrm{d}, J 3.4, \mathrm{C}(3) H), 7.19(2 \mathrm{H}, \mathrm{d}, J 7.5$, $\mathrm{C}(3) \mathrm{Ph} H), 7.23-7.39(10 \mathrm{H}, \mathrm{m}, \mathrm{Ph} H), 7.55\left(1 \mathrm{H}, \mathrm{t}, J 7.4, \mathrm{NSO}_{2} \mathrm{Ph} H\right)$, $7.59\left(2 \mathrm{H}, \mathrm{t}, J 7.4, \mathrm{NSO}_{2} \mathrm{Ph} H\right) ;{ }^{13} \mathrm{C}\left\{{ }^{1} \mathrm{H}\right\} \mathrm{NMR}\left(126 \mathrm{MHz}, \mathrm{CDCl}_{3}\right) \delta_{\mathrm{C}}$ :
$14.2,53.8,54.4,62.8,110.2,127.6,127.9,128.4,128.6,129.0$, 129.1, 129.3, 129.8, 133.9, 134.2, 134.9, 137.1, 139.0, 168.9, 170.4; HRMS (ESI+): $\mathrm{C}_{26} \mathrm{H}_{23} \mathrm{~N}^{79} \mathrm{BrO}_{5} \mathrm{~S}[\mathrm{M}+\mathrm{H}]^{+}$found 540.0469, requires $540.0475(-1.1 \mathrm{ppm})$.

(2S,3S)-1-Ethyl 4-methyl 2-(2-oxo-2-phenylethyl)-3phenylsuccinate 50

A solution of dihydropyridinone 8 (46.2 $\mathrm{mg}, 0.1 \mathrm{mmol}, 1$ eq.) and magnesium turnings $(24.3 \mathrm{mg}, 1 \mathrm{mmol}, 10$ eq.) in $\mathrm{MeOH}(15 \mathrm{~mL})$ was stirred at rt overnight. The reaction was quenched with $1 \mathrm{M} \mathrm{HCl}$ $(15 \mathrm{~mL})$ and extracted with $\mathrm{CH}_{2} \mathrm{Cl}_{2}(3 \times 15 \mathrm{~mL})$. The combined organic layers were dried over anhydrous $\mathrm{Na}_{2} \mathrm{SO}_{4}$, filtered, and concentrated in vacuo. The crude product was purified by flash chromatography $\left(\mathrm{Et}_{2} \mathrm{O} /\right.$ pet. ether, 1:9) to give $\mathbf{5 0}$ (12.4 mg, 0.035 mmol, $35 \%,>99: 1 \mathrm{dr}$ ) as a white solid; $\mathrm{mp} 80-82{ }^{\circ} \mathrm{C} ; v_{\max }$ (ATR): $2916,1718,1681,1448,1398,1336,1271,1251,1153,1002 ;[\alpha]_{\mathrm{D}}^{20}$ $+79.7\left(c\right.$ 0.47, $\left.\mathrm{CH}_{2} \mathrm{Cl}_{2}\right)$; HPLC (Chiralpak IA, 30\% IPA / hexane, 0.5 $\left.\mathrm{mL} / \mathrm{min}, 254 \mathrm{~nm}, 30{ }^{\circ} \mathrm{C}\right): t_{\mathrm{R}}(3 S, 4 S): 13.6 \mathrm{~min}, t_{\mathrm{R}}(3 R, 4 R): 16.7$ $\min , 97 \%$ ee; ${ }^{1} \mathrm{H}$ NMR $\left(500 \mathrm{MHz}, \mathrm{CDCl}_{3}\right) \delta_{\mathrm{H}}: 0.88(3 \mathrm{H}, \mathrm{t}, J$ 7.1, $\left.\mathrm{OCH}_{2} \mathrm{CH}_{3}\right), 3.17\left(1 \mathrm{H}, \mathrm{dd}, J 17.3,3.3, \mathrm{C}(4) H^{4} \mathrm{H}^{\mathrm{B}}\right), 3.58(1 \mathrm{H}, \mathrm{dd}, J$ 17.3, 10.3, C(4) $\left.\mathrm{H}^{\mathrm{A}} H^{B}\right), 3.67-3.77\left(4 \mathrm{H}, \mathrm{m}, \mathrm{C}(3) H\right.$ and $\left.\mathrm{OCH}_{3}\right), 3.85$ $\left(2 \mathrm{H}, \mathrm{m}, \mathrm{OCH}_{2} \mathrm{CH}_{3}\right), 3.92(1 \mathrm{H}, \mathrm{d}, J 9.5, \mathrm{C}(2) H), 7.26-7.34(5 \mathrm{H}, \mathrm{m}$, $\mathrm{Ph} H), 7.45$ (2H, dd, $J$ 8.3, 7.2, $\mathrm{Ph} H)$, 7.52-7.59 (1H, m, $\mathrm{Ph} H)$, 7.90$7.97(2 \mathrm{H}, \mathrm{m}, \mathrm{Ph} H) ;{ }^{13} \mathrm{C}\left\{{ }^{1} \mathrm{H}\right\} \mathrm{NMR}\left(126 \mathrm{MHz}, \mathrm{CDCl}_{3}\right) \delta_{\mathrm{C}}: 13.8,38.4$, 44.8, 52.5, 53.5, 60.8, 128.0, 128.2, 128.7, $128.8(2 \times C), 133.4$, 135.8, 136.5, 172.6, 173.2, 197.7; HRMS (ESI+): $\mathrm{C}_{21} \mathrm{H}_{23} \mathrm{O}_{5}[\mathrm{M}+\mathrm{H}]^{+}$ found 355.1542, requires 355.1540 ( $+0.6 \mathrm{ppm})$. Pyridone 44 (19.2 $\mathrm{mg}, 0.06 \mathrm{mmol}, 60 \%)$ was also isolated from this reaction.

$N$-((3S,4S)-5-hydroxy-3-(hydroxymethyl)-1,4-diphenylpent-1-en1-yl)benzenesulfonamide 51

A solution of dihydropyridinone 8 (46.2 $\mathrm{mg}, 0.1 \mathrm{mmol}, 1$ eq.) in anhydrous THF $(1 \mathrm{~mL})$ at $0{ }^{\circ} \mathrm{C}$ under an argon atmosphere was treated dropwise with $2.0 \mathrm{M} \mathrm{LiAlH}_{4}$ in THF $(0.1 \mathrm{~mL}, 0.2 \mathrm{mmol}, 2$ eq.). The reaction was stirred at $0{ }^{\circ} \mathrm{C}$ for $1 \mathrm{~h}$ and quenched with 0.1 $\mathrm{M} \mathrm{HCl}(5 \mathrm{~mL})$. The reaction mixture was extracted with $\mathrm{Et}_{2} \mathrm{O}(3 \times 5$ $\mathrm{mL}$ ), the combined organic layers dried over $\mathrm{MgSO}_{4}$, filtered, and concentrated in vacuo. The crude product was purified by flash chromatography $\left(\mathrm{Et}_{2} \mathrm{O} /\right.$ pet. ether, 1:19) to give $\mathbf{5 1}(27.6 \mathrm{mg}, 0.065$ mmol, $65 \%,>99: 1 \mathrm{dr}$ ) as a white solid; $\mathrm{mp} 74-76{ }^{\circ} \mathrm{C} ; v_{\max }$ (ATR): $2953,1887,1490,1448,1330,1170,1112,1039,1010,950 ;[\alpha]_{\mathrm{D}}^{20}$ $-1.1\left(c\right.$ $0.45, \mathrm{CH}_{2} \mathrm{Cl}_{2}$ ); HPLC (Chiralpak AD-H, 30\% IPA / hexane, $\left.1.0 \mathrm{~mL} / \mathrm{min}, 254 \mathrm{~nm}, 30^{\circ} \mathrm{C}\right): t_{\mathrm{R}}(3 S, 4 S): 9.2 \mathrm{~min}, t_{\mathrm{R}}(3 R, 4 R): 11.1$ $\min , 97 \%$ ee; ${ }^{1} \mathrm{H}$ NMR $\left(400 \mathrm{MHz}, \mathrm{CDCl}_{3}\right) \delta_{\mathrm{H}}: 2.65(1 \mathrm{H}, \mathrm{ddd}, J 11.7$, 4.9, 3.1, C $(4) H), 2.67-1.81(1 \mathrm{H}, \mathrm{m}, \mathrm{C}(3) H), 3.18(2 \mathrm{H}, \mathrm{d}, J 5.2$, $\left.\mathrm{C}(3) \mathrm{CH}_{2} \mathrm{OH}\right), 3.60\left(1 \mathrm{H}, \mathrm{dd}, J 11.5,4.7, \mathrm{C}(5) H^{4} \mathrm{H}^{\mathrm{B}}\right), 3.65-3.73(1 \mathrm{H}$, $\left.\mathrm{m}, \mathrm{C}(5) \mathrm{H}^{\mathrm{A}} H^{B}\right), 5.59(1 \mathrm{H}, \mathrm{dd}, J 9.6, \mathrm{C}(2) H), 7.09-7.19(2 \mathrm{H}, \mathrm{m}$, $\mathrm{C}(4) \mathrm{Ph} H), 7.20-7.42(6 \mathrm{H}, \mathrm{m}, \mathrm{Ph} H), 7.41-7.50(4 \mathrm{H}, \mathrm{m}, \mathrm{Ph} H$ and $\left.\mathrm{NSO}_{2} \mathrm{Ph} H\right), 7.50-7.59\left(1 \mathrm{H}, \mathrm{m}, \mathrm{NSO}_{2} \mathrm{Ph} H\right), 7.75(2 \mathrm{H}, \mathrm{dd}, J$ 8.4, 1.3, $\left.\mathrm{NSO}_{2} \mathrm{Ph} H\right), 8.11(1 \mathrm{H}$, br s, $\mathrm{N} H) ;{ }^{13} \mathrm{C}\left\{{ }^{1} \mathrm{H}\right\}$ NMR $\left(126 \mathrm{MHz}, \mathrm{CDCl}_{3}\right)$ $\delta_{\mathrm{C}}: 41.5,48.6,65.0,65.8,125.5,127.4,127.5,127.5,128.2,128.3$, $128.6,128.9,129.0,132.8,137.5,138.2,140.3,140.7$; HRMS (ESI+): $\mathrm{C}_{24} \mathrm{H}_{25} \mathrm{NO}_{4} \mathrm{SNa}[\mathrm{M}+\mathrm{Na}]^{+}$found 446.1389 , requires 446.1397 $(-1.7 \mathrm{ppm})$.

$(1 S, 4 S, 5 R, 6 S)$-Ethyl 3-oxo-1,4-diphenyl-2-(phenylsulfonyl)-7oxa-2-azabicyclo[4.1.0]heptane-5-carboxylate 52

A solution of dihydropyridinone $8(46.2 \mathrm{mg}, 0.1 \mathrm{mmol}, 1$ eq.) in $\mathrm{CH}_{2} \mathrm{Cl}_{2}(1 \mathrm{~mL})$ at $0{ }^{\circ} \mathrm{C}$ was treated with $m$-CPBA (70\% wt., 37.0 $\mathrm{mg}, 0.15 \mathrm{mmol}, 1.5$ eq.). The reaction was allowed to warm to $\mathrm{rt}$ and stirred for $24 \mathrm{~h}$, before being quenched with sat. $\mathrm{NaHCO}_{3}(2 \mathrm{~mL})$. The layers were separated and the aqueous layer extracted with 
$\mathrm{CH}_{2} \mathrm{Cl}_{2}(3 \times 5 \mathrm{~mL})$. The combined organics were dried over $\mathrm{MgSO}_{4}$, filtered, and the solvent removed in vacuo to obtain the crude product ( $>99: 1 \mathrm{dr})$. Purification by flash chromatography $\left(\mathrm{Et}_{2} \mathrm{O} /\right.$ pet. ether, 1:4) gave $52(34.2 \mathrm{mg}, 0.072 \mathrm{mmol}, 72 \%,>99: 1 \mathrm{dr})$ as a white solid; mp 116-118 ${ }^{\circ} \mathrm{C}$; $v_{\max }$ (ATR): 2976, 2360, 2322, 1726, 1448, 1361, 1273, 1174; $[\alpha]_{\mathrm{D}}^{20}-29.8\left(c \quad 0.50, \mathrm{CH}_{2} \mathrm{Cl}_{2}\right) ;{ }^{1} \mathrm{H}$ NMR $(500$ $\left.\mathrm{MHz}, \mathrm{CD}_{2} \mathrm{Cl}_{2}\right) \delta_{\mathrm{H}}: 0.84\left(3 \mathrm{H}, J 7.1, \mathrm{OCH}_{2} \mathrm{CH}_{3}\right), 3.13(1 \mathrm{H}, \mathrm{dd}, J 13.1$, 3.7, C(4)H), $3.79(1 \mathrm{H}, \mathrm{d}, J 3.79, \mathrm{C}(5) H), 3.85(2 \mathrm{H}, \mathrm{q}, J$ 7.1, $\left.\mathrm{OCH}_{2} \mathrm{CH}_{3}\right), 4.02(1 \mathrm{H}, \mathrm{d}, J 13.1, \mathrm{C}(3) H), 6.87(2 \mathrm{H}, \mathrm{dd}, J$ 7.3, 2.0, $\mathrm{C}(3) \mathrm{Ph} H)$, 7.24-7.28 (3H, $\mathrm{m}, \mathrm{C}(3) \mathrm{Ph} H$ and $\mathrm{C}(6) \mathrm{Ph} H)$, 7.42-7.55 $(7 \mathrm{H}, \mathrm{m}, \mathrm{Ph} H), 7.63-7.55\left(1 \mathrm{H}, \mathrm{m}, \mathrm{NSO}_{2} \mathrm{Ph} H\right), 7.85-7.90(2 \mathrm{H}, \mathrm{m}$, $\left.\mathrm{NSO}_{2} \mathrm{Ph} H\right) ;{ }^{13} \mathrm{C}\left\{{ }^{1} \mathrm{H}\right\}$ NMR $\left(126 \mathrm{MHz}, \mathrm{CD}_{2} \mathrm{Cl}_{2}\right) \delta_{\mathrm{C}}: 13.9,49.9,51.1$, $62.2,64.6,70.0,125.9,128.8,129.0,129.2,129.4,129.6,129.9(2 \times$ C), 134.2, 134.8, 136.5, 138.7, 169.9, 170.8; HRMS (ESI+): $\mathrm{C}_{24} \mathrm{H}_{25} \mathrm{NO}_{4} \mathrm{SNa}[\mathrm{M}+\mathrm{Na}]^{+}$found 446.1389 , requires $446.1397(-1.7$ ppm).

\section{Notes and references}

EaStCHEM, School of Chemistry, University of St Andrews, North Haugh, St Andrews, Fife, KY16 9ST, UK. Fax: +44 (0)1334 463808; Tel: +44 (0)1334 463896; E-mail: ads10@st-andrews.ac.uk

$\dagger$ Electronic Supplementary Information (ESI) available: ${ }^{1} \mathrm{H}$ and ${ }^{13} \mathrm{C}$ $\left\{{ }^{1} \mathrm{H}\right\}$ NMR spectra of all novel compounds and HPLC data. See DOI: $10.1039 / \mathrm{b} 000000 \mathrm{x} /$

1 For a review on the organocatalytic activation of carboxylic acids see: L. C. Morrill and A. D. Smith, Chem. Soc. Rev., 2014, 43, 6214.

2 G. S. Cortez, R. L. Tennyson and D. Romo, J. Am. Chem. Soc., 2001, 123, 7945 .

3 (a) G. S. Cortez, S. H. Oh and D. Romo, Synthesis, 2001, 1731; (b) S. H. Oh, G. S. Cortez and D. Romo, J. Org. Chem., 2005, 70, 2835; (c) H. Henry-Riyad, C. Lee, V. C. Purohit and D. Romo, Org. Lett., 2006, 8, 4363; (d) G. Ma, H. Nguyen and D. Romo, Org. Lett., 2007, 9, 2143; (e) V. C. Purohit, A. S. Matla and D. Romo, J. Am. Chem. Soc., 2008, 130, 10478; (f) H. Nguyen, G. Ma and D. Romo, Chem. Commun., 2010, 46, 4803; (g) K. A. Morris, K. M. Arendt, S. H. Oh and D. Romo, Org. Lett., 2010, 12, 3764; (h) C. A. Leverett, V. C. Purohit and D. Romo, Angew. Chem. Int. Ed., 2010, 49, 9479; (i) H. Nguyen, G. Ma, T. Gladysheva, T. Fremgen and D. Romo, J. Org. Chem., 2010, 76, 2; (j) G. Liu and D. Romo, Angew. Chem. Int. Ed., 2011, 50, 7537; (k) G. Liu, M. E. Shirley and D. Romo, J. Org. Chem., 2012, 77, 2496; (1) C. A. Leverett, V. C. Purohit, A. G. Johnson, R. L. Davis, D. J. Tantillo and D. Romo, J. Am. Chem. Soc., 2012, 134, 13348.

4 For a review on isothiourea catalysis see: J. E. Taylor, S. D. Bull, J. M. J. Williams, Chem. Soc. Rev. 2012, 41, 2109.

5 (a) D. Belmessieri, L. C. Morrill, C. Simal, A. M. Z. Slawin and A. D. Smith, J. Am. Chem. Soc., 2011, 133, 2714; (b) D. Belmessieri, A. de la Houpliere, E. D. D. Calder, J. E. Taylor and A. D. Smith, Chem. Eur. J., 2014, 20, 9762.

6 D. Belmessieri, D. B. Cordes, A. M. Z. Slawin and A. D. Smith, Org Lett., 2013, 15, 3472.

7 L. C. Morrill, J. Douglas, T. Lebl, A. M. Z. Slawin, D. J. Fox and A. D. Smith, Chem. Sci., 2013, 4, 4146.
8 S. R. Smith, J. Douglas, H. Prevet, P. Shapland, A. M. Z. Slawin and A. D. Smith, J. Org. Chem., 2014, 79, 1626.

9 C. Simal, T. Lebl, A. M. Z. Slawin and A. D. Smith, Angew. Chem. Int. Ed., 2012, 51, 3653.

10 D. G. Stark, L. C. Morrill, P.-P. Yeh, A. M. Z. Slawin, T. J. C. O'Riordan and A. D. Smith, Angew. Chem. Int. Ed., 2013, 52, 11642.

11 P.-P. Yeh, D. S. B. Daniels, D. B. Cordes, A. M. Z. Slawin and A. D. Smith, Org. Lett., 2014, 16, 964.

12 (a) L. C. Morrill, T. Lebl, A. M. Z. Slawin and A. D. Smith, Chem. Sci., 2012, 3, 2088; (b) E. R. T. Robinson, C. Fallan, C. Simal, A. M. Z. Slawin and A. D. Smith, Chem. Sci., 2013, 4, 2193; (c) L. C. Morrill, L. A. Ledingham, J.-P. Couturier, J. Bickel, A. D. Harper, C. Fallan and A. D. Smith, Org. Biomol. Chem., 2014, 12, 624; (d) L. C. Morrill, S. M. Smith, A. M. Z. Slawin and A. D. Smith, J. Org. Chem., 2014, 79, 1640; (e) S. R. Smith, S. M. Leckie, R. Holmes, J. Douglas, C. Fallan, P. Shapland, D. Pryde, A. M. Z. Slawin and A. D. Smith, Org. Lett., 2014, 16, 2506; (f) L. C. Morrill, D. G. Stark, J. E. Taylor, S. R. Smith, J. A. Squires, A. C. A. D’Hollander, C. Simal, P. Shapland, T. J. C. O'Riordan and A. D. Smith, Org. Biomol. Chem., 2014, 12, 9016.

13 (a) T.-Y. Jian, P.-L. Shao and S. Ye, Chem. Commun., 2011, 47, 2381; (b) T.-Y. Jian, L.-H. Sun and S. Ye, Chem. Commun., 2012, 48, 10907.

14 Reaction of Michael acceptor 9 with enantiopure isothiourea (-)-4 did not improve the diastereoselectivity. For this reason it was not investigated further.

15 D. L. Boger and A. M. Kasper, J. Am. Chem. Soc., 1989, 111, 1517.

16 H.-H. Lu, X.-F. Wang, C.-J. Yao, J.-M. Zhang, H. Wu and W.-J. Xiao, Chem. Commun., 2009, 4251.

17 Ketimines 6 and 17 were purified on a Biotage ${ }^{\circledR}$ Isolera $^{\mathrm{TM}} 4$, using

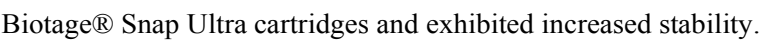

18 CCDC 1034010 contains the supplementary crystallographic data for dihydropyridinone 31. This data can be obtained free of charge from The Cambridge Crystallographic Data Centre via www.ccdc.cam.ac.uk/data_request/cif.

19 (a) Y. Nagao, T. Hirata, S. Goto, S. Sano, A. Kakehi, K. Iizuka and M. Shiro, J. Am. Chem. Soc., 1998, 120, 3104; (b) V. I. Minkin and R. M. Minyaev, Chem. Rev., 2001, 101, 1247; (c) K. A. Brameld, B. Kuhn, D. C. Reuter and M. Stahl, J. Chem. Inf. Model., 2008, 48, 1; (d) P. Liu, X. Yang, V. B. Birman and K. N. Houk, Org. Lett., 2012, 14, 3288 .

20 J. Cheng, Z. Huang and Y. R. Chi, Angew. Chem. Int. Ed., 2013, 52, 8592 .

$21 m$-CPBA oxidation of related dihydropyrones lead to $\gamma$-lactones, see: S. Chen, L. Hao, Y. Zhang, B. Tiwari and Y. R. Chi, Org. Lett., 2013, 15, 5822 .

22 CCDC 1034010 contains the supplementary crystallographic data for epoxide 52. This data can be obtained free of charge from The Cambridge Crystallographic Data Centre via www.ccdc.cam.ac.uk/data_request/cif

23 D. L. Boger and W. L. Corbett, J. Org. Chem., 1992, 57, 4777.

24 S. Perrin, K. Monnier, B. Laude, M. M. Kubicki and O. Blacque, Tetrahedron Lett., 1998, 39, 1753. 
